\title{
A PENALIZED APPROACH TO MIXED MODEL SELECTION VIA CROSS VALIDATION
}

\author{
Jingwei Xiong
}

A Dissertation

Submitted to the Graduate College of Bowling Green

State University in partial fulfillment of

the requirements for the degree of

\section{DOCTOR OF PHILOSOPHY}

December 2017

Committee:

Junfeng Shang, Advisor

Angela Thomas,

Graduate Faculty Representative

Hanfeng Chen

John Chen 
Copyright (C)December 2017

Jingwei Xiong

All rights reserved 
ABSTRACT

Junfeng Shang, Advisor

A linear mixed model is a useful technique to explain observations by regarding them as realizations of random variables, especially when repeated measurements are made to statistical units, such as longitudinal data. However, in practice, there are often too many potential factors considered affecting the observations, while actually, they are not. Therefore, statisticians have been trying to select significant factors out of all the potential factors, where we call the process model selection. Among those approaches for linear mixed model selection, penalized methods have been developed profoundly over the last several decades.

In this dissertation, to solve the overfitting problem in most penalized methods and improve the selection accuracy, we mainly focus on a penalized approach via cross-validation. Unlike the existing methods using the whole data to fit and select models, we split the fitting process and selection into two stages. More specifically, an adaptive lasso penalized function is customized in the first stage and marginal BIC criterion is used in the second stage. We consider that the main advantage of our approach is to reduce the dependency between models construction and evaluation.

Because of the complex structure of mixed models, we adopt a modified Cholesky decomposition to reparameterize the model, which in turn significantly reduces the dimension of the penalized function. Additionally, since random effects are missing, there is no closed form for the maximizer of the penalized function, thus we implement EM algorithm to obtain a full inference of parameters. Furthermore, due to the computation limit and moderately small samples in practice, some noisy factors may still remain in the model, which is particularly obvious for fixed effects. To eliminate the noisy factors, a likelihood ratio test is employed to screen the fixed effects. Regarding the overall process, we call it adaptive lasso via cross-validation.

Additionally, we demonstrate that the proposed approach possesses selection and estimation consistency simultaneously. Moreover, simulation studies and real data examples are both provided to justify the method validity. 
At the very end, a brief conclusion is drawn and some possible further improvements are discussed. 


\section{ACKNOWLEDGMENTS}

A long but amazing journey has come to its end. During this period of growth and learning, many people have offered me valued help and supports.

First and foremost, I would like to thanks my advisor, Dr. Junfeng Shang. Her insights and enthusiasm keep inspiring me to go further in academic research. Whatever difficulties I come across, she is aside to offer assistance. Not only to my study, I have also learned her attitude of perfection to everything in life.

My humble gratitude also goes to every committee member: Dr. Hanfeng Chen, Dr. John Chen, and Dr. Angela Thomas. Thanks for their time and valuable comments on the improvement of my dissertation.

Finally, I would like to appreciate everyone in the Department of Mathematics and Statistics: professors, staff, and classmates. I have been spending a fantastic time with them in daily working and social life. They share my happiness and sorrows, and I will forever remember them. 


\section{TABLE OF CONTENTS}

CHAPTER 1 INTRODUCTION $\ldots \ldots \ldots \ldots \ldots \ldots \ldots$

1.1 Motivation and Objective $\ldots \ldots \ldots \ldots \ldots \ldots$

1.2 Outline. . . . . . . . . . . . . . . . . . . . . . . . 11

CHAPTER 2 LINEAR MODEL SELECTION . . . . . . . . . . . . . . . . . . . . . . . . 13

$2.1 \quad$ Linear Regression Models $\ldots \ldots$. . . . . . . . . . . . . . . . . 13

2.2 Subset Methods with Commonly Used Criteria $\ldots \ldots \ldots$

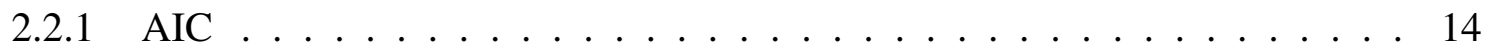

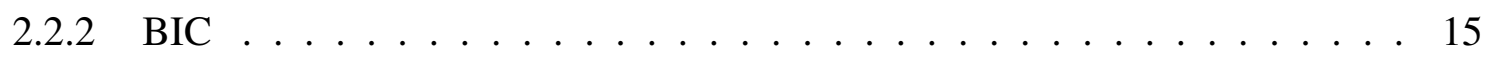

2.2 .3 Subset Methods . . . . . . . . . . . . . . . . . . . . . . . . . . . 15

2.3 Selection Approaches via Penalized Method $\ldots \ldots \ldots$

2.3 .1 Penalized Function . . . . . . . . . . . . . . . . . . . . 16

2.3 .2 Lasso . . . . . . . . . . . . . . . . . . 17

2.3 .3 Least Angle Regression . . . . . . . . . . . . . . . . . . . . . . 18

2.3 .4 Coordinate Descent Algorithm . . . . . . . . . . . . . . . . . . . . . 19

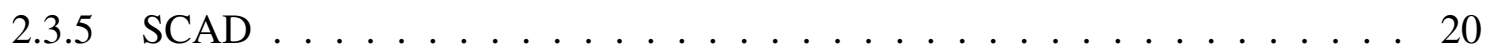

2.3 .6 Adaptive Lasso . . . . . . . . . . . . . . . . . . . . 21

2.3 .7 Tuning Parameter Selection $\ldots \ldots \ldots \ldots \ldots$

CHAPTER 3 LINEAR MIXED MODEL SELECTION $\ldots \ldots \ldots \ldots \ldots$

$3.1 \quad$ Linear Mixed Models $\ldots \ldots \ldots$. . . . . . . . . . . . . . . . . 23

3.2 Commonly Used Criteria in Linear Mixed Models . . . . . . . . . . . . . . . . 24

3.3 Log-Likelihood Function in Linear Mixed Models $\ldots \ldots \ldots$ 
3.4 Cholesky Decomposition . . . . . . . . . . . . . . . . . . . . 26

3.5 Parameter Transformation $\ldots \ldots \ldots \ldots \ldots$

3.6 Selection Approaches via Penalized Method in Linear Mixed Models . . . . . . . . 28

\section{CHAPTER 4 ADAPTIVE LASSO VIA CROSS-VALIDATION FOR LINEAR MIXED}

MODEL SELECTION $\ldots \ldots \ldots \ldots \ldots \ldots \ldots \ldots \ldots \ldots \ldots \ldots$

4.1 Penalized Function $\ldots \ldots \ldots \ldots$

4.2 Tuning Parameter selection via cross-validation $\ldots \ldots \ldots 33$

4.2 .1 Step One . . . . . . . . . . . . . . . . . . . . . . 34

$4.2 .2 \quad$ Step Two $\ldots \ldots \ldots \ldots \ldots \ldots$

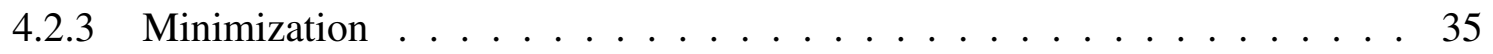

4.2 .4 Univariate Solution for Penalized Quadratic Form . . . . . . . . . . . . 37

4.3 Asymptotic Results . . . . . . . . . . . . . . . . . . . . . . . . . . . . . . . . 39

$4.3 .1 \quad$ Notations and Assumptions $\ldots \ldots \ldots \ldots$. . . . . . . . . 40

4.3 .2 Selection Consistency $\ldots \ldots \ldots \ldots$

4.3 .3 Estimation Consistency $\ldots \ldots \ldots$. . . . . . . . . . . . . 46

4.3 .4 Convergence Rate . . . . . . . . . . . . . . . . . . . . . . . 49

4.4 Computation Procedure $\ldots \ldots \ldots \ldots \ldots$

$4.4 .1 \quad$ Update of Fixed and Diagonal Random Effect Estimates . . . . . . . . . 53

$4.4 .2 \quad$ Update of Non-diagonal Random Effect Estimates $\ldots \ldots \ldots$. . . . . . 55

4.4 .3 Fixed Effects Screening . . . . . . . . . . . . . . . . . . . 60

CHAPTER 5 SIMULATON STUDY $\ldots \ldots \ldots \ldots \ldots$. . . . . . . . . . . . 63

5.1 Presentation of Simulations $\ldots \ldots \ldots$. . . . . . . . . . . . . 63

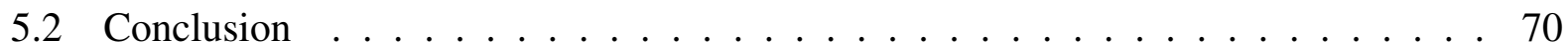

CHAPTER 6 APPLICATION $\ldots \ldots \ldots \ldots \ldots \ldots \ldots \ldots$. . . . . . . . . . . . . 72

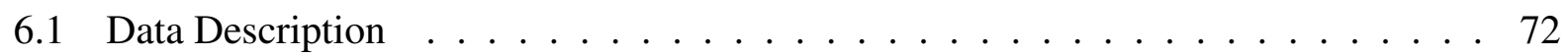

6.2 Analysis in Linear Mixed Models $\ldots \ldots \ldots \ldots$. . . . . . . . . . . 75 
viii

$6.3 \quad$ Implementation and Results $\ldots \ldots \ldots \ldots \ldots \ldots . \ldots . \ldots . \ldots 76$

CHAPTER 7 CONCLUSION AND FURTHER DISCUSSION $\ldots \ldots \ldots \ldots \ldots$

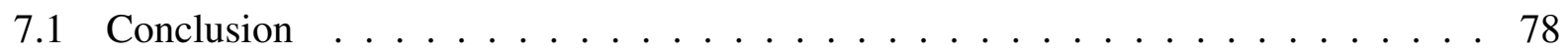

7.2 Further Discussion $\ldots \ldots \ldots \ldots \ldots$

BIBLIOGRAPHY . . . . . . . . . . . . . . . . . . . . . 81

APPENDIX A SELECTED R PROGRAMS . . . . . . . . . . . . . . . . . 86 


\section{LIST OF FIGURES}

Figure

1.1 Fitting with a high degree polynomial function . . . . . . . . . . . . . . 2

1.2 Judging by cross-validation $\ldots \ldots \ldots \ldots \ldots$

4.1 Comparing the difference to existing procedures . . . . . . . . . . . . 34

$6.1 \quad$ Distance of every child. . . . . . . . . . . . . . . . . . 73

6.2 Mean distance, red for males and black for females. . . . . . . . . . . . . . . 73 


\section{LIST OF TABLES}

Table

Page

$1.1 \quad$ Selection results for Null model $\ldots \ldots \ldots \ldots$

1.2 Selection results for Triangle model $\ldots \ldots \ldots \ldots$

$5.1 \quad$ Uniform design matrix with $n=5$. . . . . . . . . . . . . . . . 65

5.2 Uniform design matrix with $n=5 \ldots \ldots$. . . . . . . . . . . . 66

5.3 Uniform design matrix with $n=5$. . . . . . . . . . . . . . . . . . . 67

5.4 Normal design matrix with $n=5 \ldots \ldots \ldots$. . . . . . . . . . . 67

5.5 Uniform design matrix with $n=10 \ldots \ldots$. . . . . . . . . . . . . 68

$5.6 \quad$ Uniform design matrix with $n=10$. . . . . . . . . . . . . . . . . . 68

$5.7 \quad$ Uniform design matrix with $m=60, n=10, q=10 \quad \ldots . \ldots$. . . . . . . . 69

$5.8 \quad$ Uniform design matrix with $n=10 \ldots \ldots$. . . . . . . . . . . 70

6.1 Georges'Study $1 \ldots \ldots \ldots \ldots$. . . . . . . . . . . . . . . . . . . . . . . .

6.2 Georges'Study $2 \ldots \ldots \ldots \ldots \ldots$. . . . . . . . . . . . . . . . . . . . . .

6.3 Marginal criteria for model $(6.1) \ldots \ldots \ldots \ldots$

6.4 Marginal criteria for all models $\ldots \ldots \ldots$. . . . . . . . . . . 76 


\section{CHAPTER 1 INTRODUCTION}

\subsection{Motivation and Objective}

Model selection is known as the process of selecting a statistical model from a potential set of candidate models with best inductive bias. Conceptually, inductive bias is the set of assumptions that the learner uses to predict outputs given inputs that it has not encountered [Mitchell, 1980]. However, in practice, there is no explicit definition of the optimal inductive bias, while it can only be explained as a rough description. Some commonly inductive biases, such as minimum features, minimum description length, and minimum cross-validation error are widely accepted by statisticians to construct a good model. With the regular assumptions, a model is often evaluated from two perspectives: goodness of fit and parsimony. In a general understanding, the goodness of fit is to consider how well the model fits the data and parsimony indicate the modeling complexity. An ideal model is believed to have the simplest structure while capturing the true underlying trend of the data.

Unfortunately, it is tricky that the two objectives above are somehow contradictory. When we are pursuing a better-fitted model to explain all the variations of the data, modeling complexity often increases. Here we take a look at an example provided by Frost [2015], which illustrates the conflicting associations between the two objectives.

In Figure 1.1, the horizontal axis displays the independent variable and the vertical axis displays the response variable. A fitted line constructed by the third order polynomial function is plotted, when the average vertical distances are considered to evaluate the goodness of fit. To improve the goodness of fit, one can always add higher-order terms of the independent variable to bend and twist the fitted line arbitrarily, as close as possible to all the observations, where the realization is guaranteed by Weierstrass Theorem [Stone, 1948]. Nevertheless, some variations might be caused by random errors, and the overestimation of the fluctuations probably makes the fitted line 




Figure 1.1: Fitting with a high degree polynomial function

too complicate for the data. As a result, a good model should balance the goodness of fit and complexity, and the main objective of the model selection is to choose the simplest model which adequately accommodates the data. In this dissertation, we will mainly focus on the discussion of a modeling approach in regression analysis.

Linear regression is one of the most classic modeling techniques to fit the data. It takes a concise expression while the expected values depend linearly on the unknown parameters, which is much easier than a non-linear form. Specifically, in linear regression, a linear predictor function is built to indicate the relationship between explanatory variables and response variables, which minimizes the "lack of fit". Among all the measures of "lack of fit", mean squared error loss is the most popular one to construct the "best" model. However, in practice, the fitting process may result in a model excessively complex in structure, which is different from the true underlying model and gives a poor prediction accuracy. As illustrated in Figure 1.1, if we construct a model with minimum mean squared error loss with respect to the observed sample data, the fitting process is quite possible to conclude a too complicated, higher order model. Nevertheless, this model may fit poorly to the population data. The result of getting an excessively complex model is known as 
the overfitting problem.

In order to avoid misspecification of the underlying model, cross-validation has been accepted as a powerful technique. A typical procedure of cross-validation is to partition the sample data into two parts, namely, a training set and test set. Instead of fitting with the whole dataset, only training set is considered to construct the model, and test set is utilized to compute the "lack of fit". We call the above process one round cross-validation or holdout-validation. Practically, among all the candidate models, the one with minimum "lack of fit" to the test set would be suggested.

To reduce the dependence between estimated "lack of fit" and validation set, it is encouraged to perform multiple rounds to compute the average "lack of fit" from different test sets. The multiple rounds cross-validation is also known as the k-fold cross-validation. It starts with splitting the data into $\mathrm{k}$ subsets, and repeat the holdout-validation $\mathrm{k}$ times. Each time, one subset is taken as the test set while the rest data form the training set. The average "lack of fit" corresponding to the $\mathrm{k}$ test sets can be treated as a better criterion, which indicates how accurately the predictive model performs. Similar to the holdout-validation, the one with minimum "lack of fit" is adopted as the "best" model. Moreover, an extreme case of the k-fold cross-validation is that the number of folds equals the sample size, where we call this procedure leave-one-out cross-validation. Nevertheless, $\mathrm{k}$-fold cross-validation is only recommended when the sample size is large, and bootstrap could be employed to generate a pseudo sample with enough size.

Over the last several decades, numerous studies show that cross-validation with appropriate measures of fit leads to a more accurate estimation of model prediction [Kohavi, 1995, Grossman et al., 2010], which is helpful in capturing the underlying trend of a true model. Furthermore, a comprehensive overview of the example in the following research [Schneider, 1997] is provided to discuss the issue more throughly.

In Figure 1.2, the data in the top two graphs are part of the pseudo data generated by a simple underlying function with significant noises. Two models are constructed to fit the data separately, and their corresponding fitted lines are plotted. From the results, we can see that the first line fluctuates a lot, while the second line is relatively smooth. Regarding candidate model structures, 


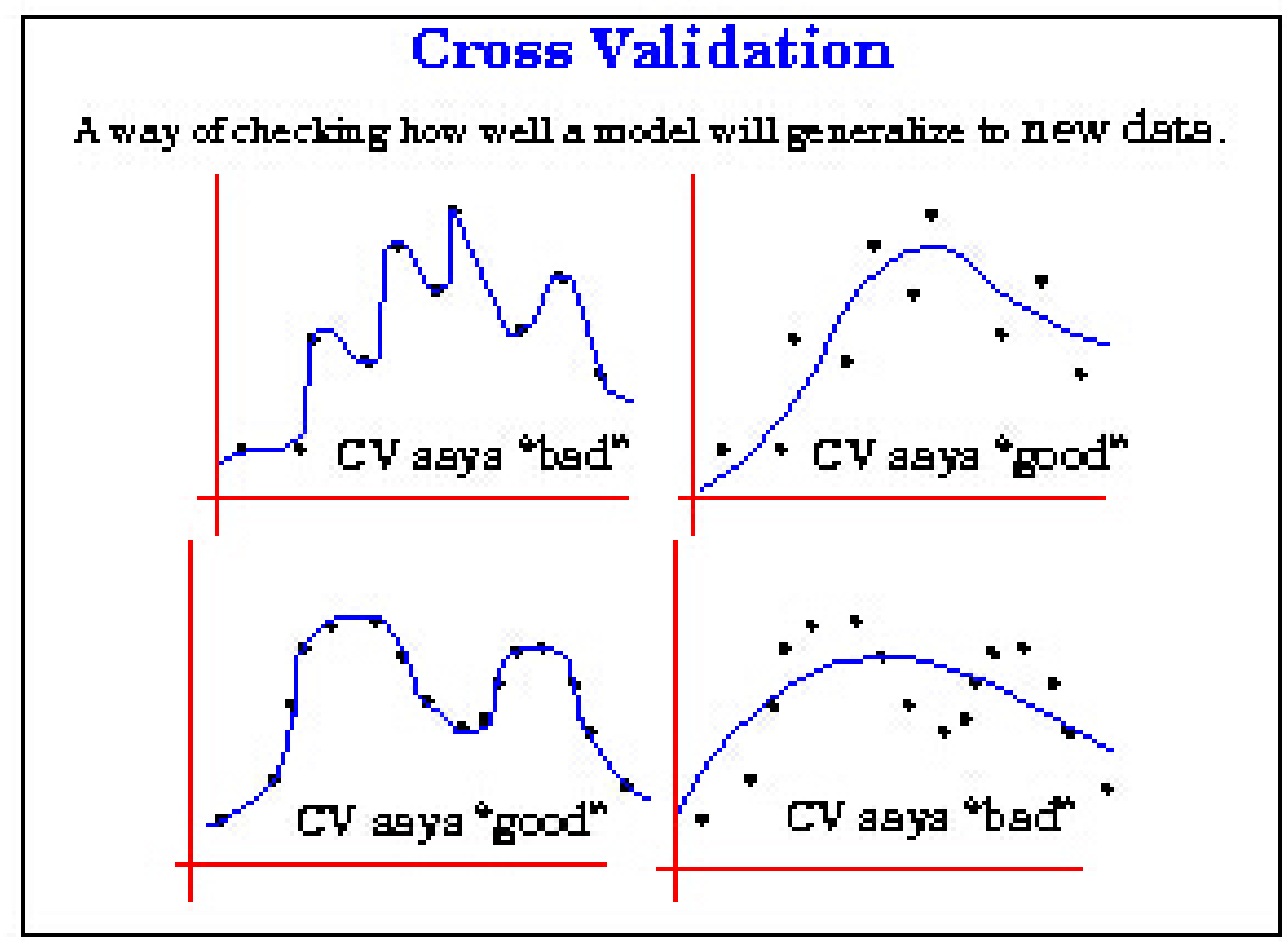

Figure 1.2: Judging by cross-validation

the first fitted line represents a more complicate model while the second fitted line stands for a simpler model. To check the modeling performance, the rest of the pseudo data is used as validation set and cross-validation is implemented for both candidate models. Computation results indicate that the second model has a smaller mean squared error, and consequently, cross-validation favors a simpler form to a complex form, which is compatible with the fact that the true underlying model is simple.

On the contrary, the data in the bottom two graphs are generated from a complex underlying function with no noise. This time, cross-validation shows that the model with very little smoothing is better, which favors a more complex model. Since the underlying model is complex, the result coincides with the fact again.

In addition, not only numerous real data examples in statistic research and machine learning support cross-validation, there is also solid theoretical basis behind it. For instance, to demonstrate the validity of cross-validation from information criteria perspective, leave-one-out crossvalidation is proved asymptotically equivalent as Akaike information criterion [Akaike, 1973] in 
model selection [Stone, 1977].

Akaike information criterion, known as the AIC, was first investigated by Hirotugu Akaike from the idea of minimizing the KullbackLeibler discrepancy. Since then, this criterion has been widely applied in model selection due to its good performance and concise form. However, one of the main drawbacks of AIC is that we only focus on generating the model which most adequately fits the data, regardless of the fact that some models are not even in the set of candidate models. As a result, AIC may cause the inconsistency problem in model selection. The further analysis implies that AIC often tends to select an excessively complex model instead of the true model, which makes the criterion frustrating, as well as leave-one-out cross-validation. To justify the drawbacks, a rigorous proof of the inconsistency of leave-one-out cross-validation was provided by Shao [1993]. However, in this paper, it also points out that when certain conditions are met, especially, the validation data size diverges in the same order as the sample size, cross-validation achieves model selection consistency. For example, in a 2-fold cross-validation, the validation data size is one half of the sample size, which makes the validation data size and sample size diverge in the same order, thus consistency can be achieved. Since the development of AIC, many other criteria have been also proposed, such as Conceptual Predictive Statistic [Mallows, 1973], Bayesian Information Criterion [Schwarz, 1978], Hannan Quinn Criterion [Hannan and Quinn, 1979] etc.. Meanwhile, AIC itself is also developed for being applied in different situations, such as $\mathrm{AIC}_{\mathrm{c}}[$ Hurvich and Tsai, 1989] etc..

With a proper criterion, the goal of model selection turns to be the job of choosing the subset corresponding to the "best" criterion. Practically, forward stepwise and backward stepwise subset methods are often considered as efficient algorithms to select the "best" subset. Nonetheless, the number of potential subsets increases tremendously as the number of predictors increases. When the number of predictors equals $p$, the number of potential subsets is $2^{p}$, which makes the computation very expensive in a high dimensional case. In addition, the fitting process is to add or drop a variables in each step, which makes the process discontinuous. Consequently, the selection result is not stable and sensitive to slight changes of the data. To overcome those 
problems, a continuous process with less computation costs is desired, and penalized approaches have been developed and widely accepted. Instead of considering each subset separately, penalized approaches choose a tuning parameter in a preassigned interval, which continuously shrink some coefficients to zero to achieve the model selection.

In recent several years, penalized approaches have been developed profoundly in model selection since Tibshirani [1996] proposed lasso approach. Unlike traditional subset methods, by continuously shrinking the coefficients towards zero, lasso approach eliminates the noise factors and concludes a simplified model. Moreover, through appropriately choosing the tuning parameter, it can identify the true sparsity and obtain a consistent estimator of the true coefficients simultaneously. However, lasso consistency fails quite often in reality since some assumptions are easily violated. For the sake of ensuring consistency in some mild regularity conditions, a variety of lasso type methods have been developed to extend its application. For example, Zou [2006] analyzed the inconsistency problem of the lasso in some scenarios and proposed the adaptive lasso approach, which successfully extended the application of lasso to a more general setting. In the paper, it proves that adaptive lasso enjoys the oracle property [Fan and Lv, 2001], which means the procedure identifies the true sparse pattern with probability tending to 1 and the coefficient estimates converge to the true values in a certain rate. In addition to lasso and adaptive lasso, many other penalized methods with convex penalties have been discussed as well, such as Dantzig selector [Candes and Tao, 2007]. However, it is not necessary to limit the penalty function with a convex form, and some exceptions are SCAD [Fan and Lv, 2001] or MCP [Zhang, 2010], where the latter two approaches possess non-convex penalties. Considering the computation efficiency, a convex form is generally preferred.

Another important issue for penalized approaches is the selection of tuning parameters. Since the candidate models and coefficient estimates are closely depended on the tuning parameter, its selection is always the key to obtain a good simplified model. Regarding this issue, a typical way is to implement cross-validation to select the "best" tuning parameter. Briefly speaking, we choose the tuning parameter with the candidate model that has minimum mean squared error correspond- 
ing to test data. Moreover, recent studies indicate that the shrinkage problem with lasso penalty is not always the true reason for the over selection phenomenon, while the over selection could be caused by the choice of "lack of fit" in cross-validation. In the regular normal settings, it has been demonstrated that to select the tuning parameters by implementing standard cross-validation with minimum mean squared error as the "lack of fit" cause the overfitting problem [Wasserman and Roeder, 2009]. Therefore, to avoid overfitting, a screening step is suggested for the candidate models to achieve the selection consistency.

To improve the tuning parameter selection, we consider implementing cross-validation to choose the tuning parameter that gives smallest BIC corresponding to the validation set. On the other hand, to make the approach more general, the coefficients are penalized with the adaptive lasso. With cross-validation and adaptive lasso penalty, the procedure gives an estimation of the simplified model, and we name it as adaptive lasso via cross-validation [Xiong and Shang, 2016]. Here, a brief overview and some selected results are presented to illustrate the improvement of adaptive lasso via cross-validation.

Under the regular settings of linear regression models, namely:

$$
y_{i}=X_{i}^{\prime} \beta+\epsilon_{i}, i=1,2, \ldots, n,
$$

where $\left(X_{i}, y_{i}\right)$ are i.i.d observations, and $\epsilon_{i} \sim N\left(0, \sigma^{2}\right)$. In this case, two underlying models are considered.

1. Null model: $\beta=\operatorname{rep}(0,20)$, which contains 20 zero coefficients for the fixed effects.

2. Triangle model: $\beta=(9,8,7, \ldots, 1,0, \ldots, 0)$, which contains 9 nonzero coefficients and 11 zero coefficients for the fixed effects.

Part of the simulation results are summarized in Table 1.1 and Table 1.2, corresponding to the two models above.

In Table 1.1 and 1.2, Lasso.MSE stands for the approach of using lasso penalty with mean squared error to select tuning parameter; A.Lasso.BIC stands for the approach of using adaptive 


\begin{tabular}{|l|l|l|l|}
\hline Null Model & FP & Type I Error & Average Power \\
\hline Lasso.MSE & 0.0023 & 0.0036 & 0 \\
\hline A.Lasso.BIC & 0.0016 & 0.0032 & 0 \\
\hline
\end{tabular}

Table 1.1: Selection results for Null model

\begin{tabular}{|l|l|l|l|}
\hline Tri Model & FP & Type I Error & Average Power \\
\hline Lasso.MSE & 0.0027 & 0.030 & 0.9950 \\
\hline A.Lasso.BIC & 0.0020 & 0.0225 & 0.9972 \\
\hline
\end{tabular}

Table 1.2: Selection results for Triangle model

lasso penalty with BIC to select tuning parameter; and FP represents false positive rate. Meanwhile, both tuning parameter selections are conducted with cross-validation. From the output, we can tell that A.Lasso.BIC approach lower Type I error and false positive rate in both models. In addition, the rejecting power (in the triangle model) of non-zero coefficients is also greater for A.Lasso.BIC, which indicates that we have a higher probability to identify the significant coefficients. Considering the good performance of A.Lasso.BIC in linear regression models, we are motivated to further extend its application to linear mixed models.

A linear mixed model is an extension of a linear regression model, in which the linear predictors contain random effects in addition to the usual fixed effects. It was first mentioned by Henderson et al. [1959] for modeling a wide range of data types, including clustered, longitudinal and spatial data. The main objectives of model selection in the mixed scenario are to choose the significant fixed variables and to detect covariance structure of the random effects. Because of extensively application of linear mixed models, either subset method or penalized method has been extended to mixed settings. Regarding subset methods, proper modifications of the criterion have been made to consider the influence of random effects, such as Marginal AIC [Sugiura, 1978], Conditional 
AIC [Vaida and Blanchard, 2005] and marginal BIC. With these new criteria, we may select the "best" subset from candidate models.

In spite of those extended criteria, computation cost is still a major concern for linear mixed model selection. As analyzed earlier in linear regression models, subset methods are almost impossible to enumerate all the candidate models when the number of potential predictors is large. As a result, similar to linear model selection, penalized approaches have been also developed for linear mixed model selection. Specifically, an appropriate penalized function for the mixed model is carefully designed, while the coefficient estimates can be computed by minimizing the penalized function (or equivalently, maximizing the penalized likelihood). Because of the additional shrinkage requirement for random effects, we are not only penalizing the fixed effects, but also penalizing the random effects. Nevertheless, the covariance matrix of random effects usually contains too many parameters, which excessively increases the complexity of the penalized function. One prominent idea by Ibrahim et al. [2011] is to extend SCAD or adaptive lasso in linear mixed models to penalize fixed and random effects simultaneously. However, as mentioned, the main drawback of this approach is the complexity of locating the global minimum, since the penalized function is somehow complicate. Moreover, the selection of tuning parameters are proceeding separately for fixed and random effects. To improve the computation efficiency, a modified Cholesky decomposition [Chen and Dunson, 2003] is suggested, which simplifies the penalized function by only involving the diagonal elements of the covariance matrix. Following this idea, another competitive approach by only penalizing the fixed effects and diagonal elements of random effects is proposed [Bondell and Ghosh, 2010]. In addition, the selection of tuning parameter is proceeding simultaneously for random and fixed effects in this method, which makes the computation more attractive.

Regarding computation, the problem of maximizing the integral of complete penalized likelihood with respect to random effects, or equivalently, maximizing the marginal likelihood, is difficult to solve directly due to the complex form of the unknown covariance matrix for the random effects. In general, this quantity is intractable, which makes no closed form for the solution 
of the parameter estimates. Hence, EM algorithm is suggested to estimate the parameters regarding penalized likelihood function. The EM algorithm was named and explained by Dempster et al. [1977]. It is an iterative method to find the maximum likelihood estimates of parameters in statistical models, where the model depends on unobserved latent variables. In linear mixed models, the random effects can be treated as unobserved latent variables, and the process keeps alternating between expectation step and maximization step. Specifically, the expectation step evaluates the expectation of penalized log-likelihood with respect to random effects by using the current estimates of all the parameters. Afterwards, the maximization step maximizes the quantity of expected penalized log-likelihood from expectation step, and coordinate descent algorithm is frequently employed when the objective function is high dimensional. The procedure is repeated iteratively between expectation step and maximization step until all parameter estimates converge to some fixed constants.

Following the strategies discussed above, it indicates that linear mixed model selection can still be achieved through the models possess complex forms. Furthermore, some empirical results suggest that a screening step for fixed effects is very likely to improve the selection accuracy, especially for small samples. Therefore, it is recommended to employ a screening step for the candidate models to eliminate possible noise factors.

In this dissertation, we mainly develop a penalized approach to facilitate linear mixed model selection, which effectively chooses the non-zero fixed effects and detect the covariance structure of random effects simultaneously. Moreover, not only theoretical basis is founded to prove that the fitting process possesses model selection consistency, but also a suite of simulation studies are conducted to show that the approach performs quite well empirically. Compared to some existing methods, we demonstrate that the proposed method effectively improves the selection accuracy of fixed effects and keeps a relatively high rate of reflecting the sparsity of random effects. 


\subsection{Outline}

Chapter 2 introduces the general settings of the linear regression models, followed by some popular criterion in model selection. We first illustrate the classical subset methods based on the criterion and then present the penalized approaches. Some typical penalties are addressed and a comparison is made to see the advantages and drawbacks. Specifically, more details are provided for the adaptive lasso, where its extended form is implemented in our algorithm. Meanwhile, crossvalidation is recommended to obtain the optimal choice of tuning parameter. An example of our earlier study is presented to confirm the good performance of adaptive lasso via cross-validation in linear regression models.

In chapter 3, we extend the discussion to linear mixed models. The popular criteria and wellknown penalized approaches addressed in chapter 2 are modified properly to be applied to mixed settings. Considering the complexity caused by additional random effects, a modified Cholesky decomposition is suggested and parameters are reparameterized to simplify the computation. Additionally, the marginal likelihood and complete likelihood of a mixed model are discussed for further use.

Chapter 4 illustrates the details of adaptive lasso via cross-validation in linear mixed models. We focus on the reparameterized model from chapter 3 and show it how to penalize the fixed and random effects simultaneously step by step. Consequently, a reasonable penalized likelihood is obtained, and its maximization can result in a simplified model to achieve the model selection. More specifically, some techniques for attaining the maximum, such as EM algorithm, coordinate descent algorithm are implemented, and a comprehensive view of the computation procedure is

presented. In addition, some theoretical results are founded to ensure the selection consistency and coefficient estimates consistency simultaneously.

In chapter 5, several simulations are conducted. To justify the generality of the method, we investigate the potential changes in model selection accuracy by using different underlying models. In simulations, either normal or uniform distribution is considered to generate the design matrices, 
and the number of variables is adjusted from small to large. Additionally, to detect the possible influence of sample size and subgroup size, we increase the size from moderate small to relatively large. Moreover, we compare the selection results to some existing procedures to demonstrate that the proposed approach performs quite well in linear mixed model selection.

In chapter 6, a real data example is established to show that the practical result is compatible with our earlier analysis.

Finally, an overall conclusion is drawn in chapter 7, and some possible further improvement is discussed as well.

References and selected $\mathrm{R}$ codes are attached in the appendix. 


\section{CHAPTER 2 LINEAR MODEL SELECTION}

\subsection{Linear Regression Models}

Linear regression is a widely used approach for modeling the relationship between a response variable and one or more explanatory variables. Specifically, the response variable in linear regression is a scalar, whereas the response variable in multivariate linear regression is a multivariate variable. In this dissertation, we do not discuss multivariate linear regression.

Considering that there are $n$ observed values for the response variable, namely $y_{1}, y_{2}, \ldots, y_{n}$. Meanwhile, giving that $p$ explanatory variables would affect the response variable, which are denoted as $x_{i 1}, x_{i 2}, \ldots, x_{i p}$, for $i=1,2, \ldots, n$. Following these notations, the model can be formed as

$$
y_{i}=\beta_{1} x_{i 1}+\beta_{2} x_{i 2}+\ldots+\beta_{p} x_{i p}+\epsilon_{i}, \quad i=1,2, \ldots, n
$$

where $\epsilon_{i}$ 's are the error terms. For the simplicity purpose, we often assume that the error terms are independent, and identically distributed with a normal distribution, and this model is also called a Gaussian model.

When matrix notation is preferred, the above formula can be expressed as

$$
\boldsymbol{y}=\mathbf{X} \boldsymbol{\beta}+\boldsymbol{\epsilon},
$$

where each symbol is given by

$$
\boldsymbol{y}=\left(\begin{array}{c}
y_{1} \\
y_{2} \\
\vdots \\
y_{n}
\end{array}\right), \mathbf{X}=\left(\begin{array}{ccc}
x_{11} & \ldots & x_{1 p} \\
x_{21} & \ldots & x_{2 p} \\
\vdots & \ddots & \vdots \\
x_{n 1} & \ldots & x_{n p}
\end{array}\right), \boldsymbol{\beta}=\left(\begin{array}{c}
\beta_{1} \\
\beta_{2} \\
\vdots \\
\beta_{p}
\end{array}\right)
$$


and

$$
\boldsymbol{\epsilon}=\left(\begin{array}{c}
\epsilon_{1} \\
\epsilon_{2} \\
\vdots \\
\epsilon_{n}
\end{array}\right)
$$

For convenience, the matrix notation is frequently used in the next several sections.

\subsection{Subset Methods with Commonly Used Criteria}

\subsubsection{AIC}

The Akaike information criterion [Akaike, 1973] plays a vital role in model selection, which is derived by minimizing the expected value of KullbackLeibler discrepancy when sample size approaches infinity. It has the form:

$$
\mathrm{AIC}=-2 \log f(y \mid \hat{\phi})+2 k
$$

where $\log f(y \mid \hat{\phi})$ is the maximum log-likelihood and $k$ is the number of parameters to be estimated. In practice, smaller AIC indicates a smaller discrepancy, thus the selected model is more likely to be close to the true model. Later study suggests that certain modifications are required when the sample size is small, and $\mathrm{AIC}_{\mathrm{c}}$ [Hurvich and Tsai, 1989] is proposed to adjust the bias for a small sample under normal settings. Since then, $\mathrm{AIC}_{\mathrm{c}}$ has been extended to numerous additional frameworks, including autoregressive moving average model [Hurvich et al., 1990], vector autoregressive model [Hurvich and Tsai, 1993], multivariate linear regression model [Bedrick and Tsai, 1994] and overdispersed generalized linear model [Hurvich and Tsai, 1995]. Nevertheless, further analysis [Shao, 1997] demonstrates that though AIC is asymptotically optimal to minimize the mean squared error loss, it often prefers a more complex model over a simpler model even when the true underlying model is the simpler one. As a result, it is often used with other information 
criteria together to select the true model.

\subsubsection{BIC}

Bayesian information criterion [Schwarz, 1978] is developed for the purpose of maximizing the Bayesian posterior, which takes the form of:

$$
\mathrm{BIC}=-2 \log f(y \mid \hat{\boldsymbol{\phi}})+\log (n) k .
$$

Compared to AIC, the coefficient of the second term in (2.4) is changed to $\log (n)$, which makes BIC favor a smaller model because of the increasing weight. Similar to AIC, a model with smaller BIC is always preferred. In addition, it serves as a consistent criterion in model selection, which solves the over selection problem of AIC. Consequently, BIC is frequently applied when the objective is to detect the true model structure.

\subsubsection{Subset Methods}

Regarding subset methods, it is to choose the set with "best" criterion out of all the possible sets. After determining the subset, least squares regression is frequently used to obtain the coefficient estimates of each variable. To choose the "best" subset, a variety of strategies are considered and forward stepwise and backward stepwise selections are the two most commonly used approaches among them.

Forward stepwise selection starts from the null model and sequentially adds one variable that improves the fit most in each step. This process is terminated while no additional variables result in significant improvement of fit. On the contrary, backward stepwise starts with the full model and deletes one variable with the least significant influence on the model in each step. This elimination process is stopped until no further variables would make statistically significant loss of fit. To justify whether the influence of each step is significant, an appropriate test could be conducted, such as F-test, to determine if the addition or elimination of the variable significantly changes the fit. Alternatively, model selection criteria can be considered as the decision rule for each step. 
Either by forward or backward direction, the stepwise selection is frequently implemented to choose the "best" subset. However, some drawbacks of this method are distinct. Firstly, the tests in each step may be biased [Rencher and Pun, 1980], hence the determination of the significance of adding or dropping a variable is questionable. Secondly, when the test is conducted, only the number of variables of the final fitted model is used for estimating the degrees of freedom, instead of the entire model [Hurvich and Tsai, 1990]. Additionally, the fitting process is unstable due to its discreetness [Breiman, 1996], therefore a small change of the data set may greatly affect the final model. Meanwhile, as illustrated in the introduction, subset methods lack computation efficiency for a high dimensional model.

To correct those deficiencies, penalized approaches are developed to select models from another aspect. In the next several sections, we briefly present some classic penalized approaches and their main properties.

\subsection{Selection Approaches via Penalized Method}

\subsubsection{Penalized Function}

Penalized approaches are popular for selecting models in linear regression models due to its computational feasibility and statistical accuracy [Schelldorfer et al., 2011], especially when the number of predictors is of medium or large size.

Recall the ordinary least squares estimator of linear regression models, the coefficient vector is solved by

$$
\hat{\boldsymbol{\beta}}^{\text {ols }}=\operatorname{argmin}_{\boldsymbol{\beta}} \frac{1}{n}(\boldsymbol{y}-\mathbf{X} \boldsymbol{\beta})^{\prime}(\boldsymbol{y}-\mathbf{X} \boldsymbol{\beta}) .
$$

Consequently, $\hat{\boldsymbol{\beta}}^{\text {ols }}=\left(\mathbf{X}^{\prime} \mathbf{X}\right)^{-1} \mathbf{X}^{\prime} \boldsymbol{y}$ when the design matrix is full column rank. When the design matrix is not full column rank, the inverse of $\mathbf{X}^{\prime} \mathbf{X}$ can be replaced with its generalized inverse.

Compared to the optimization problem for ordinary least squares estimator, penalized ap- 
proaches involve an additional penalty term, which make the optimization problem as

$$
\hat{\boldsymbol{\beta}}^{p l s}=\operatorname{argmin}_{\boldsymbol{\beta}} \frac{1}{n}(\boldsymbol{y}-\mathbf{X} \boldsymbol{\beta})^{\prime}(\boldsymbol{y}-\mathbf{X} \boldsymbol{\beta})+\operatorname{penalty}(\boldsymbol{\beta}) .
$$

where $\hat{\boldsymbol{\beta}}^{\text {pls }}$ is the penalized least squares estimator. To identify the true sparse pattern and guarantee the coefficients' estimation accuracy simultaneously, a penalized method is encouraged to possess the following properties [Fan and Lv, 2001]:

1. Unbiasedness: the estimator is nearly unbiased when the true unknown parameter is relatively large to avoid excessive estimation bias.

2. Sparsity: shrink a small coefficient to zero to reduce model complexity.

3. Continuity: the estimator is continuous for the data to avoid instability in model selection.

Moreover, it also points out that the procedure is good if it follows the oracle property:

1. Identifies the true subset: $P\left(\left\{j: \hat{\beta}_{j}(\delta) \neq 0\right\}=A\right) \rightarrow 1$.

2. Possesses the optimal estimation rate: $\sqrt{n}\left(\hat{\beta}(\delta)-\beta_{0}\right) \rightarrow_{d} N\left(0, \Sigma^{*}\right)$.

For the notations in the above properties, $A$ is the true non-zero subset, $\delta$ is the fitting procedure, $\hat{\beta}_{j}$ is the $j$ th element of the estimator, $\beta_{0}$ is the true coefficient vector and $\Sigma^{*}$ is the covariance matrix knowing the true subset model. Since lasso lays a foundation for all the later penalized methods, we will first discuss lasso method.

\subsubsection{Lasso}

Lasso estimator is defined by solving the optimization problem:

$$
\hat{\boldsymbol{\beta}}^{\text {lasso }}=\operatorname{argmin}_{\boldsymbol{\beta}} \frac{1}{n}(\boldsymbol{y}-\mathbf{X} \boldsymbol{\beta})^{\prime}(\boldsymbol{y}-\mathbf{X} \boldsymbol{\beta})+\lambda\|\boldsymbol{\beta}\|_{1},
$$

where the penalty term is the product of a tuning parameter $\lambda$ and the $L_{1}$ norm of $\boldsymbol{\beta}$. In general, there is no closed form for lasso estimator, which makes the shrinkage difficult to understand. 
However, there exists an explicit closed form when the design matrix is orthogonal. Considering that $\frac{1}{n} \mathbf{X}^{\prime} \mathbf{X}=\mathbf{I}$, the $j$ th element of lasso estimator takes the form:

$$
\hat{\beta}_{j}^{\text {lasso }}=\operatorname{sign}\left(\hat{\beta}_{j}\right)\left(\left|\hat{\beta}_{j}\right|-\frac{\lambda}{2}\right)_{+} .
$$

In this formula, $\operatorname{sign}(\bullet)$ represents the sign function and $\hat{\beta}_{j}$ is the $j$ th element of $\hat{\boldsymbol{\beta}}^{\text {ols }}$ in $(2.5)$. Therefore, the value of $\operatorname{sign}\left(\hat{\beta}_{j}\right)$ equals 1 when $\hat{\beta}_{j}$ is positive, and equals -1 when $\hat{\beta}_{j}$ is negative. Meanwhile, $(\bullet)_{+}$represents the positive part of a real number, then $(x)_{+}=x$ when $\mathrm{x}$ is positive and $(x)_{+}=0$ when $\mathrm{x}$ is non-positive. Clearly, the second term indicates that the $j$ th element of lasso estimate is shrunk to zero if the absolute value of its corresponding element of the ordinary least squares estimate is less than $\frac{\lambda}{2}$. As a result, by adjusting $\lambda$, we can wisely eliminate some variables.

Note that lasso actually achieves model selection by playing the bias-variance trade-off, and with a simpler model, it often improves model selection accuracy. Moreover, when certain conditions are met and the tuning parameter is properly chosen, lasso approach could specify the correct sparse pattern with probability 1 [Donoho and Elad, 2002], and coefficient estimates are consistent [Meinshausen and Bhlmann, 2004]. In another words, the bias could be ignored in some sense. In addition, lasso penalty takes a convex form, which makes the optimization attainable from all paths. However, a regular quadratic program cannot solve it directly due to the non-differentiable points, and some modifications are required.

\subsubsection{Least Angle Regression}

To avoid the discussion of non-differentiable points and maintain the computation efficiency, least angle regression algorithm [Efron et al., 2004] is suggested as an alternative way to obtain the lasso estimate. The algorithm is given by 
1. Start with all coefficients equal to zero and find the predictor most correlated with the response, say $\boldsymbol{x}_{j 1}$.

2. Take the largest step possible in the direction of this predictor until some other predictor, say $\boldsymbol{x}_{j 2}$, has the same correlation with the current residual.

3. Proceed in a direction equiangular between $x_{j 1}$ and $x_{j 2}$ until a third variable $x_{j 3}$ has the same correlation with the current residual corresponding to $\boldsymbol{x}_{j 1}$ and $\boldsymbol{x}_{\boldsymbol{j} 2}$

4. Proceed in a direction equiangular between $\boldsymbol{x}_{j 1}, \boldsymbol{x}_{j 2}$ and $\boldsymbol{x}_{j 3}$ until a fourth variable enters, and continue the process until all the predictors enter the model.

Known as the LARS algorithm, least angle regression can be regarded as a variation of the stepwise selection. The regular stepwise selection is too greedy in each step, which may eliminate a useful predictor from the second step that happens to be highly correlated with the first selected predictor $\boldsymbol{x}_{j 1}$. Compared to the stepwise selection, LARS is a much safer algorithm. To obtain the whole solution path of lasso estimator, we just need to make some adjustments to the LARS algorithm. Specifically, a non-zero coefficient is removed from the active set of predictors if it hits zero, and the new equiangular direction is recomputed from the remaining predictors of the active set. In this way, some variables are dropped, and a simplified model is achieved.

\subsubsection{Coordinate Descent Algorithm}

More recently, coordinate descent algorithm is demonstrated to be a competitive method with the LARS algorithm, particularly in high dimensions [Friedman et al., 2007, Wu and Lange, 2008]. In addition, it is not only efficient for the lasso, but also applicable for a variety of additive penalties [Friedman et al. 2009]. For instance, let the objective penalized function of $\boldsymbol{\beta}$ be $f(\boldsymbol{\beta})$, and to obtain the minimizer, we implement coordinate descent algorithm by starting with a initial guess of $\boldsymbol{\beta}$, namely $\boldsymbol{\beta}^{(0)}$. Afterwards, it minimizes $f(\boldsymbol{\beta})$ with respect to each coordinate at a time, through $\beta_{1}, \beta_{2}, \ldots$ until $\beta_{p}$. A whole cycle of the updates can be expressed as follows: 
1. $\beta_{1}^{(1)}=\operatorname{argmin}_{\beta} f\left(\beta, \beta_{2}^{(0)}, \beta_{3}^{(0)}, \ldots, \beta_{p}^{(0)}\right)$,

2. $\beta_{2}^{(1)}=\operatorname{argmin}_{\beta} f\left(\beta_{1}^{(1)}, \beta, \beta_{3}^{(0)}, \ldots, \beta_{p}^{(0)}\right)$,

p. $\beta_{p}^{(1)}=\operatorname{argmin}_{\beta} f\left(\beta_{1}^{(1)}, \beta_{2}^{(1)}, \beta_{3}^{(1)}, \ldots, \beta\right)$,

and from step 1 to $\mathrm{p}$ until convergence is reached. In each step, the solution of the univariate case is simply given by the soft-thresholding solution, similar to the orthogonal design matrix, but with dimension one. Considering the high computation efficiency of coordinate descent algorithm, we extend it to be further applied for any additive penalty in linear mixed models.

Although lasso is an efficient algorithm to eliminate noise factors, some conditions are easily violated. Therefore, the selection may fail in many cases. On the other hand, recall the three advocated properties: unbiasedness, sparsity, and continuity, lasso approach violates the unbiasedness property for large coefficients. In order to develop an oracle procedure satisfying the advocated properties, Fan and LV [2001] propose the smoothly clipped absolute deviation penalty, which is known as SCAD.

\subsubsection{SCAD}

SCAD penalty takes the form:

$$
p_{\lambda}^{S C A D}(\beta)= \begin{cases}\lambda|\beta|, & \text { if }|\beta| \leq \lambda \\ -\frac{|\beta|^{2}-2 a \lambda|\beta|+\lambda^{2}}{2(a-1)}, & \text { if } \lambda<|\beta| \leq a \lambda \\ \frac{(a+1) \lambda^{2}}{2} . & \text { if }|\beta|>\lambda\end{cases}
$$

Specifically, in this formula, $a$ is constant greater than 2 and $\lambda$ is a tuning parameter greater than 0 . More recent research shows that SCAD is not the only penalty satisfying the three good properties, there are also some other penalties, such as Minimax Concave Penalty [Zhang, 2010] etc.

Nevertheless, either for SCAD or MCP, the penalty term is not globally convex, which makes the global minimum of the penalized function difficult to be determined. For the sake of computa- 
tional convenience, convex penalties are often preferred, then it is natural that statisticians desire to develop a procedure with a convex penalty. Following this idea, adaptive lasso [Zou, 2006] is proposed as a competitive method.

\subsubsection{Adaptive Lasso}

Considering the inconsistency of lasso in many cases, Zou [2006] proposes adaptive lasso, to extend the availability of L-1 norm penalized method in a more general setting. Adaptive Lasso estimator is given by

$$
\hat{\boldsymbol{\beta}}^{\text {alasso }}=\operatorname{argmin}_{\boldsymbol{\beta}} \frac{1}{n}(\boldsymbol{y}-\mathbf{X} \boldsymbol{\beta})^{\prime}(\boldsymbol{y}-\mathbf{X} \boldsymbol{\beta})+\lambda \sum_{i=1}^{p} \hat{w}_{i}\left|\beta_{i}\right|,
$$

where the weights $\hat{w}_{i}=\frac{1}{\left|\hat{\beta_{j}}\right|^{\gamma}}$ for any $\gamma>0$. Unlike lasso penalty, which equally penalizes on all the effects, adaptive lasso assigns different weights for different effects. It is proved that adaptive lasso satisfies the oracle property when the tuning parameters are appropriately chosen with mild regularity conditions compared to lasso. Additionally, the penalty term of adaptive lasso is convex, which makes the computation more attractive. Since adaptive lasso shares many advantages of lasso, most computation techniques for lasso can be extended to adaptive lasso with moderate corrections. In particular, coordinate descent algorithm can be applied with adaptive lasso penalty.

According to the above analysis, adaptive lasso is adopted in our previous research for the model selection in linear regression models (in the introduction). Combining it with cross-validation and a screen step, it performs even better to identify the true model under the regular normal setting (see Table 1.1 and 1.2). As a result, we focus more on extending adaptive lasso in linear mixed models, which will be discussed in chapter 3 .

\subsubsection{Tuning Parameter Selection}

In the previous section, we have seen that the penalty term always involves a tuning parameter $\lambda$. However, not all the tuning parameters can keep the estimation consistency, while in most cases, it must keep a certain growth rate corresponding to the sample size $n$. In the other words, the tuning 
parameter can neither increase too rapidly nor too slowly. In practice, we normally allow the tuning parameter shifting in a certain grid.

Among all the possible tuning parameters in the preassigned grid, we want to select the one which minimizes the loss, and cross-validation is one of the most common approaches. Nevertheless, Wasserman and Roeder [2009] conjectures that implementing cross-validation to minimize mean squared error loss may result in an overspecified model with probability one in model selection. By replacing the mean squared error loss with BIC criteria, simulation results in or study demonstrate that selection accuracy is improved. Consequently, we will consider implementing cross-validation with BIC criteria to select the tuning parameter in linear mixed models. 


\section{CHAPTER 3 LINEAR MIXED MODEL SELECTION}

\subsection{Linear Mixed Models}

A linear mixed model is an extension to a linear regression model, where explanatory variables contain random effects other than fixed effects. Considering the case of $m$ subjects and each subject is observed $n_{i}$ times with $p$ fixed predictors and $q$ random predictors, the linear form of the $i$ th subject is given by

$$
\boldsymbol{y}_{\boldsymbol{i}}=\mathrm{X}_{\mathrm{i}} \boldsymbol{\beta}+\mathrm{Z}_{\mathrm{i}} \boldsymbol{b}_{\boldsymbol{i}}+\boldsymbol{\epsilon}_{\boldsymbol{i}},
$$

where

$$
\boldsymbol{y}_{\boldsymbol{i}}=\left(\begin{array}{c}
y_{i 1} \\
y_{i 2} \\
\vdots \\
y_{i n_{i}}
\end{array}\right), \mathbf{X}_{\mathbf{i}}=\left(\begin{array}{cccc}
x_{i 11} & x_{i 12} & \ldots & x_{i 1 p} \\
x_{i 21} & x_{i 22} & \ldots & x_{i 2 p} \\
\vdots & & & \\
x_{i n_{i} 1} & x_{i n_{i} 2} & \ldots & x_{i n_{i} p}
\end{array}\right), \boldsymbol{\beta}=\left(\begin{array}{c}
\beta_{1} \\
\beta_{2} \\
\vdots \\
\beta_{p}
\end{array}\right)
$$

and

$$
\mathbf{Z}_{\mathbf{i}}=\left(\begin{array}{cccc}
z_{i 11} & z_{i 12} & \ldots & z_{i 1 q} \\
z_{i 21} & z_{i 22} & \ldots & z_{i 2 q} \\
\vdots & & & \\
z_{i n_{i} 1} & z_{i n_{i} 2} & \ldots & z_{i n_{i} q}
\end{array}\right), \boldsymbol{b}_{\boldsymbol{i}}=\left(\begin{array}{c}
b_{i 1} \\
b_{i 2} \\
\vdots \\
b_{i q}
\end{array}\right), \boldsymbol{\epsilon}_{\boldsymbol{i}}=\left(\begin{array}{c}
\epsilon_{i 1} \\
\epsilon_{i 2} \\
\vdots \\
\epsilon_{i n_{i}}
\end{array}\right)
$$

In parallel to linear regression models, we adopt the assumptions that $\boldsymbol{b}_{\boldsymbol{i}}{ }^{\prime} s$ are independent and identically distributed with a multivariate normal distribution. In addition, the error terms $\epsilon_{i}{ }^{\prime} s$ are independent to the random effects $\boldsymbol{b}_{\boldsymbol{i}}{ }^{\prime} s$, and any $\boldsymbol{\epsilon}_{\boldsymbol{i}}$ and $\boldsymbol{\epsilon}_{j}$ are also independent and identically 
distributed. Assuming that some covariates in $\boldsymbol{b}_{\boldsymbol{i}}$ could be correlated and any two covariates in $\boldsymbol{\epsilon}_{\boldsymbol{i}}$ are uncorrelated, the distribution of $\boldsymbol{b}_{i}$ and $\epsilon_{i}$ are given by

$$
\boldsymbol{b}_{\boldsymbol{i}} \sim \mathrm{N}\left(0, \sigma^{2} \boldsymbol{\Psi}\right), \boldsymbol{\epsilon}_{\boldsymbol{i}} \sim \mathrm{N}\left(0, \sigma^{2} \mathbf{I}_{\mathbf{n}_{\mathbf{i}}}\right)
$$

where $\boldsymbol{\Psi}$ is a $n_{i}$ by $n_{i}$ semi-positive definite matrix and $\mathbf{I}_{\mathbf{n}_{\mathbf{i}}}$ is a $n_{i}$ by $n_{i}$ identity matrix.

Given the full data, denote $N=\sum_{i=1}^{m} n_{i}$ as the total number of observed values. Let $\boldsymbol{y}=$ $\left(\boldsymbol{y}_{1}{ }^{\prime}, \boldsymbol{y}_{2}{ }^{\prime}, \ldots \boldsymbol{y}_{m}{ }^{\prime}\right)^{\prime}$ be the response values and $\mathbf{X}=\left(\mathbf{X}_{\mathbf{1}}{ }^{\prime}, \mathbf{X}_{\mathbf{2}}{ }^{\prime}, \ldots \mathbf{X}_{\mathbf{m}}{ }^{\prime}\right)^{\prime}$ be the stacked matrix of fixed effects. Regarding the random effects and error terms, let $\mathbf{Z}=\operatorname{diag}\left(\mathbf{Z}_{\mathbf{1}}, \mathbf{Z}_{\mathbf{2}}, \ldots, \mathbf{Z}_{\mathbf{m}}\right)$, $\boldsymbol{b}=\left(\boldsymbol{b}_{\mathbf{1}}{ }^{\prime}, \boldsymbol{b}_{\mathbf{2}}{ }^{\prime}, \ldots \boldsymbol{b}_{\boldsymbol{m}}{ }^{\prime}\right)^{\prime}$ and $\boldsymbol{\epsilon}=\left(\boldsymbol{\epsilon}_{\mathbf{1}}{ }^{\prime}, \boldsymbol{\epsilon}_{\mathbf{2}}{ }^{\prime}, \ldots \boldsymbol{\epsilon}_{\boldsymbol{m}}{ }^{\prime}\right)^{\prime}$. Hence $\boldsymbol{y}$ is a $N \times 1$ vector, $\mathbf{X}$ is a $N \times p$ matrix and $\mathbf{Z}$ is a $N \times m q$ matrix. For the effects and error terms, $\boldsymbol{\beta}$ is a $p \times 1$ fixed vector, $\boldsymbol{b}$ is a $m q \times 1$ random vector and $\epsilon$ is a $N \times 1$ random vector, where all the values are unknown practically. Following these notations, the whole data has the matrix form:

$$
\boldsymbol{y}=\mathbf{X} \boldsymbol{\beta}+\mathbf{Z} \boldsymbol{b}+\boldsymbol{\epsilon}
$$

Note that the parameters of interest are all the elements in $\boldsymbol{\beta}, \Psi$, and $\sigma^{2}$. Since $\boldsymbol{\Psi}$ is symmetric,

it suffices to consider all the upper triangular elements, which includes $\frac{(q+1) q}{2}$ elements. For convenience, let $\boldsymbol{\theta}$ be the parameters including in $\Psi$ and $\phi$ be the whole parameter vector, then $\phi$ is expressed as $\phi=\left(\boldsymbol{\beta}, \boldsymbol{\theta}, \sigma^{2}\right)$.

\subsection{Commonly Used Criteria in Linear Mixed Models}

An extension of the AIC criteria to linear mixed models is suggested by Sugiura [1978], known as the marginal AIC. In addition to fixed effects, marginal AIC considers random effects and involves an adjustment coefficient. It takes the form:

$$
\operatorname{mAIC}=-2 \log f(y \mid \hat{\phi})+2 a_{N}(p+q)
$$


where $a_{N}$ either takes the value of 1 or $\frac{N}{N-p-q-1}$. Nevertheless, recent research indicates that mAIC is positively biased [Greven and Kneib, 2010] in some cases, and proper modifications are required.

An alternative variation of AIC, conditional Akaike information criterion [Vaida and Blanchard, 2005] is suggested while treating the unknown random effects as parameters. Known as cAIC, this criterion is built under the framework of best linear unbiased predictor [Henderson, [1950]. When the variance parameters are known, random effects are predicted with the best linear unbiased predictor, and cAIC takes the form:

$$
\operatorname{cAIC}=-2 \log f(\hat{\boldsymbol{\phi}} \mid \hat{\boldsymbol{b}})+a_{N}(\hat{\boldsymbol{\phi}}),
$$

where $\hat{\phi}$ is the parameter estimate, $\hat{b}$ is the predicted random effect and $a_{N}(\hat{\phi})$ is the penalty term. Vaida and Blanchard suggest using the penalty term $\frac{2 N}{N-p-2}\left\{p(\hat{\tau})+1-\frac{p(\hat{\tau})-p}{N-p}\right\}$ when the covariance matrix of error term is an identity matrix, where $p(\hat{\tau})$ in the penalty term measures the effect of estimating $\boldsymbol{\beta}$ and $\boldsymbol{b}$.

Regarding the extension of BIC criterion, marginal BIC is developed to extend BIC to linear mixed models, which has the form:

$$
\operatorname{mBIC}=-2 \log f(y \mid \hat{\phi})+\log (n)(p+q) .
$$

Either cAIC or mBIC is frequently implemented in the linear mixed models, and mBIC is showed to often outperform cAIC when the goal is to select the true model. Therefore, we may consider using $\mathrm{mBIC}$ to investigate the true structure of underlying models. 


\subsection{Log-Likelihood Function in Linear Mixed Models}

First, for the $i$ th subject, let $\mathbf{V}_{i}=\sigma^{2}\left(\mathbf{Z}_{i} \Psi \mathbf{Z}_{i}^{\prime}+\mathbf{I}_{n_{i}}\right)$. When the constant term is dropped, the log-likelihood takes the form:

$$
L_{i}(\boldsymbol{\phi})=-\frac{1}{2} \log \left|\mathbf{V}_{i}\right|-\frac{1}{2}\left(\boldsymbol{y}_{i}-\mathbf{X}_{i} \boldsymbol{\beta}\right)^{\prime} \mathbf{V}_{i}^{-1}\left(\boldsymbol{y}_{i}-\mathbf{X}_{i} \boldsymbol{\beta}\right)
$$

Considering the assumption that all the subjects are independent, the log-likelihood of the whole data is

$$
L(\boldsymbol{\phi})=-\frac{1}{2} \log |\tilde{\mathbf{V}}|-\frac{1}{2}(\boldsymbol{y}-\mathbf{X} \boldsymbol{\beta})^{\prime} \tilde{\mathbf{V}}^{-1}(\boldsymbol{y}-\mathbf{X} \boldsymbol{\beta}) .
$$

In equation (3.7), $\tilde{\mathbf{V}}=\operatorname{diag}\left(\mathbf{V}_{1}, \mathbf{V}_{2}, \ldots, \mathbf{V}_{m}\right)$, which is a block diagonal matrix. In particular, note that random effects $\boldsymbol{b}_{\boldsymbol{i}}{ }^{\prime} s$ are not appearing in the log-likelihood, and this is called marginal log-likelihood.

On the contrary, when the random effects $\boldsymbol{b}_{\boldsymbol{i}}{ }^{\prime} s$ are treated as observed values, the log-likelihood of whole data has the form:

$$
L_{c}(\boldsymbol{\phi})=-\frac{N+m q}{2} \log \sigma^{2}-\frac{1}{2} \sigma^{2}\left[(\mathbf{Y}-\mathbf{X} \boldsymbol{\beta}-\mathbf{Z} \boldsymbol{b})^{\prime}(\mathbf{Y}-\mathbf{X} \boldsymbol{\beta}-\mathbf{Z} \boldsymbol{b})+\boldsymbol{b}^{\prime} \boldsymbol{b}\right]
$$

where $L_{c}(\phi)$ is called complete log-likelihood.

\subsection{Cholesky Decomposition}

Cholesky decomposition states that every positive definite matrix A can be decomposed as the product of a lower triangular matrix $\mathbf{L}$ and its conjugate transpose $\mathbf{L}^{*}$ [Cholesky, 1910], namely:

$$
\mathbf{A}=\mathbf{L L}^{*}
$$


When $\mathbf{A}$ is a real matrix, $\mathbf{L}$ has all real entries and the factorization can be written as:

$$
\mathbf{A}=\mathbf{L L}^{\prime}
$$

where $\mathbf{L}^{\prime}$ is the regular transpose. Recall the covariance matrix $\Psi$ in linear mixed models is always positive definite, then $\Psi$ could be decomposed as the product of a lower triangular matrix and its transpose. Specifically, let $\mathbf{R}$ denote the real lower triangular matrix, covariance matrix is decomposed as

$$
\Psi=\mathbf{R R}^{\prime}
$$

To separate the parameters of interest of the covariance matrix to the nuisance parameters, Chen and Dunson [2003] suggest a modified Cholesky decomposition to decompose the covariance matrix as

$$
\Psi=\mathrm{DTT}^{\prime} \mathrm{D}
$$

where $\mathbf{D}=\operatorname{diag}\left(d_{1}, d_{2}, \ldots d_{q}\right)$ is a diagonal matrix and $\mathrm{T}$ is a lower triangular matrix with $1^{\prime} s$ on the diagonal. The advantage of writing the covariance matrix in this way is to relate the $i$ th random effect to the $i$ th diagonal element of the matrix, which tremendously simplifies the selection complexity. Specifically, to include the $i$ th predictor is equivalent to obtain a non-zero estimate of the $i$ th diagonal element, regardless of the non-diagonal elements; likewise, to drop the $i$ th predictor is equivalent to estimate the $i$ th diagonal element as zero. In the next section, we adopt this modified Cholesky decomposition to transform the parameters in the covariance matrix and reinterpret the model.

\subsection{Parameter Transformation}

By adopting the modified Cholesky decomposition, covariance matrix is factorized as $\Psi=$ $\mathrm{DTT}^{\prime} \mathrm{D}$, thus the linear mixed model (3.1) can be written as

$$
Y_{i}=\mathrm{X}_{\mathrm{i}} \boldsymbol{\beta}+\mathrm{Z}_{\mathrm{i}} \mathrm{DT} b_{i}^{*}+\epsilon_{i}
$$


where $\boldsymbol{b}_{\boldsymbol{i}}^{*}$ follows a standard normal distribution $N_{q}\left(0, \sigma^{2} \mathbf{I}_{\mathbf{n}_{\mathbf{i}}}\right)$. Let $\boldsymbol{d}=\left(d_{1}, d_{2}, \ldots d_{q}\right)$ be the collection of diagonal elements of $\mathbf{D}$ and $\gamma=\left(\gamma_{i j}\right)$ be the collection of lower triangular elements of $\mathbf{T}$, where $\gamma_{i j}$ is the $(i, j)$ th element of $\mathbf{T}(i=2, \ldots q ; j=1, \ldots i-1)$. Following these notations, the parameters of interest $\phi$ can be expressed as $\phi=\left(\boldsymbol{\beta}, \boldsymbol{d}, \boldsymbol{\gamma}, \sigma^{2}\right)$, and the total number of unknown parameters is $p+q+\frac{(q-1) q}{2}+1$.

Regarding the full data, linear mixed model (3.2) is given by

$$
\boldsymbol{Y}=\mathbf{X} \boldsymbol{\beta}+\mathbf{Z} \tilde{\mathbf{D}} \tilde{\mathbf{T}} b^{*}+\boldsymbol{\epsilon}
$$

where $\tilde{\mathbf{D}}=\operatorname{diag}(\mathbf{D}, \mathbf{D}, \ldots \mathbf{D}), \tilde{\mathbf{T}}=\operatorname{diag}(\mathbf{T}, \mathbf{T}, \ldots \mathbf{T})$, and both of them are $m q \times m q$ block diagonal matrices. Meanwhile, as mentioned, the $i$ th random effect only depends on the $i$ th element of $\boldsymbol{d}$, which is $d_{i}$. Therefore, the selection of significant random effects turns to identify all the nonzero elements in $\boldsymbol{d}$.

\subsection{Selection Approaches via Penalized Method in Linear Mixed Models}

Some methods have been proposed to achieve the model selection in linear mixed models, such as REML and Pathwise Coordinate Optimization [Foster et al., 2007], Automatic Model Selection for Partially Linear Models [Ni et al., 2010] etc.. Nevertheless, those approaches are only capable to select fixed effects when random effects are not subject to the selection.

To jointly select the fixed and random effects, Ibrahim et al. [2011] suggest maximizing the penalized log-likelihood

$$
Q_{1}(\phi)=L(\phi)-m \sum_{i=1}^{p} \phi_{\lambda_{i}}\left(\left|\beta_{i}\right|\right)-m \sum_{j=1}^{q} \phi_{\lambda_{p+j}}\left(|| \boldsymbol{\gamma}_{j} \|\right)
$$

In equation (3.12), $L(\phi)$ is the marginal $\log$-likelihood defined in (3.7), $\beta_{i}{ }^{\prime} s$ are the coefficients of fixed effects and $\gamma_{j}{ }^{\prime} s$ are the column vectors including all the nonzero elements in the $j$ th row of 
$\boldsymbol{R}$ ( Recall $\boldsymbol{R}$ is the Cholesky decomposed matrix of the covariance matrix $\boldsymbol{\Psi}$, defined in equation (3.9) ). In terms of the tuning parameters, $\lambda_{i}$ and $\lambda_{p+j}$ are assigned in different manners, where $\lambda_{i}$ is a constant regardless of the index $i$, while $\lambda_{p+j}$ varies with respect to the index $j$. For the function $\phi$, it either takes SCAD penalty or adaptive lasso penalty.

To simplify the selection of tuning parameters, Bondell and Ghosh [2010] propose maximizing the penalized function

$$
Q_{2}(\boldsymbol{\phi})=-E\left[(\mathbf{Y}-\mathbf{X} \boldsymbol{\beta}-\mathbf{Z} \boldsymbol{b})^{\prime}(\mathbf{Y}-\mathbf{X} \boldsymbol{\beta}-\mathbf{Z} \boldsymbol{b})+\boldsymbol{b}^{\prime} \boldsymbol{b}\right]-\lambda_{m}\left(\sum_{j=1}^{p} \frac{\left|\beta_{j}\right|}{\left|\hat{\beta}_{j}\right|}+\sum_{j=1}^{q} \frac{\left|d_{j}\right|}{\left|\hat{d}_{j}\right|}\right),
$$

where $\hat{\beta}_{j}^{\prime} s$ are the generalized least squares estimates of fixed effects and $\hat{d}_{j}^{\prime} s$ are the restricted maximum likelihood estimates of diagonal matrix $\mathbf{D}$ for the covariance matrix. The function $E(\bullet)$ represents the expectation with respect to $b$. Compared to (3.10), only one tuning parameter is included and we only penalize the fixed effects and the diagonal elements of the covariance. Therefore, the penalized function takes a much simpler form.

In addition to the two approaches mentioned above, an alternative method is given by Peng and Lu [2012]. Two penalized functions are considered to be minimized separately for the random effects and fixed effects. Specifically, regarding the $i$ th subject, the function to be minimized takes the form:

$$
Q_{3}\left(\boldsymbol{b}_{\boldsymbol{i}}\right)=\left(\boldsymbol{Y}_{\boldsymbol{i}}-\mathbf{X}_{\mathbf{i}} \boldsymbol{\beta}-\mathbf{Z}_{\mathbf{i}} \boldsymbol{b}_{\boldsymbol{i}}\right)^{\prime}\left(\boldsymbol{Y}_{\boldsymbol{i}}-\mathbf{X}_{\mathbf{i}} \boldsymbol{\beta}-\mathbf{Z}_{\mathbf{i}} \boldsymbol{b}_{\boldsymbol{i}}\right)+2 N \sum_{i=1}^{q} \phi_{\lambda_{i}}\left(\sqrt{\mid \hat{\psi_{k k} \mid}}\right),
$$

where $\hat{\psi_{k k}}{ }^{\prime} s$ are current the diagonal element estimates of $\boldsymbol{\Psi}$. With the minimizer $\boldsymbol{b}_{\boldsymbol{i}}$ from (3.14), $\hat{\Psi}$ is updated by

$$
\hat{\mathbf{\Psi}}=\frac{\sum_{i=1}^{m} \boldsymbol{b}_{\boldsymbol{i}} \boldsymbol{b}_{\boldsymbol{i}}{ }^{\prime}}{m \hat{\sigma}^{2}}-\frac{\sum_{i=1}^{m} \mathbf{Z}_{\mathbf{i}} \mathbf{Z}_{\mathbf{i}}^{\prime}}{m}
$$

Afterwards, with the estimated block diagonal matrix $\hat{\Psi}_{\mathrm{B}}=\operatorname{diag}(\hat{\Psi}, \ldots, \hat{\Psi})$, the fixed effects are 
updated by minimizing the function

$$
Q_{4}(\boldsymbol{\beta})=(\boldsymbol{Y}-\mathbf{X} \boldsymbol{\beta})^{\prime}\left(\mathbf{I}+\mathbf{Z} \hat{\Psi}_{\mathbf{B}} \mathbf{Z}^{\prime}\right)^{-1}(\boldsymbol{Y}-\mathbf{X} \boldsymbol{\beta})+2 N \sum_{j=1}^{p} \phi_{\lambda_{k}}\left|\beta_{j}\right|
$$

In this approach, the function $\phi(\bullet)$ takes the SCAD penalty, and effect estimates are iteratively updated by (3.14) and (3.15).

For the shrinkage methods mentioned above, the major advantages over direct application of information criteria is reducing the model selection path to choosing a tuning constant [Muller et al., 2013]. Therefore, the selection of tuning constant is particularly important, which will be discussed more thoroughly in our approach. 


\section{CHAPTER 4 ADAPTIVE LASSO VIA CROSS-VALIDATION FOR LINEAR MIXED MODEL SELECTION}

In Chapter 3, we introduce the commonly used criteria and penalized approaches for mixed models. Nevertheless, simulation results indicate that selected model tends to be overfitting for a moderate sample size. Considering the facts that cross-validation effectively decreases the overselection rate for linear regression models, we may naturally want to implement cross-validation in mixed models. In particular, with adaptive lasso penalty, we employ cross-validation to select the "best" tuning parameter which minimizes the mBIC for test data. As a result, some coefficients are shrunk to zeros and simplified model is obtained. We call this approach adaptive lasso via cross-validation, which inherits all the advantages of adaptive lasso while improves the selection accuracy practically by decreasing the over selection rate.

On the other hand, theoretical results are founded to ensure the proposed approach possesses oracle properties under regular conditions. Regarding the computation: Cholesky Decomposition is employed to simplify the penalized function; EM algorithm is implemented to minimize the penalized function, and coordinate descent algorithm is adopted to accelerate the computation speed.

First of all, we start with the construction of penalized objective function.

\subsection{Penalized Function}

Following the notations of linear mixed model in (3.11), penalized adaptive lasso likelihood is given by

$$
Q(\phi)=L(\phi)-\lambda_{m}\left(\sum_{j=1}^{p} \frac{\left|\beta_{j}\right|}{\left|\hat{\beta}_{j}\right|}+\sum_{j=1}^{q} \frac{\left|d_{j}\right|}{\left|\hat{d}_{j}\right|}\right)
$$


where $L(\phi)$ is the marginal likelihood defined in (3.7), $\hat{\beta}_{j}$ is the $j$ th element of generalized least squares estimator of fixed effects and $\hat{d}_{j}$ is the $j$ th element of restricted maximum likelihood estimator of diagonal matrix D. However, as discussed earlier, there is no closed form solution for $\phi$ to maximize $Q(\phi)$. Therefore, to obtain the maximum, we start the analysis with complete likelihood. Treating $b^{*}$ as observed values, complete likelihood of full data is given by

$$
L\left(\operatorname{data} \mid b^{*}, \beta, \theta, \sigma^{2}\right)=(2 \pi)^{-\frac{N}{2}} \sigma^{-N} \exp \left\{-\frac{\left\|Y-X \beta-Z \tilde{D} \tilde{T} b^{*}\right\|^{2}}{2 \sigma^{2}}\right\},
$$

while the prior of $b^{*}$ is

$$
p\left(b^{*} \mid \sigma^{2}\right)=(2 \pi)^{-\frac{m q}{2}} \sigma^{-m q} \exp \left\{-\frac{b^{*^{\prime}} b^{*}}{2 \sigma^{2}}\right\} .
$$

By the Bayesian theorem, posterior density is proportional to the product of likelihood and prior, namely

$$
\operatorname{post}\left(b^{*} \mid \text { data, } \beta, \theta, \sigma^{2}\right) \propto L\left(\text { data } \mid b^{*}, \beta, \theta, \sigma^{2}\right) \times p\left(b^{*} \mid \sigma^{2}\right) .
$$

Dropping the constant regardless of $b^{*}$, we obtain the posterior density as

$$
\operatorname{post}\left(b^{*} \mid \text { data, } \beta, \theta, \sigma^{2}\right) \propto\left(\sigma^{2}\right)^{\frac{N+m q}{2}} \exp \left\{\frac{\left\|Y-X \beta-Z \tilde{D} \tilde{T} b^{*}\right\|^{2}+b^{*^{\prime}} b^{*}}{2 \sigma^{2}}\right\}
$$

Taking logarithm of the posterior density, the log-posterior is given by

$$
l\left(b^{*} \mid \beta, \theta, \sigma^{2}, \text { data }\right)=\text { Constant }-\frac{N+m q}{2} \log \sigma^{2}-\frac{\left\|Y-X \beta-Z \tilde{D} \tilde{T} b^{*}\right\|^{2}+b^{*^{\prime}} b^{*}}{2 \sigma^{2}} .
$$

Given the fact that $b^{*}$ is an unobserved random variable, the original maximization problem now turns to maximize the expectation of $l\left(b^{*} \mid \beta, \theta, \sigma^{2}\right.$, data $)$ with respect to $b^{*}$. Meanwhile, when EM algorithm is implemented, it suffices to consider the conditional expectation of (4.2) under current estimates of $\left(\beta, \theta, \sigma^{2}\right)$ in E-step. Note that only $(\beta, \theta)$ would affect model selection result, we drop the terms regardless of $(\beta, \theta)$, and the maximization of $E\left(l\left(\beta, \theta, \sigma^{2}\right.\right.$, data $\left.\left.\mid b^{*}\right)\right)$ is equivalent to 
minimize the expectation with the form:

$$
E\left\{Q_{0}\left(\beta, \theta, \text { data } \mid b^{*}\right)\right\}=\left.E\right|_{b^{*}}\left\{|| Y-X \beta-\left.Z \tilde{D} \tilde{T} b^{*}\right|^{2},\right.
$$

where "|" means conditional on the known density of $b^{*}$. To shrink some coefficients to zero, adaptive lasso penalty is adopted to penalize $\beta$ and $d$ in $E\left\{Q_{0}\left(\beta, \theta\right.\right.$, data $\left.\left.\mid b^{*}\right)\right\}$, and the objective penalized function $E\left\{Q\left(\beta, \theta\right.\right.$, data $\left.\left.\mid b^{*}\right)\right\}$ takes the form:

$$
E\left\{Q\left(\beta, \theta, \text { data } \mid b^{*}\right)\right\}=\left.E\right|_{b^{*}}\left\{|| Y-X \beta-\left.Z \tilde{D} \tilde{T} b^{*}\right|^{2}+\lambda_{m}\left(\sum_{j=1}^{p} \frac{\left|\beta_{j}\right|}{\left|\hat{\beta}_{j}\right|}+\sum_{j=1}^{q} \frac{\left|d_{j}\right|}{\left|\hat{d}_{j}\right|}\right)\right\}
$$

Specifically, let $\hat{\boldsymbol{d}}=\left(\hat{d}_{1}, \hat{d}_{2}, \ldots \hat{d}_{q}\right)$ be obtained from the restricted maximum likelihood estimates of covariance, then $\hat{\boldsymbol{\beta}}=\left(\hat{\beta}_{1}, \hat{\beta}_{2}, \ldots \hat{\beta}_{p}\right)$ is the generalized least squares estimator with known variance. Recall in equation (4.4), $\beta_{j}$ or $d_{j}$ is the $j$ th element of $\hat{\boldsymbol{\beta}}$ or $\hat{\boldsymbol{d}}$. In practice, $\hat{\beta}$ and $\hat{d}$ can be both estimated by the "Imer" function in R.

\subsection{Tuning Parameter selection via cross-validation}

In the last section, we show that the simplified model is obtained by minimizing the target function $E\left\{Q\left(\beta, \theta\right.\right.$, data $\left.\left.\mid b^{*}\right)\right\}$ in (4.4), which involves the adaptive lasso penalty. In this section, in order to select the "best" tuning parameter, we employ cross-validation with mBIC criterion. To make this procedure more explicit, we illustrate it in two steps. In the first step, for each tuning parameter, we focus on the selection of the candidate model. Thereafter, the second step is concentrated on the evaluation of the candidate model from the first step. A clear comparison is made in diagram 4.1 to show that our approach (the bottom one) reduces the dependency between selecting model and evaluating model, since the two procedures are processing regarding different datasets. 

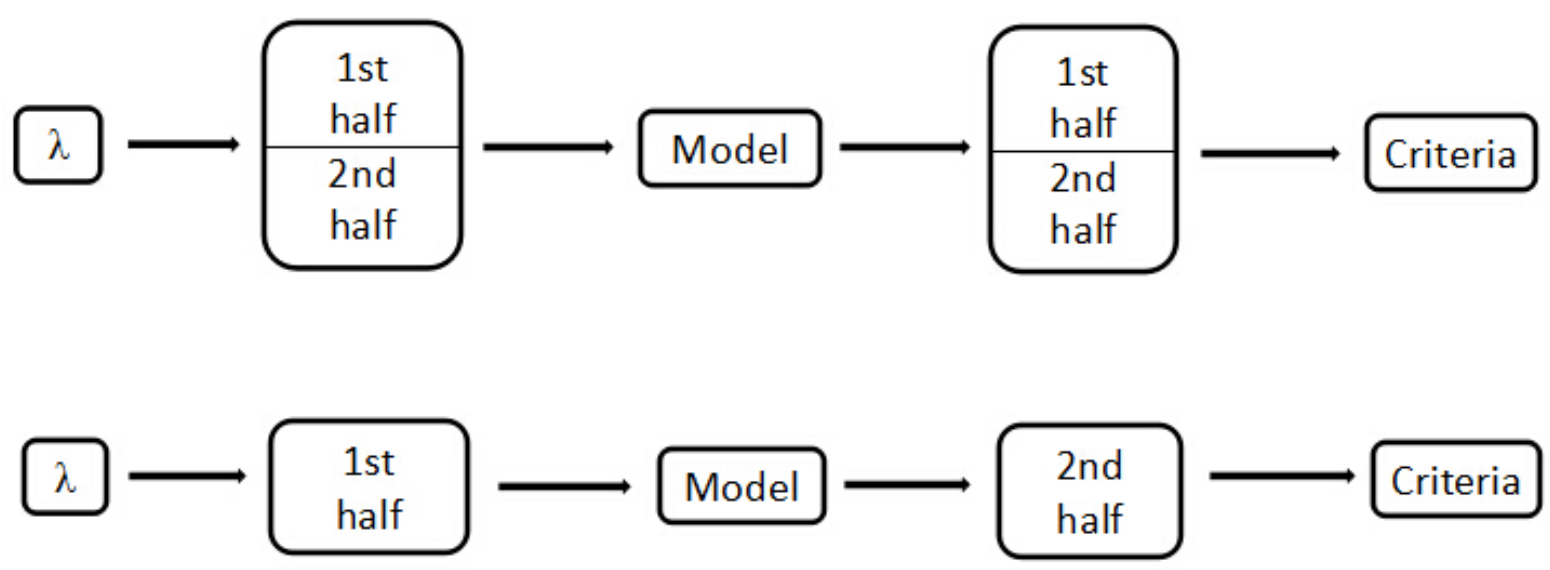

Figure 4.1: Comparing the difference to existing procedures

\subsubsection{Step One}

We start with dividing the whole data into two parts, namely $D_{1}$ and $D_{2}$. Following the notations in (3.2), total sample size is $m$, therefore both $D_{1}$ and $D_{2}$ include $\frac{m}{2}$ subjects. First of all, a tuning parameter $\lambda$ is chosen in the preassigned interval. With the specific $\lambda$, minimizing $E\left\{Q\left(\beta, \theta\right.\right.$, data $\left.\left.\mid b^{*}\right)\right\}$ (the details of the minimization is illustrated in the subsection 4.2.3) for $D_{1}$ with respect to $(\beta, \theta)$, we obtain a candidate model. Since the candidate model depends on the value of $\lambda$, it can be written as $\widehat{S}_{m}(\lambda)$. In general, for all $\lambda$ in the parameter space, the whole set of candidate models can be given as $S=\left\{\widehat{S}_{m}(\lambda): \lambda \in \Lambda\right\}$, where $\Lambda$ is the parameter space of $\lambda$. More specifically, to emphasize that each model is actually determined by its non-zero coefficients, we can express each model $\widehat{S}_{m}(\lambda)$ as

$$
\widehat{S}_{m}(\lambda)=\{\beta, \theta:(\tilde{\beta}, \tilde{\theta})(\lambda) \neq 0\}
$$

where $\tilde{\beta}(\lambda)$ and $\tilde{\theta}(\lambda)$ are the non-zero coefficients. Consequently, by enumerating all $\lambda^{\prime} s$ in the parameter space, all candidate models are obtained, and we proceed to next step to select the "best" model. 


\subsubsection{Step Two}

To evaluate the candidate model's performance, we fit $D_{2}$ with the candidate model $\widehat{S}_{m}(\lambda)$ and compute the corresponding mBIC. As a result, each $\lambda$ determines a candidate model, and each candidate model gives a mBIC value, where the relation can be shown as

$$
\lambda \rightarrow \widehat{S}_{m}(\lambda) \rightarrow \mathrm{mBIC}
$$

Similarly, to emphasize the one-to one relation between tuning parameter $\lambda$ and $\mathrm{mBIC}$, we denote $\operatorname{mBIC}$ as $\operatorname{mBIC}(\lambda)$. Let the "best" tuning parameter be $\lambda^{*}$, then $\lambda^{*}$ is given by

$$
\lambda^{*}=\operatorname{argmin}_{\lambda \in \Lambda} \operatorname{mBIC}(\lambda) .
$$

With the $\lambda^{*}$ from step two, $\widehat{S}_{n}\left(\lambda^{*}\right)$ is also determined, which is considered as the "best" candidate model.

\subsubsection{Minimization}

Now we focus on the minimization of the objective function $E\left\{Q\left(\beta, \theta\right.\right.$, data $\left.\left.\mid b^{*}\right)\right\}$ in step one. Note that $E\left\{Q\left(\beta, \theta\right.\right.$, data $\left.\left.\mid b^{*}\right)\right\}$ is a function with respect to $\beta$ and $\theta$, while the minimizer does not possess a closed form. Consequently, to minimize $E\left\{Q\left(\beta, \theta\right.\right.$, data $\left.\left.\mid b^{*}\right)\right\}$, we employ the EM algorithm. By starting with the initial estimates of $(\beta, \theta)$, namely $\left(\beta^{(0)}, \theta^{(0)}\right)$, we obtain the current estimated matrices $\tilde{D}^{0}$ and $\tilde{T}^{0}$, from which the posterior density of $b^{*}$ can be determined. Furthermore, in the next several paragraphs, we are showing that the posterior actually follows a normal distribution.

Recall log-posterior of $b^{*}$ takes the form of (4.2), and we extract the kernel involving $b^{*}$, which is given by

$$
\operatorname{kernel}\left(b^{*}\right)=\frac{\left\|Y-X \beta-Z \tilde{D} \tilde{T} b^{*}\right\|^{2}+b^{*^{\prime}} b^{*}}{2 \sigma^{2}} .
$$


Treating $\operatorname{kernel}\left(b^{*}\right)$ as a quadratic form of $b^{*}$, it can be also written as

$$
\operatorname{kernel}\left(b^{*}\right)=\left(b^{*}-A^{-1} B^{\prime}\right)^{\prime}\left(A^{-1} \sigma^{2}\right)^{-1}\left(b-A^{-1} B^{\prime}\right)+\text { Constant }
$$

where $A=\left(\tilde{T}^{\prime} \tilde{D} Z^{\prime} Z \tilde{D} \tilde{T}+I\right)$ and $B=(Y-X \beta)^{\prime}(Z \tilde{D} \tilde{T})$. Note that $\operatorname{kernel}\left(b^{*}\right)$ is the kernel of a normal distribution with mean $A^{-1} B^{\prime}$ and variance $A^{-1} \sigma^{2}$, therefore the full expressions of mean and variance are given by

$$
\hat{b}=\left(\tilde{T}^{\prime} \tilde{D} Z^{\prime} Z \tilde{D} \tilde{T}+I\right)^{-1}(Z \tilde{D} \tilde{T})^{\prime}(Y-X \beta)
$$

and

$$
\hat{U}=\sigma^{2}\left(\tilde{T}^{\prime} \tilde{D} Z^{\prime} Z \tilde{D} \tilde{T}+I\right)^{-1}
$$

where $\hat{b}$ is the mean and $\hat{U}$ is the variance. Specifically for the first step, substituting $(\tilde{D}, \tilde{T})$ with the current estimates $\left(\tilde{D}^{0}, \tilde{T}^{0}\right)$, current posterior mean and variance are computed by (4.5) and (4.6). In addition, since $\sigma^{2}$ in (4.6) is unknown, we replace it with its maximum likelihood estimate under the current $\tilde{D}^{0}$ and $\tilde{T}^{0}$. By observing the distribution of $Y$ takes the form

$$
Y \sim N\left(X \beta^{(0)}, \sigma^{2}\left(Z \tilde{D}^{(0)} \tilde{T}^{(0)} \tilde{T}^{(0)^{\prime}} \tilde{D}^{(0)} Z^{\prime}+I\right)\right)
$$

the current estimate of $\sigma^{2}$ can be computed by

$$
{\hat{\sigma^{2}}}^{(0)}=\frac{1}{N}\left(Y-X \beta^{(0)}\right)^{\prime}\left(Z D^{(0)} T^{(0)} T^{(0)^{\prime}} D^{(0)} Z^{\prime}+I\right)\left(Y-X \beta^{(0)}\right),
$$

where $\mathrm{N}$ is the total number of response values.

After specifying the posterior density of $b^{*}$ with the current estimates, the minimization problem can be written as

$$
E_{b^{*} \mid \tilde{D}^{(0)}, \tilde{T}(0)}\left\{|| Y-X \beta-Z \tilde{D} \tilde{T} b^{*}||^{2}+\lambda_{m}\left(\sum_{j=1}^{p} \frac{\left|\beta_{j}\right|}{\left|\hat{\beta}_{j}\right|}+\sum_{j=1}^{q} \frac{\left|d_{j}\right|}{\left|\hat{d}_{j}\right|}\right)\right\} .
$$


Note that (4.8) is a function with respect to $(\boldsymbol{\beta}, \boldsymbol{\theta})$, where $\tilde{D}$ and $\tilde{T}$ are the matrices including parameters $\boldsymbol{\theta}$. More specifically, $\tilde{D}$ contains the parameters $\boldsymbol{d}=\left(d_{1}, d_{2}, \ldots, d_{q}\right), \tilde{T}$ contains the parameters $\boldsymbol{\gamma}=\left(\gamma_{1}, \gamma_{2}, \ldots, \gamma_{q}\right)$, and $\boldsymbol{\theta}$ is the combination of $\boldsymbol{d}$ and $\boldsymbol{\gamma}$, namely $\boldsymbol{\theta}=(\boldsymbol{d}, \boldsymbol{\gamma})$. Therefore, the objective turns to find $(\boldsymbol{\beta}, \boldsymbol{d}, \gamma)$ that minimizes function (4.8). Further analysis indicates that $E_{b^{*} \mid \tilde{D}^{(0)}, \tilde{T}^{(0)}}\left\{\left\|Y-X \beta-Z \tilde{D} \tilde{T} b^{*}\right\|^{2}\right.$ can be written as a quadratic form of $(\boldsymbol{\beta}, \boldsymbol{d})$ when $\gamma$ is known. Alternatively, it can be also expressed as a quadratic term of $\boldsymbol{\gamma}$ when $(\boldsymbol{\beta}, \boldsymbol{d})$ is known. For more details, the explicit forms of both quadratic forms are discussed in the section 4.4. As a result, to obtain the minimum of (4.8), we may iteratively update $(\boldsymbol{\beta}, \boldsymbol{d})$ and $\gamma$ until they converge to some fixed constants.

Next, by observing that the penalty term $\lambda_{m}\left(\sum_{j=1}^{p} \frac{\left|\beta_{j}\right|}{\left|\hat{\beta}_{j}\right|}+\sum_{j=1}^{q} \frac{\left|d_{j}\right|}{\left|\hat{d}_{j}\right|}\right)$ does not include $\gamma$, the minimization of (4.8) with respect to $\gamma$ is no more complicated than a standard minimization problem for the quadratic form, which can be solved by a standard quadratic program. Nevertheless, the penalty term includes parameters $(\boldsymbol{\beta}, \boldsymbol{d})$, which makes standard quadratic program invalid for the minimization of (4.8) regarding $(\boldsymbol{\beta}, \boldsymbol{d})$. In this case, we extend the application of the coordinate descent algorithm, which firstly computes the univariate solution for each coordinate, and then search in different coordinate directions cyclically.

\subsubsection{Univariate Solution for Penalized Quadratic Form}

Let $\boldsymbol{\eta}=(\boldsymbol{\beta}, \boldsymbol{d})$ be a $(p+q) \times 1$ vector, $\mathbf{M}$ be a $(p+q) \times(p+q)$ positive definite matrix, $\boldsymbol{N}$ be a $1 \times(p+q)$ vector, and $\mathrm{L}$ be a penalized quadratic function with the form:

$$
\mathrm{L}=\boldsymbol{\eta}^{\prime} \mathbf{M} \boldsymbol{\eta}-2 \boldsymbol{N} \boldsymbol{\eta}+\sum_{i=1}^{p+q} w_{i}\left|\eta_{i}\right|
$$

where $\eta_{i}$ is the $i$ th element of $\boldsymbol{\eta}$ and $w_{i}$ is the positive weight corresponding to $\eta_{i}$. Taking subderivative of $\mathrm{L}$ with respect to $\eta_{i}$, then sub partial derivative $\nabla_{i} \mathrm{~L}$ is given by

$$
\nabla_{i} \mathrm{~L}=2 \mathbf{M}_{i \bullet} \boldsymbol{\eta}-2 \boldsymbol{N}_{i}+w_{i} \partial\left|\eta_{i}\right|
$$


where $\mathbf{M}_{i \bullet}$ is the $i$ th row of $\mathbf{M}, \boldsymbol{N}_{i}$ is the $i$ th element of $\boldsymbol{N}$ and $\partial\left|\eta_{i}\right|$ is the subderivative of $\left|\eta_{i}\right|$. Splitting $\eta_{i}$ with other covariates in $\boldsymbol{\eta}, \nabla_{i} \mathrm{~L}$ can be written as

$$
\nabla_{i} \mathrm{~L}=2 \sum_{j \neq i} \mathbf{M}_{i j} \eta_{j}+2 \mathbf{M}_{i i} \eta_{i}-2 \boldsymbol{N}_{i}+w_{i} \partial\left|\eta_{i}\right|
$$

To find the minimum of $\mathrm{L}$ corresponding to $\eta_{i}$, it suffices to set $\nabla_{i} \mathrm{~L}=0$. Therefore, we have the equation with respect to $\eta_{i}$ as

$$
-2 \mathbf{M}_{i i} \eta_{i}+\left(2 \boldsymbol{N}_{i}-2 \sum_{j \neq i} \mathbf{M}_{i j} \eta_{j}\right)=w_{i} \partial\left|\eta_{i}\right|
$$

Following the definition of subderivative, $\partial\left|\eta_{i}\right|$ takes the form:

$$
\partial\left|\eta_{i}\right|= \begin{cases}-1, & \text { if } \eta_{i}<0 \\ 1, & \text { if } \eta_{i}>0 \\ {[-1,1] .} & \text { if } \eta_{i}=0\end{cases}
$$

Let $\mathrm{L}$ take the minimum at $\eta_{i}{ }^{*}$, then there are three cases to be considered.

Case 1. When $\eta_{i}{ }^{*}>0$

Equation (4.10) takes the form $-2 \mathbf{M}_{i i} \eta_{i}{ }^{*}+\left(2 \boldsymbol{N}_{i}-2 \sum_{j \neq i} \mathbf{M}_{i j} \eta_{j}-w_{i}\right)=0$.

Solving for $\eta_{j}$, the univariate solution is given as: $\eta_{i}{ }^{*}=\frac{2 \boldsymbol{N}_{i}-2 \sum_{j \neq i} \mathbf{M}_{i j} \eta_{j}-w_{i}}{2 \mathbf{M}_{i i}}$.

Since $\eta_{i}{ }^{*}>0,2 \boldsymbol{N}_{i}-2 \sum_{j \neq i} \mathbf{M}_{i j} \eta_{j}-w_{i}>0$, then $2 \boldsymbol{N}_{i}-2 \sum_{j \neq i} \mathbf{M}_{i j} \eta_{j}>w_{i}$.

Case 2. When $\eta_{i}{ }^{*}<0$.

Equation (4.10) takes the form $-2 \mathbf{M}_{i i} \eta_{i}{ }^{*}+\left(2 \boldsymbol{N}_{i}-2 \sum_{j \neq i} \mathbf{M}_{i j} \eta_{j}+w_{i}\right)=0$.

Solving for $\eta_{j}$, the univariate solution is given as: $\eta_{i}{ }^{*}=\frac{2 \boldsymbol{N}_{i}-2 \sum_{j \neq i} \mathbf{M}_{i j} \eta_{j}+w_{i}}{2 \mathbf{M}_{i i}}$. 
Since $\eta_{i}^{*}<0,2 \boldsymbol{N}_{i}-2 \sum_{j \neq i} \mathbf{M}_{i j} \eta_{j}+w_{i}<0$, then $2 \boldsymbol{N}_{i}-2 \sum_{j \neq i} \mathbf{M}_{i j} \eta_{j}<-w_{i}$.

Case 3. When $\eta_{i}{ }^{*}=0$.

Equation (4.10) takes the form $\left(2 \boldsymbol{N}_{i}-2 \sum_{j \neq i} \mathbf{M}_{i j} \eta_{j}\right) \in\left[-w_{i}, w_{i}\right]$.

To sum up, the soft-thresholding solution of equation (4.10) has the form:

$$
\eta_{i}^{*}= \begin{cases}\frac{2 \boldsymbol{N}_{i}-2 \sum_{j \neq i} \mathbf{M}_{i j} \eta_{j}-w_{i}}{2 \mathbf{M}_{i i}} & \text { if } 2 \boldsymbol{N}_{i}-2 \sum_{j \neq i} \mathbf{M}_{i j} \eta_{j}>w_{i}, \\ \frac{2 \boldsymbol{N}_{i}-2 \sum_{j \neq i} \mathbf{M}_{i j} \eta_{j}+w_{i}}{2 \mathbf{M}_{i i}} & \text { if } 2 \boldsymbol{N}_{i}-2 \sum_{j \neq i} \mathbf{M}_{i j} \eta_{j}<-w_{i}, \\ 0 & \text { if }-w_{i}<2 \boldsymbol{N}_{i}-2 \sum_{j \neq i} \mathbf{M}_{i j} \eta_{j}<w_{i} .\end{cases}
$$

In this way, coordinate-wise minimization of the penalized quadratic form with respect to $\eta_{i}$ is achieved. Therefore, following the coordinate descent algorithm, by cycling $i=1,2, \ldots,(p+$ $q), 1,2, \ldots,(p+q) \ldots$ until all $\eta_{i}^{\prime} s$ converge, we obtain the minimizer, which is denoted as $\left(\boldsymbol{\beta}^{(1)}, \boldsymbol{d}^{(1)}\right)$. Afterwards, with $\left(\boldsymbol{\beta}^{(1)}, \boldsymbol{d}^{(1)}\right)$, we solve the minimum of the quadratic term regarding $\boldsymbol{\gamma}$, which is recored as $\gamma^{(1)}$. Consequently, $\tilde{\mathbf{D}}^{(1)}$ and $\tilde{\mathbf{T}}^{(1)}$ are updated by using $\boldsymbol{d}^{(1)}$ and $\boldsymbol{\gamma}^{(1)}$, and $\sigma^{2(1)}$ is updated by equation (4.7), then we obtain the new estimates of the whole parameter set, namely $\left(\boldsymbol{\beta}^{(1)}, \boldsymbol{d}^{(1)}, \boldsymbol{\gamma}^{(1)}, \sigma^{2(1)}\right)$.

\subsection{Asymptotic Results}

Recall the good properties of a model selection procedure, it is recommended that: 1 . the procedure identifies the true model with probability one; 2 . coefficient estimates are consistent; 3 . coefficient estimates converge to the true values with a certain rate. In this section, we illustrate that the proposed procedure possesses the above properties. 


\subsubsection{Notations and Assumptions}

Let $\Phi$ denote the whole estimator involving $(\boldsymbol{\beta}, \boldsymbol{d}, \boldsymbol{\gamma})$, and let the true parameters be $\Phi_{0}$. Splitting $\Phi_{0}$ into non-zero and zero sets, we denote $\Phi_{0}$ as $\Phi_{0}=\left(\Phi_{10}, \Phi_{20}\right)$, where $\Phi_{10}$ represents the set of true non-zero parameters and $\Phi_{20}$ represents the set of true zero parameters. More specifically, $\left(\Phi_{10}, \Phi_{20}\right)$ can be written as $\Phi_{10}=\left(\boldsymbol{\beta}_{10}, \boldsymbol{d}_{10}, \boldsymbol{\gamma}_{10}\right)$ and $\Phi_{20}=\left(\boldsymbol{\beta}_{20}, \boldsymbol{d}_{20}, \boldsymbol{\gamma}_{20}\right)$ in terms of $(\boldsymbol{\beta}, \boldsymbol{d}, \boldsymbol{\gamma})$, where $\Phi_{10}$ is an $s \times 1$ vector and $\Phi_{20}$ is a $t \times 1$ vector. In a similar manner, splitting $\Phi$ into $\left(\Phi_{1}, \Phi_{2}\right)$, let $\Phi_{1}=\left(\boldsymbol{\beta}_{1}, \boldsymbol{d}_{1}, \boldsymbol{\gamma}_{1}\right)$ represent the estimator of $\Phi_{10}$ and $\Phi_{2}=\left(\boldsymbol{\beta}_{2}, \boldsymbol{d}_{2}, \boldsymbol{\gamma}_{2}\right)$ represent the estimator of $\Phi_{20}$. For the design matrices, let $X_{(1)}=\left(X_{(1), 1}{ }^{\prime}, X_{(1), 2}{ }^{\prime}, \ldots, X_{(1), m}{ }^{\prime}\right)^{\prime}$ be the stacked fixed design matrix corresponding to $\boldsymbol{\beta}_{10}$ and $\tilde{V}_{(1)}=\operatorname{diag}\left(V_{(1), 1}, V_{(1), 2}, \ldots, V_{(1), m}\right)$ be the block diagonal random designed matrix corresponding to $\left(\boldsymbol{d}_{10}, \gamma_{10}\right)$. In addition, since we are considering the case when the sample size $m$ approaches infinity, there are some constraints required for the design matrices and log-likelihood, which are presented as follows:

1. $\max \left\{\left|x_{i j}\right|,\left|z_{i j}\right|\right\}<c_{x, z}<\infty$. All the elements in the design matrices are uniformly bounded by a constant.

2. $n_{i}<c_{n}<\infty$ for $i=1,2, \ldots, m$. Subgroup sizes are uniformly bounded by by anstant.

3. $L_{i}(\Phi) \in C_{B\left(\Phi_{0}, \delta\right)}^{(3)}$ and $\left|\frac{\partial^{3} L_{i}(\Phi)}{\partial \phi_{j} \partial \phi_{l} \partial \phi_{h}}\right|<R_{L}=O_{P}(1)$ for $i=1,2, \ldots, m$. The log-likelihood for all subjects are third order continuous differentiable in the neighborhood of $\Phi_{0}$, and uniformly bounded by a random variable $R_{L}$, where $R_{L}$ is bounded in probability.

4. Given that $\Phi_{20}=\mathbf{0}$, the fisher information $\mathrm{I}\left(\Phi_{10}\right)$ is finite and positive definite.

To obtain a consistent estimator of $\Phi_{0}$, it first requires the procedure to identify the sparse pattern with probability tending to one, namely $P\left(\Phi_{2} \rightarrow \Phi_{20}\right)=1$ as $m \rightarrow \infty$. To ensure the suggested limiting property is satisfied, we have the following theorem.

\subsubsection{Selection Consistency}

Theorem 1. Let $\lambda_{m} \rightarrow \infty$, when $\Phi_{1}$ is a $\sqrt{m}$ consistent estimator of $\Phi_{10}$, the penalized likelihood $Q(\Phi)$ defined in (4.1) is maximized when $\Phi_{2}=0$ with probability tending to 1 , where 
$\Phi=\left(\Phi_{1}, \Phi_{2}\right)$

Proof. Let $\phi_{j}$ be the $j$ th element of $\Phi$. In order to show $Q(\Phi)$ reaches a local maximum when all $\phi_{j}{ }^{\prime} s$ in the set of $\Phi_{2}$ are zero with probability tending to 1 , it suffices to detect the partial derivative behavior in the neighbourhood of $\Phi_{20}$. Furthermore, recall that only $(\boldsymbol{\beta}, \boldsymbol{d})$ can fully decide the sparsity, we apply Taylor expansion for the partial derivative $\frac{\partial}{\partial \phi_{j}} Q(\Phi)$ for all $\phi_{j} \in\left(\boldsymbol{\beta}_{2}, \boldsymbol{d}_{2}\right)$, which takes the form:

$$
\begin{aligned}
\frac{\partial}{\partial \phi_{j}} Q(\Phi)= & \frac{\partial}{\partial \phi_{j}} L\left(\Phi_{0}\right)-\sum_{l=1}^{s+t} \frac{\partial^{2}}{\partial \phi_{j} \partial \phi_{l}} L\left(\Phi_{0}\right)\left(\phi_{l}-\phi_{l 0}\right) \\
& +\frac{1}{2} \sum_{l=1}^{s+t} \sum_{h=1}^{s+t} \frac{\partial^{3}}{\partial \phi_{j} \partial \phi_{l} \partial \phi_{h}} L\left(\Phi_{*}\right)\left(\phi_{l}-\phi_{l 0}\right)\left(\phi_{h}-\phi_{h 0}\right)-\lambda_{m} \frac{1}{\hat{\phi}_{j}} \operatorname{sgn}\left(\phi_{j}\right),
\end{aligned}
$$

where $\left(\phi_{l 0}, \phi_{h 0}\right)$ are the $l$ th, $h$ th elements of $\Phi_{0}, \hat{\phi}_{j}$ either takes the value of $\hat{\beta}_{j}$ or $\hat{d}_{j}$ and $\Phi_{*}$ is the value between $\Phi$ and $\Phi_{0}$.

More specifically, the Taylor expansion of the partial derivative of the $i$ th subject is given by

$$
\begin{aligned}
\frac{\partial}{\partial \phi_{j}} L_{i}(\Phi)= & \frac{\partial}{\partial \phi_{j}} L_{i}\left(\Phi_{0}\right)-\sum_{l=1}^{s+t} \frac{\partial^{2}}{\partial \phi_{j} \partial \phi_{l}} L_{i}\left(\Phi_{0}\right)\left(\phi_{l}-\phi_{l 0}\right) \\
& +\frac{1}{2} \sum_{l=1}^{s+t} \sum_{h=1}^{s+t} \frac{\partial^{3}}{\partial \phi_{j} \partial \phi_{l} \partial \phi_{h}} L_{i}\left(\Phi_{*}\right)\left(\phi_{l}-\phi_{l 0}\right)\left(\phi_{h}-\phi_{h 0}\right) .
\end{aligned}
$$

When $\phi_{j}$ takes the form of $\beta_{j}$, the partial derivative is given by

$$
m^{-\frac{1}{2}} \frac{\partial}{\partial \beta_{j}} L_{i}\left(\Phi_{0}\right)=m^{-\frac{1}{2}} X_{i, \bullet j}^{\prime} V_{(0), i}^{-1}\left(y_{i}-X_{i} \boldsymbol{\beta}_{0}\right)
$$

where $\boldsymbol{\beta}_{0}=\left(\beta_{10}, \beta_{20}\right), X_{i, \bullet j}$ is $j$ th column of $X_{i}$ and $V_{(0), i}$ is the $V_{i}$ evaluated at $\Phi_{0}$. When $\phi_{j}$ takes the form of $d_{j}$, the partial derivative is given by

$$
m^{-\frac{1}{2}} \frac{\partial}{\partial d_{j}} L_{i}\left(\Phi_{0}\right)=\frac{1}{2} m^{-\frac{1}{2}}\left[-\operatorname{Tr}\left(V_{(0), i}^{-1} \frac{\partial V_{(0), i}}{\partial d_{j}}\right)+\left(y_{i}-X_{i} \boldsymbol{\beta}_{0}\right)^{\prime}\left(V_{(0), i}^{-1} \frac{\partial V_{(0), i}}{\partial d_{j}} V_{(0), i}^{-1}\right)\left(y_{i}-X_{i} \boldsymbol{\beta}_{0}\right)\right]
$$


Firstly, note that $\frac{\partial V_{(0), i}}{\partial d_{j}}$ is a zero matrix, then $m^{-\frac{1}{2}} \frac{\partial}{\partial d_{j}} L_{i}\left(\Phi_{0}\right)$ in (4.13) is zero. Secondly, expectation and variance of (4.12) are given by

$$
\begin{aligned}
E\left[m^{-\frac{1}{2}} \frac{\partial}{\partial \beta_{j}} L_{i}\left(\Phi_{0}\right)\right] & =m^{-\frac{1}{2}} X_{i, \bullet j}^{\prime} V_{(0), i}^{-1} E\left(y_{i}-X_{i} \boldsymbol{\beta}_{0}\right)=0 \\
\operatorname{Var}\left[m^{-\frac{1}{2}} \frac{\partial}{\partial \beta_{j}} L_{i}\left(\Phi_{0}\right)\right] & =m^{-1} X_{i, \bullet j}^{\prime} V_{(0), i}^{-1} X_{i, \bullet j}
\end{aligned}
$$

Recall condition 1, every element in $X_{i, \bullet j}^{\prime}$ and $V_{(0), i}^{-1}$ is bounded, thus $X_{i, \bullet j}^{\prime} V_{(0), i}^{-1} X_{i, \bullet j}<C_{(4.12)}<$ $\infty$. By the Chebyshev's inequality, we have

$$
P\left\{m^{-\frac{1}{2}} \frac{\partial}{\partial \beta_{j}} L_{i}\left(\Phi_{0}\right)>\epsilon_{0}\right\}<\frac{C_{(4.12)}}{m \epsilon_{0}^{2}}
$$

Therefore $m^{-\frac{1}{2}} \frac{\partial}{\partial \beta_{j}} L\left(\Phi_{0}\right)$ takes the form:

$$
m^{-\frac{1}{2}} \frac{\partial}{\partial \beta_{j}} L_{i}\left(\Phi_{0}\right)=O_{p}\left(m^{-1}\right)
$$

Since $C_{(4.12)}$ is a constant regardless of $i$, we can take sum of all the partial derivatives from 1 to $m$ and have the following results:

$$
\begin{aligned}
& \frac{1}{\sqrt{m}} \frac{\partial}{\partial \beta_{j}} L\left(\Phi_{0}\right)=\frac{1}{\sqrt{m}} \sum_{i=1}^{m} \frac{\partial}{\partial \beta_{j}} L_{i}\left(\Phi_{0}\right)=O_{p}(1) \\
& \frac{1}{\sqrt{m}} \frac{\partial}{\partial d_{j}} L\left(\Phi_{0}\right)=\frac{1}{\sqrt{m}} \sum_{i=1}^{m} \frac{\partial}{\partial d_{j}} L_{i}\left(\Phi_{0}\right)=0
\end{aligned}
$$

which completes the analysis of the 1st order partial derivative. Now considering the second order partial derivative, it possesses the following property:

$$
\left.\frac{1}{m} \nabla^{2} L(\Phi)\right|_{\Phi=\Phi_{0}} \rightarrow_{p} E\left(\left.\nabla^{2} L((\Phi))\right|_{\Phi=\Phi_{0}}\right.
$$

where $E\left(\left.\nabla^{2} L((\Phi))\right|_{\Phi=\Phi_{0}}\right.$ is the negative fisher information evaluated at $\Phi_{0}$. More specifically, 
$E\left(\nabla^{2} L((\Phi))\right.$ takes the form:

$$
\begin{aligned}
E\left[\frac{\partial^{2}}{\partial \beta_{j} \partial \beta_{l}} L\left(\Phi_{0}\right)\right]= & -E\left[X_{\bullet j}^{\prime} \tilde{V}_{(0)}^{-1} X_{\bullet l}\right] \\
E\left[\frac{\partial^{2}}{\partial \beta_{j} \partial d_{l}} L\left(\Phi_{0}\right)\right]= & -E\left[X_{\bullet j}^{\prime}\left(\tilde{V}_{(0)}^{-1} \frac{\partial \tilde{V}_{(0)}}{\partial d_{l}} \tilde{V}_{(0)}^{-1}\right)\left(y-X \beta_{0}\right)\right] \\
E\left[\frac{\partial^{2}}{\partial \beta_{j} \partial \gamma_{l}} L\left(\Phi_{0}\right)\right]= & -E\left[X_{\bullet j}^{\prime}\left(\tilde{V}_{(0)}^{-1} \frac{\partial \tilde{V}_{(0)}}{\partial \gamma_{l}} \tilde{V}_{(0)}^{-1}\right)\left(y-X \beta_{0}\right)\right] \\
E\left[\frac{\partial^{2}}{\partial d_{j} \partial d_{l}} L\left(\Phi_{0}\right)\right]= & -\frac{1}{2} \operatorname{tr}\left(\tilde{V}_{(0)}^{-1} \frac{\partial^{2} \tilde{V}_{(0)}}{\partial d_{j} d_{l}}-\tilde{V}_{(0)}^{-1} \frac{\partial \tilde{V}_{(0)}}{\partial d_{j}} \tilde{V}_{(0)}^{-1} \frac{\partial \tilde{V}_{(0)}}{\partial d_{l}}\right) \\
& -\frac{1}{2} \operatorname{tr}\left(\tilde{V}_{(0)}^{-1} \frac{\partial \tilde{V}_{(0)}}{\partial d_{j}} \tilde{V}_{(0)}^{-1} \frac{\partial \tilde{V}_{(0)}}{\partial d_{l}}-\tilde{V}_{(0)}^{-1} \frac{\partial^{2} \tilde{V}_{(0)}}{\partial d_{j} d_{l}}+\tilde{V}_{(0)}^{-1} \frac{\partial \tilde{V}_{(0)}}{\partial d_{l}} \tilde{V}_{(0)}^{-1} \frac{\partial \tilde{V}_{(0)}}{\partial d_{j}}\right) \\
= & -\frac{1}{2} \operatorname{tr}\left(\tilde{V}_{(0)}^{-1} \frac{\partial \tilde{V}_{(0)}}{\partial d_{l}} \tilde{V}_{(0)}^{-1} \frac{\partial \tilde{V}_{(0)}}{\partial d_{j}}\right) \\
E\left[\frac{\partial^{2}}{\partial d_{j} \partial \gamma_{l}} L\left(\Phi_{0}\right)\right]= & -\frac{1}{2} \operatorname{tr}\left(\tilde{V}_{(0)}^{-1} \frac{\partial^{2} \tilde{V}_{(0)}}{\partial d_{j} \gamma_{l}}-\tilde{V}_{(0)}^{-1} \frac{\partial \tilde{V}_{(0)}}{\partial d_{j}} \tilde{V}_{(0)}^{-1} \frac{\partial \tilde{V}_{(0)}}{\partial \gamma_{l}}\right) \\
& -\frac{1}{2} \operatorname{tr}\left(\tilde{V}_{(0)}^{-1} \frac{\partial \tilde{V}_{(0)}}{\partial d_{j}} \tilde{V}_{(0)}^{-1} \frac{\partial \tilde{V}_{(0)}}{\partial \gamma_{l}}-\tilde{V}_{(0)}^{-1} \frac{\partial^{2} \tilde{V}_{(0)}}{\partial d_{j} \gamma_{l}}+\tilde{V}_{(0)}^{-1} \frac{\partial \tilde{V}_{(0)}}{\partial \gamma_{l}} \tilde{V}_{(0)}^{-1} \frac{\partial \tilde{V}_{(0)}}{\partial d_{j}}\right) \\
= & -\frac{1}{2} \operatorname{tr}\left(\tilde{V}_{(0)}^{-1} \frac{\partial \tilde{V}_{(0)}}{\partial \gamma_{l}} \tilde{V}_{(0)}^{-1} \frac{\partial \tilde{V}_{(0)}}{\partial d_{j}}\right)
\end{aligned}
$$

In particular, $\frac{\partial^{2}}{\partial \beta_{j} \partial \beta_{l}} L\left(\Phi_{0}\right)$ is a term regardless of $y$. Hence with condition $1,\left|\frac{\partial^{2}}{\partial \beta_{j} \partial \beta_{l}} L\left(\Phi_{0}\right)\right| \leq$ $C m$, where $C$ is a constant. In addition, since $\frac{\partial \tilde{V}}{\partial \phi_{j}}$ are zero matrices when $\Phi$ takes the value $\Phi_{0}$, the expectations of all the 2 nd order partial derivatives above are zero (except the 1 st expectation). Consequently, for the 2nd expectation, it follows:

$$
\frac{1}{m} \frac{\partial^{2}}{\partial \beta_{j} \partial d_{l}} L\left(\Phi_{0}\right) \rightarrow_{p} E\left[\frac{\partial^{2}}{\partial \beta_{j} d_{l}} L\left(\Phi_{0}\right)\right]=0
$$


Similarly, we have the results:

$$
\begin{aligned}
& \frac{1}{m} \frac{\partial^{2}}{\partial \beta_{j} \partial \gamma_{l}} L\left(\Phi_{0}\right) \rightarrow_{p} 0, \\
& \frac{1}{m} \frac{\partial^{2}}{\partial d_{j} \partial d_{l}} L\left(\Phi_{0}\right) \rightarrow_{p} 0, \\
& \frac{1}{m} \frac{\partial^{2}}{\partial d_{j} \partial \gamma_{l}} L\left(\Phi_{0}\right) \rightarrow_{p} 0 .
\end{aligned}
$$

Following the above analysis, $\frac{1}{\sqrt{m}} \frac{\partial}{\partial \beta_{j}} Q(\Phi)$ is given by

$$
\begin{aligned}
\frac{1}{\sqrt{m}} \frac{\partial}{\partial \beta_{j}} Q(\Phi)= & \frac{1}{\sqrt{m}}\left[\frac{\partial L\left(\Phi_{0}\right)}{\partial \beta_{j}}-\sum_{l=1}^{n_{\beta}} \frac{\partial^{2} L\left(\Phi_{0}\right)}{\partial \beta_{j} \partial \beta_{l}}\left(\beta_{l}-\beta_{l 0}\right)\right. \\
& -\sum_{l=1}^{n_{d}} \frac{\partial^{2} L\left(\Phi_{0}\right)}{\partial \beta_{j} \partial d_{l}}\left(d_{l}-d_{l 0}\right)-\sum_{l=1}^{n_{\gamma}} \frac{\partial^{2} L\left(\Phi_{0}\right)}{\partial \beta_{j} \partial \gamma_{l}}\left(\gamma_{l}-\gamma_{l 0}\right) \\
& +\frac{1}{2} \sum_{i=1}^{m} \sum_{l=1}^{n_{\beta}} \sum_{h=1}^{n_{\beta}} \frac{\partial^{3} L_{i}\left(\Phi_{*}\right)}{\partial \beta_{j} \partial \beta_{l} \partial \beta_{h}}\left(\beta_{l}-\beta_{l 0}\right)\left(\beta_{h}-\beta_{h 0}\right) \\
& +\frac{1}{2} \sum_{i=1}^{m} \sum_{l=1}^{n_{d}} \sum_{h=1}^{n_{d}} \frac{\partial^{3} L_{i}\left(\Phi_{*}\right)}{\partial \beta_{j} \partial d_{l} \partial d_{h}}\left(d_{l}-d_{l 0}\right)\left(d_{h}-d_{h 0}\right) \\
& +\frac{1}{2} \sum_{i=1}^{m} \sum_{l=1}^{n_{\gamma}} \sum_{h=1}^{n_{\gamma}} \frac{\partial^{3} L_{i}\left(\Phi_{*}\right)}{\partial \beta_{j} \partial \gamma_{l} \partial \gamma_{h}}\left(\gamma_{l}-\gamma_{l 0}\right)\left(\gamma_{h}-\gamma_{h 0}\right) \\
& +\sum_{i=1}^{m} \sum_{l=1}^{n_{\beta}} \sum_{h=1}^{n_{d}} \frac{\partial^{3} L_{i}\left(\Phi_{*}\right)}{\partial \beta_{j} \partial \beta_{l} \partial d_{h}}\left(\beta_{l}-\beta_{l 0}\right)\left(d_{h}-d_{h 0}\right) \\
& +\sum_{i=1}^{m} \sum_{l=1}^{n_{\beta}} \sum_{h=1}^{n_{\gamma}} \frac{\partial^{3} L_{i}\left(\Phi_{*}\right)}{\partial \beta_{j} \partial \beta_{l} \partial \gamma_{h}}\left(\beta_{l}-\beta_{l 0}\right)\left(\gamma_{h}-\gamma_{h 0}\right) \\
& \left.+\sum_{i=1}^{m} \sum_{l=1}^{n_{d}} \sum_{h=1}^{n_{\gamma}} \frac{\partial^{3} L_{i}\left(\Phi_{*}\right)}{\partial \beta_{j} \partial d_{l} \partial \gamma_{h}}\left(d_{l}-d_{l 0}\right)\left(\gamma_{h}-\gamma_{h 0}\right)-\lambda_{m} \frac{1}{\hat{\beta}_{j}} \operatorname{sgn}\left(\beta_{j}\right)\right]
\end{aligned}
$$

where $\Phi_{*}$ is between $\Phi_{0}$ and $\Phi$, and $\left(n_{\beta}, n_{d}, n_{\gamma}\right)$ are the vectors' lengths corresponding to $\boldsymbol{\beta}, \boldsymbol{d}$ and $\gamma$. When $\Phi$ is a $\sqrt{m}$ consistent estimator of $\Phi_{0}$, it follows:

$$
P\left(\left|\Phi_{*}-\Phi_{0}\right|<\frac{C}{\sqrt{m}}\right)>P\left(\left|\Phi-\Phi_{0}\right|<\frac{C}{\sqrt{m}}\right)>1-\epsilon,
$$


thus $\left|\Phi_{*}-\Phi_{0}\right|=O_{p}\left(m^{-\frac{1}{2}}\right)$. With condition 3, the 3rd order partial derivatives are bounded in probability, and $\frac{1}{\sqrt{m}} \frac{\partial}{\partial \beta_{j}} Q(\phi)$ can be justified as

$$
\frac{1}{\sqrt{m}} \frac{\partial}{\partial \beta_{j}} Q(\Phi)=\frac{\lambda_{m}}{\sqrt{m}} \frac{1}{\hat{\beta}_{j}} \operatorname{sgn}\left(\beta_{j}\right)+O_{p}(1)
$$

When $\beta_{j 0}=0$, the $j$ th element of $\sqrt{m}$ consistent estimator $\hat{\beta}_{j}=O_{p}\left(m^{-\frac{1}{2}}\right)$, then $\left|\sqrt{m} \hat{\beta}_{j}\right|=O_{p}(1)$. As a result, when $\lambda_{m} \rightarrow \infty$, the sign of the derivative is determined by the sign of $\beta_{j}$, and this completes the analysis when $\phi_{j}=\beta_{j}$.

Next, considering the case when $\phi_{j}=d_{j}$ and following the notations in the previous analysis, the partial derivative takes the form:

$$
\begin{aligned}
\frac{1}{\sqrt{m}} \frac{\partial}{\partial d_{j}} Q(\Phi)= & \frac{1}{\sqrt{m}}\left[\frac{\partial L\left(\Phi_{0}\right)}{\partial d_{j}}-\sum_{l=1}^{n_{\beta}} \frac{\partial^{2} L\left(\Phi_{0}\right)}{\partial d_{j} \partial \beta_{l}}\left(\beta_{l}-\beta_{l 0}\right)\right. \\
& -\sum_{l=1}^{n_{d}} \frac{\partial^{2} L\left(\Phi_{0}\right)}{\partial d_{j} \partial d_{l}}\left(d_{l}-d_{l 0}\right)-\sum_{l=1}^{n_{\gamma}} \frac{\partial^{2} L\left(\Phi_{0}\right)}{\partial d_{j} \partial \gamma_{l}}\left(\gamma_{l}-\gamma_{l 0}\right) \\
& +\frac{1}{2} \sum_{i=1}^{m} \sum_{l=1}^{n_{\beta}} \sum_{h=1}^{n_{\beta}} \frac{\partial^{3} L_{i}\left(\Phi_{*}\right)}{\partial d_{j} \partial \beta_{l} \partial \beta_{h}}\left(\beta_{l}-\beta_{l 0}\right)\left(\beta_{h}-\beta_{h 0}\right) \\
& +\frac{1}{2} \sum_{i=1}^{m} \sum_{l=1}^{n_{d}} \sum_{h=1}^{n_{d}} \frac{\partial^{3} L_{i}\left(\Phi_{*}\right)}{\partial d_{j} \partial d_{l} \partial d_{h}}\left(d_{l}-d_{l 0}\right)\left(d_{h}-d_{h 0}\right) \\
& +\frac{1}{2} \sum_{i=1}^{m} \sum_{l=1}^{n_{\gamma}} \sum_{h=1}^{n_{\gamma}} \frac{\partial^{3} L_{i}\left(\Phi_{*}\right)}{\partial d_{j} \partial \gamma_{l} \partial \gamma_{h}}\left(\gamma_{l}-\gamma_{l 0}\right)\left(\gamma_{h}-\gamma_{h 0}\right) \\
& +\sum_{i=1}^{m} \sum_{l=1}^{n_{\beta}} \sum_{h=1}^{n_{d}} \frac{\partial^{3} L_{i}\left(\Phi_{*}\right)}{\partial d_{j} \partial \beta_{l} \partial d_{h}}\left(\beta_{l}-\beta_{l 0}\right)\left(d_{h}-d_{h 0}\right) \\
& +\sum_{i=1}^{m} \sum_{l=1}^{n_{\beta}} \sum_{h=1}^{n_{\gamma}} \frac{\partial^{3} L_{i}\left(\Phi_{*}\right)}{\partial d_{j} \partial \beta_{l} \partial \gamma_{h}}\left(\beta_{l}-\beta_{l 0}\right)\left(\gamma_{h}-\gamma_{h 0}\right) \\
& \left.+\sum_{i=1}^{m} \sum_{l=1}^{n_{d}} \sum_{h=1}^{n_{\gamma}} \frac{\partial^{3} L_{i}\left(\Phi_{*}\right)}{\partial d_{j} \partial d_{l} \partial \gamma_{h}}\left(d_{l}-d_{l 0}\right)\left(\gamma_{h}-\gamma_{h 0}\right)-\lambda_{m} \frac{1}{\hat{\beta}_{j}} \operatorname{sgn}\left(\beta_{j}\right)\right] .
\end{aligned}
$$

Similarly, the partial derivative can be expressed as

$$
\frac{1}{\sqrt{m}} \frac{\partial}{\partial d_{j}} Q(\Phi)=-\lambda_{m} \frac{1}{\sqrt{m}} \frac{1}{\hat{d}_{j}} \operatorname{sgn}\left(d_{j}\right)+O_{p}(1)
$$


When $d_{j 0}=0$ and $\hat{d}_{j}$ is the $j$ th element of a $\sqrt{m}$ consistent estimator corresponding to $d_{j 0}, \hat{d}_{j}$ takes the form: $\hat{d}_{j}=O_{p}\left(m^{-\frac{1}{2}}\right)$, then $\sqrt{m} \hat{d}_{j}=O_{p}(1)$. Consequently, when $\lambda_{m} \rightarrow \infty$, the sign is determined by $\operatorname{sgn}\left(d_{j}\right)$.

To summarize the results, the derivative is negative when $\phi_{j}>0$ and positive when $\phi_{j}<0$ with probability tending to 1 , hence the local maximum could be obtained when $\Phi_{2}=0$ with probability tending to 1 .

\subsubsection{Estimation Consistency}

Last theorem demonstrates that the true zero set can be identified with probability tending to 1 by maximizing the $Q(\Phi)$ with a $\sqrt{m}$ consistent estimator of $\Phi_{10}$, and the subsequent theorem is founded to prove that there exists a $\sqrt{m}$ consistent estimator of $\Phi_{10}$.

Theorem 2. Given that $\Phi_{20}=0$ and $\frac{\lambda_{m}}{\sqrt{m}} \rightarrow 0$, there exists a local maximizer $\left(\hat{\Phi}_{1}, 0\right)$ of the function (4.1), such that $\hat{\Phi}_{1}$ is $\sqrt{m}$ consistent of $\Phi_{10}$.

Proof. Let $\Phi_{1}=\Phi_{10}+\alpha_{m} \mu$, where $\alpha_{m}=\frac{1}{\sqrt{m}}$ and $\|\mu\|<C$ for a constant $C$. By using the Taylor expansion for the $i$ th log-likelihood, the difference takes the form:

$$
\begin{aligned}
D_{i}(\mu) & =L_{i}\left(\Phi_{1}\right)-L_{i}\left(\Phi_{10}\right) \\
& =\left\{L_{i}\left(\Phi_{10}+\alpha_{m} \mu\right)-L_{i}\left(\Phi_{10}\right)\right\} \\
& =\alpha_{m}\left(\nabla L_{i}\left(\Phi_{10}\right)\right)^{\prime} \mu+\frac{1}{2} \mu^{\prime}\left[\nabla^{2} L_{i}\left(\Phi_{10}\right)\right] \mu \alpha_{m}{ }^{2}+\frac{1}{6} \sum_{j=1}^{s} \sum_{l=1}^{s} \sum_{h=1}^{s} \frac{\partial^{3}}{\partial \phi_{j} \partial \phi_{l} \partial \phi_{h}} L_{i}\left(\Phi^{*}\right) \alpha_{m}{ }^{3} \mu_{j} \mu_{l} \mu_{h}
\end{aligned}
$$

where $\Phi^{*}$ is a value between $\Phi_{1}$ and $\Phi_{10}$, and $\mu_{j}, \mu_{l}, \mu_{k}$ are the projection lengths of $\mu$ to $\phi_{j}, \phi_{l}, \phi_{k}$. With condition $3, \frac{\partial^{3}}{\partial \phi_{j} \partial \phi_{l} \partial \phi_{k}} L_{i}\left(\Phi^{*}\right)$ is bounded in probability. Therefore, the remainder has the form:

$$
\frac{1}{6} \sum_{j=1}^{r} \sum_{l=1}^{r} \sum_{k=1}^{r} \frac{\partial^{3}}{\partial \phi_{j} \partial \phi_{l} \partial \phi_{k}} L_{i}\left(\Phi^{*}\right) \alpha_{m}{ }^{3} \mu_{j} \mu_{l} \mu_{k}=O_{P}\left(m^{-\frac{3}{2}}\right) .
$$


In this way, $D_{i}(\mu)$ can be also expressed as

$$
D_{i}(\mu)=\frac{1}{\sqrt{m}}\left(\nabla L_{i}\left(\Phi_{10}\right)\right)^{\prime} \mu+\frac{1}{2 m} \mu^{\prime}\left[\nabla^{2} L_{i}\left(\Phi_{10}\right)\right] \mu+O_{P}\left(m^{-\frac{3}{2}}\right),
$$

Now considering the gradient $\nabla L_{i}\left(\Phi_{10}\right)$, the partial derivatives of $L_{i}\left(\Phi_{10}\right)$ corresponding to $\beta_{j}, d_{j}, \gamma_{j}$ are given by

$$
\begin{gathered}
m^{-\frac{1}{2}} \frac{\partial}{\partial \beta_{j}} L_{i}\left(\Phi_{1}\right)=m^{-\frac{1}{2}} X_{(1), i, \boldsymbol{\bullet}}^{\prime} V_{(1), i}^{-1}\left(y_{i}-X_{(1), i} \boldsymbol{\beta}_{1}\right), \\
m^{-\frac{1}{2}} \frac{\partial}{\partial d_{j}} L_{i}\left(\Phi_{1}\right)=\frac{1}{2} m^{-\frac{1}{2}}\left[-\operatorname{Tr}\left(V_{(1), i}^{-1} \frac{\partial V_{(1), i}}{\partial d_{j}}\right)+\left(y_{i}-X_{(1), i} \boldsymbol{\beta}_{1}\right)^{\prime}\left(V_{(1), i}^{-1} \frac{\partial V_{(1), i}}{\partial d_{j}} V_{(1), i}^{-1}\right)\left(y_{i}-X_{(1), i} \boldsymbol{\beta}_{1}\right)\right]
\end{gathered}
$$

and

$$
m^{-\frac{1}{2}} \frac{\partial}{\partial \gamma_{j}} L_{i}\left(\Phi_{1}\right)=\frac{1}{2} m^{-\frac{1}{2}}\left[-\operatorname{Tr}\left(V_{(1), i}^{-1} \frac{\partial V_{(1), i}}{\partial \gamma_{j}}\right)+\left(y_{i}-X_{(1), i} \boldsymbol{\beta}_{1}\right)^{\prime}\left(V_{(1), i}^{-1} \frac{\partial V_{(1), i}}{\partial \gamma_{j}} V_{(1), i}^{-1}\right)\left(y_{i}-X_{(1), i} \boldsymbol{\beta}_{1}\right)\right] .
$$

Specifically, in equation (4.15), $X_{(1), i, \bullet j}^{\prime}$ represents the the $j$ column of matrix $X_{(1), i}$. Next, we analyze the partial derivatives from (4.15) to (4.17), where the expectation and variance of the first equation are given by

$$
\begin{aligned}
E\left[m^{-\frac{1}{2}} \frac{\partial}{\partial \beta_{j}} L_{i}\left(\Phi_{1}\right)\right] & =m^{-\frac{1}{2}} X_{(1), i \bullet \bullet}^{\prime} V_{(1), i}^{-1} E\left(y-X_{(1), i} \boldsymbol{\beta}_{1}\right)=0 \\
\operatorname{Var}\left[m^{-\frac{1}{2}} \frac{\partial}{\partial \beta_{j}} L_{i}\left(\Phi_{1}\right)\right] & =m^{-1} X_{(1), i, \bullet j}^{\prime} V_{(1), i}^{-1} X_{(1), i, \bullet j}
\end{aligned}
$$

With condition 1, every element in $X_{(1), i, \bullet j}^{\prime}$ and $V_{(1), i}^{-1}$ is bounded, therefore $X_{(1), i, \bullet j}^{\prime} V_{(1), i}^{-1} X_{(1), i, \mathfrak{\bullet}}<$ $C_{(4.15)}<\infty$. By the Chebyshev's inequality, we have

$$
P\left\{m^{-\frac{1}{2}} \frac{\partial}{\partial \beta_{j}} L_{i}\left(\Phi_{1}\right)>\epsilon_{1}\right\}<\frac{C_{(4.15)}}{m \epsilon_{1}^{2}} .
$$


Thus, $m^{-\frac{1}{2}} \frac{\partial}{\partial \beta_{j}} L_{i}\left(\Phi_{1}\right)=O_{p}\left(m^{-1}\right)$. Similarly, for equations (4.16) and (4.17), we have the results:

$$
\begin{aligned}
E\left[m^{-\frac{1}{2}} \frac{\partial}{\partial d_{j}} L_{i}\left(\Phi_{1}\right)\right] & =m^{-\frac{1}{2}}\left[-\operatorname{Tr}\left(V_{(1), i}^{-1} \frac{\partial V_{(1), i}}{\partial d_{j}}\right)+\operatorname{Tr}\left(V_{(1), i}^{-1} \frac{\partial V_{(1), i}}{\partial d_{j}} V_{(1), i}^{-1} V_{(1), i}\right)\right]=0 \\
\operatorname{Var}\left[m^{-\frac{1}{2}} \frac{\partial}{\partial d_{j}} L_{i}\left(\Phi_{1}\right)\right] & =m^{-1} \operatorname{Tr}\left[V_{(1), i}^{-1} \frac{\partial V_{(1), i}}{\partial d_{j}} V_{(1), i}^{-1} \frac{\partial V_{(1), i}}{\partial d_{j}}\right]
\end{aligned}
$$

and

$$
\begin{aligned}
E\left[m^{-\frac{1}{2}} \frac{\partial}{\partial \gamma_{j}} L_{i}\left(\Phi_{1}\right)\right] & =m^{-\frac{1}{2}}\left[-\operatorname{Tr}\left(V_{(1), i}^{-1} \frac{\partial V_{(1), i}}{\partial \gamma_{j}}\right)+\operatorname{Tr}\left(V_{(1), i}^{-1} \frac{\partial V_{(1), i}}{\partial \gamma_{j}} V_{(1), i}^{-1} V_{(1), i}\right)\right]=0 \\
\operatorname{Var}\left[m^{-\frac{1}{2}} \frac{\partial}{\partial \gamma_{j}} L_{i}\left(\Phi_{1}\right)\right] & =m^{-1} \operatorname{Tr}\left[V_{(1), i}^{-1} \frac{\partial V_{(1), i}}{\partial \gamma_{j}} V_{(1), i}^{-1} \frac{\partial V_{(1), i}}{\partial \gamma_{j}}\right] .
\end{aligned}
$$

Again with condition 1, every element in $\frac{\partial V_{(1), i}}{\partial d_{j}}$ and $\frac{\partial V_{(1), i}}{\partial \gamma_{j}}$ is finite. Consequently, the traces in (4.19) and (4.20) must satisfy

$$
\begin{aligned}
& \operatorname{Tr}\left[V_{(1), i}^{-1} \frac{\partial V_{(1), i}}{\partial d_{j}} V_{(1), i}^{-1} \frac{\partial V_{(1), i}}{\partial d_{j}}\right]<C_{(4.16)}, \\
& \operatorname{Tr}\left[V_{(1), i}^{-1} \frac{\partial V_{(1), i}}{\partial \gamma_{j}} V_{(1), i}^{-1} \frac{\partial V_{(1), i}}{\partial \gamma_{j}}\right]<C_{(4.17)} .
\end{aligned}
$$

By using Chebyshev's inequality for (4.16) and (4.17), we have the results

$$
\begin{aligned}
& P\left\{m^{-\frac{1}{2}} \frac{\partial}{\partial d_{j}} L_{i}\left(\Phi_{1}\right)>\epsilon_{2}\right\}<\frac{C_{(4.16)}}{m \epsilon_{2}^{2}}, \\
& P\left\{m^{-\frac{1}{2}} \frac{\partial}{\partial \gamma_{j}} L_{i}\left(\Phi_{1}\right)>\epsilon_{3}\right\}<\frac{C_{(4.17)}}{m \epsilon_{3}^{2}} .
\end{aligned}
$$

To sum up, $m^{-\frac{1}{2}} \frac{\partial}{\partial \beta_{j}} L_{i}\left(\Phi_{1}\right)=O_{p}\left(m^{-1}\right), m^{-\frac{1}{2}} \frac{\partial}{\partial d_{j}} L_{i}\left(\Phi_{1}\right)=O_{p}\left(m^{-1}\right)$ and $m^{-\frac{1}{2}} \frac{\partial}{\partial \gamma_{j}} L_{i}\left(\Phi_{1}\right)=$ $O_{p}\left(m^{-1}\right)$. Therefore, $D_{i}(\mu)$ can be expressed as

$$
D_{i}(\mu)=O_{p}\left(m^{-1}\right) \mu+\frac{1}{2 m} \mu^{\prime}\left[\nabla^{2} L_{i}\left(\Phi_{10}\right)\right] \mu+O_{P}\left(m^{-\frac{3}{2}}\right) .
$$

Finally, note that $C_{(4.15)}, C_{(4.16)}$, and $C_{(4.17)}$ are constants regardless of $i$, we may replace them with a uniform bound $C_{\max }=\max \left(C_{(4.15)}, C_{(4.16)}, C_{(4.17)}\right)$, and the difference regarding the 
whole data can be expressed as

$$
\begin{aligned}
Q\left(\Phi_{1}\right)-Q\left(\Phi_{10}\right) & =L\left(\Phi_{1}\right)-L\left(\Phi_{10}\right)-\lambda_{m}\left[\sum_{j=1}^{s} \frac{1}{\hat{\phi}_{j}}\left(\left|\phi_{j 0}+\alpha_{m} u_{j}\right|-\left|\phi_{j 0}\right|\right)\right] \\
& =\sum_{i=1}^{m} L_{i}\left(\phi_{1}\right)-\sum_{i=1}^{m} L_{i}\left(\phi_{10}\right)-\lambda_{m} \sum_{j=1}^{s} \frac{1}{\hat{\phi}_{j}} \operatorname{sgn}\left(\phi_{j 0}\right) \alpha_{m} \mu_{j} \\
& =\sum_{i=1}^{m} D_{i}(\mu)-\frac{\lambda_{m}}{\sqrt{m}} \sum_{j=1}^{s} \frac{1}{\hat{\phi}_{j}} \operatorname{sgn}\left(\phi_{j 0}\right) \mu_{j} \\
& =O_{p}(1) \mu+\frac{1}{2 m} \mu^{\prime}\left[\nabla^{2} L\left(\Phi_{10}\right)\right] \mu+O_{P}\left(m^{-\frac{1}{2}}\right)-\frac{\lambda_{m}}{\sqrt{m}} \sum_{j=1}^{s} \frac{1}{\hat{\phi}_{j}} \operatorname{sgn}\left(\phi_{j 0}\right) \mu_{j} .
\end{aligned}
$$

Note that $\frac{\lambda_{m}}{\sqrt{m}} \sum_{j=1}^{s} \frac{1}{\hat{\phi}_{j}} \operatorname{sgn}\left(\phi_{j 0}\right) \mu_{j} \rightarrow_{p} \frac{\lambda_{m}}{\sqrt{m}} \sum_{j=1}^{s} \frac{1}{\phi_{j 0}} \operatorname{sgn}\left(\phi_{j 0}\right) \mu_{j} \rightarrow_{p} 0$ when $\left|\mu_{j}\right| \leq\|\mu\|<C$ and $\frac{\lambda_{m}}{\sqrt{m}} \rightarrow 0$. Additionally, the middle matrix of the quadratic term has the result $\frac{1}{m} \nabla^{2} L\left(\Phi_{10}\right) \rightarrow_{p}$ $-I\left(\Phi_{10}\right)$. Recall condition $4, I\left(\Phi_{10}\right)$ is finite and positive definite, thus the above difference can be written as

$$
Q\left(\Phi_{1}\right)-Q\left(\Phi_{10}\right)=O_{p}(1) \mu+\frac{1}{2} \mu^{\prime}\left(-I\left(\Phi_{10}\right)+o_{p}(1)\right) \mu+o_{p}(1)
$$

which is dominated by the quadratic term in probability. As a result, for a sufficiently large $C$, there exists a local maximum in the ball $B\left(\Phi_{10}, \frac{1}{\sqrt{m}} u\right)$ with probability $1-\epsilon$, where $\|\mu\|<C$. Alternatively, the result can be expressed as $P\left(\left\|\hat{\Phi}_{1}-\Phi_{10}\right\|<\frac{C}{\sqrt{m}}\right)>1-\epsilon$, and obviously $\hat{\Phi}_{1}$ is a $\sqrt{m}$ consistent estimator of $\Phi_{10}$.

\subsubsection{Convergence Rate}

It has been argued by Fan and $\mathrm{Li}(2003)$ that a procedure is good if it possesses oracle properties. In theorem 1 and 2 , we have showed that the proposed method identifies the true sparse pattern with probability one and coefficients estimator is consistent. In the following theorem, we expect to show that the estimator converges to the true values with a certain rate. 
Theorem 3. For $\lambda_{m} \rightarrow \infty$ and $\frac{\lambda_{m}}{\sqrt{m}} \rightarrow 0, \sqrt{m} I\left(\Phi_{10}\right)\left(\hat{\Phi}_{1}-\Phi_{10}\right) \rightarrow_{d} N\left(0, I\left(\Phi_{10}\right)\right)$, where $I\left(\Phi_{10}\right)$ is the Fisher information knowing that $\Phi_{2}=0$.

Proof. Firstly, Theorem 2 demonstrates that there exists a local maximum for $Q\left(\Phi_{1}\right)$, which is denoted as $\hat{\Phi}_{1}$. It follows that all the partial derivatives for the $\phi_{j} \in \Phi_{1}$ are zero when evaluated at $\hat{\Phi}_{1}$, thus

$$
\left.\frac{\partial}{\partial \Phi_{1}} Q(\Phi)\right|_{\Phi=\left(\hat{\Phi}_{1}, 0\right)^{\prime}}=\left.\frac{\partial}{\partial \Phi_{1}} L(\Phi)\right|_{\Phi=\left(\hat{\Phi}_{1}, 0\right)^{\prime}}-\lambda_{m} h\left(\hat{\Phi}_{1}\right)=0
$$

where $h\left(\hat{\Phi}_{1}\right)=\left(\bar{w}_{1} \operatorname{sgn}\left(\hat{\phi}_{1}\right), \ldots, \bar{w}_{s} \operatorname{sgn}\left(\hat{\phi}_{s}\right)\right)^{\prime}, \bar{w}_{j}=\frac{1}{\hat{\phi}_{j}}$ for $\phi_{j}=\beta_{j}$ or $d_{j}$, and $\bar{w}_{j}=0$ for $\phi_{j}=\gamma_{j}$. Since $h\left(\hat{\Phi}_{1}\right)=h\left(\Phi_{10}\right)$, by using Taylor expansion, we have

$$
\frac{1}{m} \nabla L\left(\Phi_{10}\right)-\left\{I\left(\Phi_{10}\right)+o_{p}(1)\right\}\left(\hat{\Phi}_{1}-\Phi_{10}\right)-\frac{\lambda_{m}}{m} h\left(\Phi_{10}\right)=0
$$

The equation can be transformed as

$$
\sqrt{m}\left\{I\left(\Phi_{10}\right)+o_{p}(1)\right\}\left(\hat{\Phi}_{1}-\Phi_{10}\right)+\frac{\lambda_{m}}{\sqrt{m}} h\left(\Phi_{10}\right)=\frac{1}{\sqrt{m}} \nabla L\left(\Phi_{10}\right) .
$$

Since $E\left(\nabla L\left(\Phi_{10}\right)\right)=0$, the multivariate central limit theorem indicates that

$$
\frac{1}{\sqrt{m}} \nabla L\left(\Phi_{10}\right) \rightarrow_{d} N\left(0, I\left(\Phi_{10}\right)\right)
$$

Consequently,

$$
\sqrt{m} I\left(\Phi_{10}\right)\left(\hat{\Phi}_{1}-\Phi_{10}\right) \rightarrow_{d} N\left(0, I\left(\Phi_{10}\right)\right)
$$

Combining Theorems 1, 2 and 3, we demonstrate that the proposed approach possesses the recommended properties. 


\subsection{Computation Procedure}

Recall that equation (4.8) has the form:

$$
E_{b^{*} \mid \tilde{D}^{(0)}, \tilde{T}^{(0)}}\left\{|| Y-X \beta-\left.Z \tilde{D} \tilde{T} b^{*}\right|^{2}+\lambda_{m}\left(\sum_{j=1}^{p} \frac{\left|\beta_{j}\right|}{\left|\hat{\beta}_{j}\right|}+\sum_{j=1}^{q} \frac{\left|d_{j}\right|}{\left|\hat{d}_{j}\right|}\right)\right\}
$$

and following the earlier discussion, our objective is to write (4.8) as a penalized quadratic term of $(\beta, d)$ and a quadratic term of $\gamma$. Firstly the matrix form of $Z \tilde{D} \tilde{T} b^{*}$ is given by

$$
\begin{aligned}
& Z \tilde{D} \tilde{T} b^{*}=\left(\begin{array}{cccc}
Z_{1} & & & \\
& Z_{2} & & \\
& & \ddots & \\
& & & Z_{m}
\end{array}\right)\left(\begin{array}{cccc}
D & & & \\
& D & & \\
& & \ddots & \\
& & & D
\end{array}\right) \\
& \left(\begin{array}{cccc}
T & & & \\
& T & & \\
& & \ddots & \\
& & & T
\end{array}\right)\left(\begin{array}{c}
b_{1} \\
b_{2} \\
\vdots \\
b_{m}
\end{array}\right),
\end{aligned}
$$

where $\tilde{D}=\operatorname{diag}(D, \ldots, D)$ and $\tilde{T}=\operatorname{diag}(T, \ldots, T)$. More specifically, the matrix $D$ and $T$ are given by

$$
D=\left(\begin{array}{cccc}
d_{1} & & & \\
& d_{2} & & \\
& & \ddots & \\
& & & d_{q}
\end{array}\right), T=\left(\begin{array}{cccc}
1 & & & \\
\gamma_{21} & 1 & & \\
& & \ddots & \\
& & & \\
\gamma_{q 1} & \ldots & \gamma_{q, q-1} & 1
\end{array}\right)
$$

and $b_{i}=\left(b_{i 1}, \ldots, b_{i q}\right)$ for $i=1, \ldots, m$. By the block matrix operation rule, $Z \tilde{D} \tilde{T} b^{*}$ can be 
simplified as

$$
Z \tilde{D} \tilde{T} b^{*}=\left(\begin{array}{c}
Z_{1} D T b_{1} \\
Z_{2} D T b_{2} \\
\vdots \\
Z_{m} D T b_{m}
\end{array}\right)
$$

Note that $D\left(T b_{i}\right)=\operatorname{diag}\left(T b_{i}\right) d$ for $d=\left(d_{1}, \ldots, d_{q}\right)^{\prime}$, then the above matrix can be also written as

$$
\left(\begin{array}{c}
Z_{1} \operatorname{diag}\left(T b_{1}\right) d \\
Z_{2} \operatorname{diag}\left(T b_{2}\right) d \\
\vdots \\
Z_{m} \operatorname{diag}\left(T b_{m}\right) d
\end{array}\right)=\left(\begin{array}{cccc}
Z_{1} & & & \\
& Z_{2} & & \\
& & \ddots & \\
& & & Z_{m}
\end{array}\right) \operatorname{diag}\left(\left(\begin{array}{c}
T b_{1} \\
T b_{2} \\
\vdots \\
T b_{m}
\end{array}\right)\right)\left(\begin{array}{c}
d \\
d \\
\vdots \\
d
\end{array}\right)
$$

where the last vector can be expressed as

$$
\left(\begin{array}{c}
d \\
d \\
\vdots \\
d
\end{array}\right)=\left(\begin{array}{c}
I_{q} \\
I_{q} \\
\vdots \\
I_{q}
\end{array}\right) d=\left(1_{m} \otimes I_{q}\right) d
$$

In the above formula, $1_{m}$ is the column vector with $\mathrm{m} 1$ 's and $\otimes$ is the Kronecker product. In this way, $Z \tilde{D} \tilde{T} b^{*}$ is expressed as a function of $d$, namely $Z \operatorname{diag}\left(\tilde{T} b^{*}\right)\left(1_{m} \otimes I_{q}\right) d$, and the expectation of the whole data takes the form:

$$
E\left(\left\|Y-X \beta-Z \operatorname{diag}\left(\tilde{T} b^{*}\right)\left(1_{m} \otimes I_{q}\right) d\right\|^{2}\right)
$$

In Chapter 3, it has been illustrated that only $\beta$ and $d$ affect the selection result. Therefore, we first treat $\tilde{T}$ as a constant matrix and focus on the minimization of (4.8) with respect to $(\beta, d)$. 


\subsubsection{Update of Fixed and Diagonal Random Effect Estimates}

Regarding equation (4.8), we can simplify the function $\left\|Y-X \beta-Z \operatorname{diag}\left(\tilde{T} b^{*}\right)\left(1_{m} \otimes I_{q}\right) d\right\|^{2}$ as following:

$$
\begin{aligned}
& {\left[Y-X \beta-Z \operatorname{Diag}\left(\tilde{T} b^{*}\right)\left(1_{m} \otimes I_{q}\right) d\right]^{\prime}\left[Y-X \beta-Z \operatorname{Diag}\left(\tilde{T} b^{*}\right)\left(1_{m} \otimes I_{q}\right) d\right] } \\
= & Y^{\prime} Y-2 Y^{\prime}\left[X \beta+Z \operatorname{Diag}\left(\tilde{T} b^{*}\right)\left(1_{m} \otimes I_{q}\right) d\right]+\left[X \beta+Z \operatorname{Diag}\left(\tilde{T} b^{*}\right)\left(1_{m} \otimes I_{q}\right)\right. \\
& d]^{\prime}\left[X \beta+Z \operatorname{Diag}\left(\tilde{T} b^{*}\right)\left(1_{m} \otimes I_{q}\right) d\right]
\end{aligned}
$$

Dropping the constant term regardless of $\beta$ and $d$, the above formula has the matrix form:

$$
\begin{aligned}
& \left(\begin{array}{l}
\beta \\
d
\end{array}\right)^{\prime}\left(\begin{array}{cc}
X^{\prime} X & X^{\prime} Z \operatorname{Diag}\left(\tilde{T} b^{*}\right)\left(1_{m} \otimes I_{q}\right) \\
{\left[X^{\prime} Z \operatorname{Diag}\left(\tilde{T} b^{*}\right)\left(1_{m} \otimes I_{q}\right)\right]^{\prime}} & \bigcirc_{D}
\end{array}\right)\left(\begin{array}{l}
\beta \\
d
\end{array}\right) \\
& -2 Y^{\prime}\left(\begin{array}{ll}
X & Z \operatorname{diag}\left(\tilde{T} b^{*}\right)\left(1_{m} \otimes I_{q}\right)
\end{array}\right)\left(\begin{array}{l}
\beta \\
d
\end{array}\right)
\end{aligned}
$$

where $\bigcirc_{D}=\left(1_{m} \otimes I_{q}\right)^{\prime} \operatorname{Diag}\left(\tilde{T} b^{*}\right) Z^{\prime} Z \operatorname{Diag}\left(\tilde{T} b^{*}\right)\left(1_{m} \otimes I_{q}\right)$. Let $t$ be the iteration index, then the current estimates of $(\tilde{D}, \tilde{T})$ are recorded as $\left(\tilde{D}^{(t)}, \tilde{T}^{(t)}\right)$. Meanwhile, by applying equations (4.5) and (4.6), $E\left(b^{*}\right)$ and $\operatorname{Var}\left(b^{*}\right)$ are both evaluated under the current estimates. Thereafter, by observing the facts that

$$
E\left[X^{\prime} Z \operatorname{Diag}\left(\tilde{T}^{(t)} b^{*}\right)\left(1_{m} \otimes I_{q}\right)\right]=X^{\prime} Z \operatorname{Diag}\left(\tilde{T}^{(t)} E\left(b^{*}\right)\right)\left(1_{m} \otimes I_{q}\right)
$$

and

$$
E\left[Z \operatorname{Diag}\left(\tilde{T}^{(t)} b^{*}\right)\left(1_{m} \otimes I_{q}\right)\right]=Z \operatorname{Diag}\left(\tilde{T}^{(t)} E\left(b^{*}\right)\right)\left(1_{m} \otimes I_{q}\right)
$$

the two expectations above can be obtained by plugging in $E\left(b^{*}\right)$. As a result, the only unknown expectation in (4.22) is $E\left(\bigcirc_{D}\right)$. Regarding $\bigcirc_{D}$, we denote $\circ$ as the element-wise product, then 
the middle part of $\bigcirc_{D}$ can be written as

$$
\operatorname{Diag}\left(\tilde{T} b^{*}\right) Z^{\prime} Z \operatorname{Diag}\left(\tilde{T} b^{*}\right)=\left(Z^{\prime} Z\right) \circ\left(\tilde{T} b^{*}\right)\left(\tilde{T} b^{*}\right)^{\prime}=\left(Z^{\prime} Z\right) \circ\left(\tilde{T} b^{*} b^{* \prime} \tilde{T}^{\prime}\right)
$$

Hence

$$
\bigcirc_{D}=\left(1_{m} \otimes I_{q}\right)^{\prime}\left(Z^{\prime} Z\right) \circ\left(\tilde{T} b^{*} b^{* \prime} \tilde{T}^{\prime}\right)\left(1_{m} \otimes I_{q}\right)
$$

and

$$
E\left(\bigcirc_{D}\right)=\left(1_{m} \otimes I_{q}\right)^{\prime}\left(Z^{\prime} Z\right) \circ\left(\tilde{T} E\left(b^{*} b^{* \prime}\right) \tilde{T}^{\prime}\right)\left(1_{m} \otimes I_{q}\right)
$$

where $E\left(b^{*} b^{* \prime}\right)$ is given by

$$
E\left(b^{*} b^{* \prime}\right)=E\left(b^{*}\right) E\left(b^{* \prime}\right)+\operatorname{Var}\left(b^{*}\right) .
$$

Consequently, $E\left(\bigcirc_{D}\right)$ is also known under $\tilde{T}^{(t)}$, and the expectation of the middle matrix of equation (4.22) is obtained. From the above analysis, we can write equation (4.8) as

$$
\mathrm{L}=\boldsymbol{\eta}^{\prime} \mathbf{M} \boldsymbol{\eta}-2 \boldsymbol{N} \boldsymbol{\eta}+\sum_{i=1}^{p+q} w_{i}\left|\eta_{i}\right|
$$

where $\mathbf{M}$ and $\mathbf{N}$ are given by

$$
\begin{aligned}
& \mathbf{M}=\left(\begin{array}{cc}
X^{\prime} X & X^{\prime} Z \operatorname{Diag}\left(\tilde{T}^{(t)} E\left(b^{*}\right)\right)\left(1_{m} \otimes I_{q}\right) \\
{\left[X^{\prime} Z \operatorname{Diag}\left(\tilde{T}^{(t)} E\left(b^{*}\right)\right)\left(1_{m} \otimes I_{q}\right)\right]^{\prime}} & E\left(\bigcirc_{D}\right)
\end{array}\right), \\
& \mathbf{N}=\left(\begin{array}{ll}
X & \left.Z \operatorname{diag}\left(\tilde{T}^{(t)} E\left(b^{*}\right)\right)\left(1_{m} \otimes I_{q}\right)\right),
\end{array}\right.
\end{aligned}
$$

$\boldsymbol{\eta}^{\prime}$ is defined as $(\beta, d)^{\prime}$, and weights $w_{i}$ 's are given as 


$$
w_{i}= \begin{cases}\frac{\lambda_{m}}{\left|\hat{\beta}_{i}\right|} & \text { if } \eta_{i}=\beta_{i}, \\ \frac{\lambda_{m}}{\left|\hat{d}_{i}\right|} & \text { if } \eta_{i}=d_{i} .\end{cases}
$$

Obviously the form coincides with equation (4.9), then by implementing the algorithm introduced in section 4.2.4, minimization of (4.8) with respect to $(\beta, d)$ is achieved. Denoting the minimizer as $\left(\beta^{(t+1)}, d^{(t+1)}\right)$, then the new objective turns to minimize (4.8) with respect to $\gamma$ with the updated estimates $\left(\beta^{(t+1)}, d^{(t+1)}\right)$.

\subsubsection{Update of Non-diagonal Random Effect Estimates}

For equation (4.8), the part involving $\gamma$ is given by

$$
E\left(Y-X \beta^{(t+1)}-Z \tilde{D}^{(t+1)} \tilde{T} b^{*}\right)^{\prime}\left(Y-X \beta^{(t+1)}-Z \tilde{D}^{(t+1)} \tilde{T} b^{*}\right)
$$

where $\tilde{D}^{(t+1)}$ is generated from $d^{(t+1)}$. Dropping the constant terms regardless of $\gamma,(4.23)$ takes the form:

$$
\begin{aligned}
(4.21)= & E\left(b^{* \prime} \tilde{T}^{\prime} \tilde{D}^{(t+1)^{\prime}} Z^{\prime} Z \tilde{D}^{(t+1)} \tilde{T} b^{*}\right)-2 E\left(b^{* \prime} \tilde{T}^{\prime} \tilde{D}^{(t+1)^{\prime}} Z^{\prime}\left(Y-X \beta^{(t+1)}\right)\right) \\
= & E\left(b^{* \prime}\left(I+\tilde{T}_{0}^{\prime}\right) \tilde{D}^{(t+1)^{\prime}} Z^{\prime} Z \tilde{D}^{(t+1)}\left(I+\tilde{T}_{0}\right) b^{*}\right)-2 E\left(b^{* \prime}\left(I+\tilde{T}_{0}^{\prime}\right) \tilde{D}^{(t+1)^{\prime}} Z^{\prime}\left(Y-X \beta^{(t+1)}\right)\right) \\
= & E\left(b^{* \prime} \tilde{T}_{0}^{\prime} \tilde{D}^{(t+1)^{\prime}} Z^{\prime} Z \tilde{D}^{(t+1)} \tilde{T}_{0} b^{*}\right)+2 E\left(b^{* \prime} \tilde{T}_{0}^{\prime} \tilde{D}^{(t+1)^{\prime}} Z^{\prime} Z \tilde{D}^{(t+1)} b^{*}\right)- \\
& 2 E\left(b^{* \prime} \tilde{T}_{0}^{\prime} \tilde{D}^{(t+1)^{\prime}} \tilde{Z}^{\prime}\left(Y-X \beta^{(t+1)}\right)\right)+\text { Constant, }
\end{aligned}
$$

where $\tilde{T}_{0}=\tilde{T}-I_{m q}$. Recall $\tilde{T}=\operatorname{diag}(T, T \ldots, T)$, then in the same manner, we can write $\tilde{T}_{0}=\operatorname{diag}\left(T_{0}, T_{0} \ldots, T_{0}\right)$. More specifically, $T_{0}$ has the form: 


$$
T_{0}=\left(\begin{array}{cccc}
0 & & & \\
\gamma_{21} & 0 & & \\
& & \ddots & \\
& & & \\
\gamma_{q 1} & \ldots & \gamma_{q, q-1} & 0
\end{array}\right)
$$

Dropping the constant, the three parts of (4.21) are given as following:

$$
\begin{aligned}
& 1=E\left(b^{* \prime} \tilde{T}_{0}^{\prime} \tilde{D}^{(t+1)^{\prime}} Z^{\prime} Z \tilde{D}^{(t+1)} \tilde{T}_{0} b^{*}\right), \\
& 2=2 E\left(b^{* \prime} \tilde{T}_{0}^{\prime} \tilde{D}^{(t+1)^{\prime}} Z^{\prime} Z \tilde{D}^{(t+1)} b^{*}\right), \\
& 3=2 E\left(b^{* \prime} \tilde{T}_{0}^{\prime} \tilde{D}^{(t+1)^{\prime}} \tilde{Z}^{\prime}\left(Y-X \beta^{(t+1)}\right)\right) .
\end{aligned}
$$

Regarding equation (4.24), note that

$$
\tilde{T}_{0} b^{*}=\left(\begin{array}{c}
T_{0} b_{1}^{*} \\
T_{0} b_{2}^{*} \\
\vdots \\
T_{0} b_{m}^{*}
\end{array}\right)
$$

and for all $T_{0} b_{i}{ }^{*}(i=1,2, \ldots, m)$, they can be transformed as

$$
T_{0} b_{i}^{*}=\mathrm{B}_{i} \gamma
$$

In the above formula, $\gamma=\left(\gamma_{21}, \gamma_{31}, \gamma_{32}, \ldots, \gamma_{q, q-1}\right)^{\prime}$, and $\mathrm{B}_{i}$ is given by 


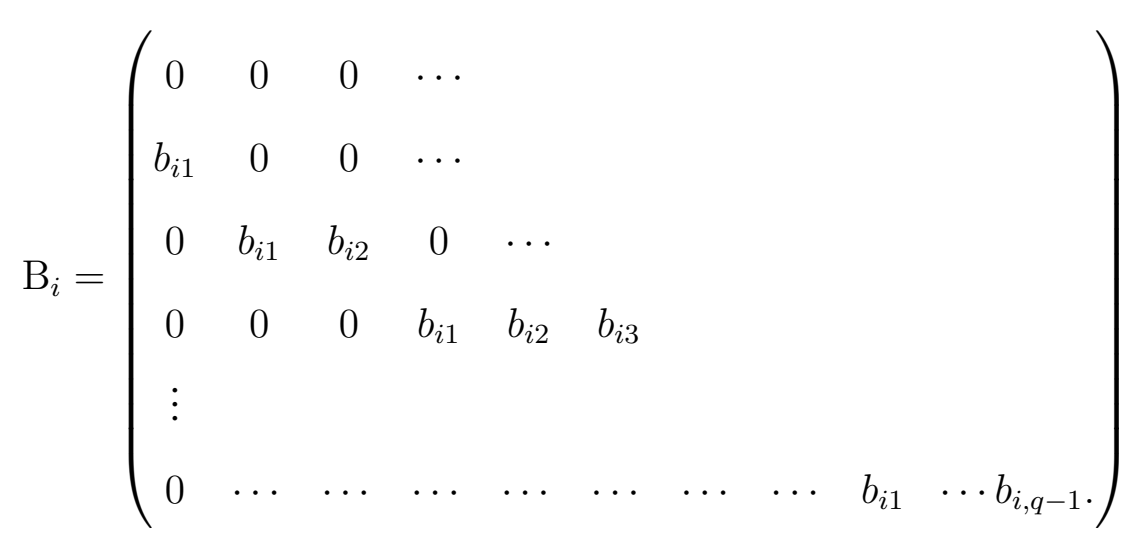

Consequently, $b_{i}^{* \prime} T_{0}^{\prime} D^{(t+1)^{\prime}} Z_{i}^{\prime} Z_{i} D^{(t+1)} T_{0} b_{i}^{*}=\gamma^{\prime} \mathrm{B}_{i}^{\prime} D^{(t+1)^{\prime}} Z_{i}^{\prime} Z_{i} D^{(t+1)} \mathrm{B}_{i} \gamma$, and equation (4.24) is written as

$$
\text { (1) }=\sum_{i=1}^{m} E\left(\gamma^{\prime} \mathrm{B}_{i}^{\prime} D^{(t+1)^{\prime}} Z_{i}^{\prime} Z_{i} D^{(t+1)} \mathrm{B}_{i} \gamma\right) \text {. }
$$

Let $b_{i}=\left(b_{i 1}{ }^{*}, b_{i 1}{ }^{*}, b_{i 2}{ }^{*}, \ldots, b_{i 1}{ }^{*}, b_{i 2}{ }^{*}, \ldots, b_{i, q-1}{ }^{*}\right)^{\prime}, d=\left(d_{2}^{(t+1)}, d_{3}^{(t+1)}, d_{3}^{(t+1)}, \ldots, d_{q}^{(t+1)}, \ldots, d_{q}^{(t+1)}\right)^{\prime}$ and

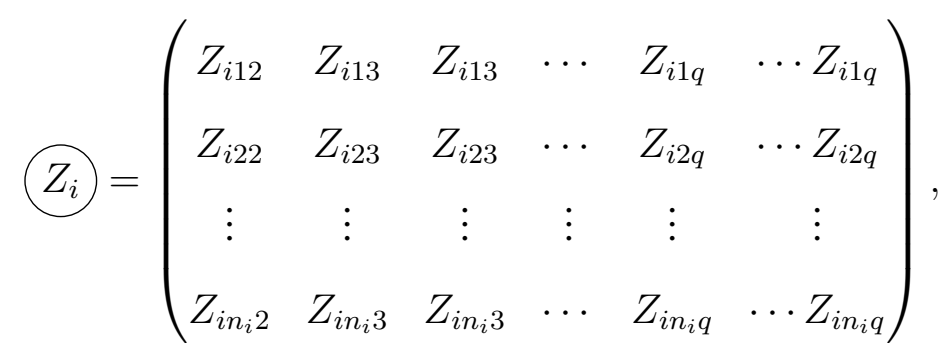

it follows $\left.\left.\mathrm{B}_{i}^{\prime} D^{(t+1)^{\prime}} Z_{i}^{\prime} Z_{i} D^{(t+1)} \mathrm{B}_{i}=\left(b_{i} \bullet b_{i}\right)^{\prime} \circ(d) \bullet(d)^{\prime}\right) \circ\left(Z_{i}\right)^{\prime} \bullet Z_{i}\right)$, where $\circ$ has been earlier defined as the element-wise product. To distinguish it from inner product, we specify inner product as $\bullet$. Therefore, 1 is given by

$$
\text { (1) } \left.\left.=\sum_{i=1}^{m} \gamma^{\prime} E\left(\left(b_{i} \cdot b_{i}\right)^{\prime}\right) \circ\left(d \cdot(d)^{\prime}\right) \circ\left(Z_{i}\right)^{\prime} \cdot Z_{i}\right)\right) \gamma \text {, }
$$

where $\left.\left.E\left(b_{i} \cdot b_{i}\right)^{\prime}\right)=E\left(b_{i}\right) \bullet E\left(b_{i}\right)^{\prime}\right)+\operatorname{Var}\left(b_{i}\right)$. Since $E\left(b_{i}\right)$ and $\operatorname{Var}\left(b_{i}\right)$ can be derived from $E\left(b_{i}\right)$ and $\operatorname{Var}\left(b_{i}\right)$, (1) turns to be a known quadratic term of $\gamma$. Specifically, its matrix form 
is given as

$$
\text { (1) }=\gamma^{\prime} \bullet \mathrm{I}^{\prime} \bullet E\left(\left(\mathrm{~b} \cdot \mathrm{b}^{\prime}\right) \circ\left(\tilde{d} \cdot(\tilde{d})^{\prime}\right) \circ\left(\mathrm{Z} \cdot \mathrm{Z}^{\prime}\right)\right) \bullet \mathrm{I} \cdot \gamma
$$

where $\mathrm{b}=\operatorname{diag}\left(b_{1}, b_{2}, \ldots, b_{m}\right), \tilde{d}=\operatorname{diag}(\underbrace{(\mathrm{d}),(\mathrm{d}), \ldots, \mathrm{d})}_{\mathrm{m}}, \mathbf{Z}=\operatorname{diag}\left(Z_{1}, Z_{2}\right.$, $\left.\ldots, Z_{m}\right)$ and $\mathrm{I}=1_{m} \otimes I_{q(q-1) / 2}$.

Next, analyzing equation (4.25), we have the following results:

$$
\begin{aligned}
& \text { (2) }=2 E\left(b^{* \prime} \tilde{T}_{0}^{\prime} \tilde{D}^{(t+1)^{\prime}} Z^{\prime} Z \tilde{D}^{(t+1)} b^{*}\right)
\end{aligned}
$$

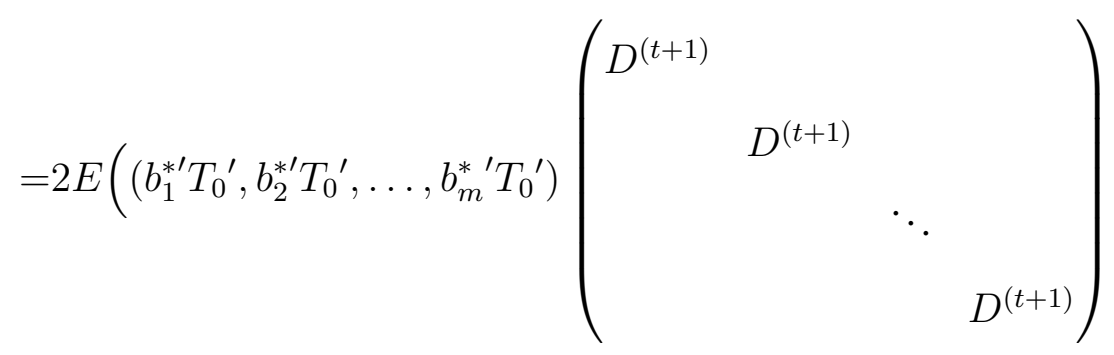

$$
\begin{aligned}
& \left(\begin{array}{cccc}
Z_{1}^{\prime} Z_{1} & & & \\
& Z_{2}^{\prime} Z_{2} & & \\
& & \ddots & \\
& & & Z_{m}{ }^{\prime} Z_{m}
\end{array}\right)\left(\begin{array}{ccc}
D^{(t+1)} & & \\
& D^{(t+1)} & \\
& \ddots & \\
& & D^{(t+1)}
\end{array}\right)\left(\begin{array}{c}
b_{1}{ }^{*} \\
b_{2}{ }^{*} \\
\vdots \\
b_{m}{ }^{*}
\end{array}\right) \\
& =2 E\left(\sum_{i=1}^{m} b_{i}^{* \prime} T_{0}{ }^{\prime} D^{(t+1)} Z_{1}{ }^{\prime} Z_{1} D^{(t+1)} b_{i}{ }^{*}\right) \\
& =2 E\left(\sum_{i=1}^{m} \gamma^{\prime} B_{i}{ }^{(t+1)} Z_{1}{ }^{\prime} Z_{1} D^{(t+1)} b_{i}^{*}\right) \\
& \left.=2 E\left(\sum_{i=1}^{m} \gamma^{\prime} \bullet\left[\left(Z_{i}\right)^{\prime} \bullet Z_{i}\right) \circ\left(b_{i} \bullet b_{i}{ }^{\prime}\right) \circ\left(\mathrm{d} \bullet 1_{q}{ }^{\prime}\right)\right] \bullet d\right) \text {. }
\end{aligned}
$$

In particular, $1_{q}=(\underbrace{1,1, \ldots, 1}_{\mathrm{q}})^{\prime}$ in the above formula, and its matrix expression is given by

$$
\text { (2) }=2 \gamma^{\prime} \bullet \mathrm{I}^{\prime} \bullet\left[\left(\mathrm{Z}^{\prime} \bullet Z\right) \circ E\left(\mathrm{~b} \cdot\left(\tilde{b}^{\prime}\right) \circ\left(\tilde{d} \cdot 1_{q}^{\prime}\right)\right] \bullet \mathrm{I}_{0} \bullet d\right.
$$


where $\left(\tilde{b}^{\prime}=\operatorname{diag}\left(b_{1}{ }^{\prime}, b_{2}{ }^{\prime}, \ldots . b_{m}{ }^{\prime}\right), \hat{1}_{q}{ }^{\prime}=\operatorname{diag}(\underbrace{1_{q^{\prime}}, 1_{q}{ }^{\prime}, \ldots, 1_{q}{ }^{\prime}}_{\mathrm{m}})\right.$ and $\mathrm{I}_{0}=1_{m} \otimes I_{q}$.

Finally focusing on equation (4.26), it can be transformed as

$$
\begin{aligned}
& \text { (3) }=2 E\left(b^{* \prime} \tilde{T}_{0}^{\prime} \tilde{D}^{(t+1)^{\prime}} \tilde{Z}^{\prime}\left(Y-X \beta^{(t+1)}\right)\right) \\
& =2 E\left(\left(b_{1}^{* \prime} T_{0}{ }^{\prime}, b_{2}^{* \prime} T_{0}{ }^{\prime}, \ldots, b_{m}^{* \prime} T_{0}{ }^{\prime}\right)\left(\begin{array}{cccc}
D^{(t+1)} & & & \\
& D^{(t+1)} & & \\
& & \ddots & \\
& & & D^{(t+1)}
\end{array}\right)\left(\begin{array}{llll}
Z_{1}{ }^{\prime} & & & \\
& Z_{2}{ }^{\prime} & & \\
& & \ddots & \\
& & & Z_{m}{ }^{\prime}
\end{array}\right)\right. \\
& \text { - } \left.\left(Y-X \beta^{(t+1)}\right)\right) \\
& =2 E\left(\left(b_{1}^{* \prime} T_{0}{ }^{\prime} D Z_{1}{ }^{\prime}, b_{2}^{* \prime} T_{0}{ }^{\prime} D Z_{2}{ }^{\prime}, \ldots, b_{m}^{* \prime} T_{0}{ }^{\prime} D Z_{m}{ }^{\prime}\right)\left(Y-X \beta^{(t+1)}\right)\right) \\
& =2 E\left(\left(\gamma^{\prime} B_{1}{ }^{\prime} D Z_{1}{ }^{\prime}, \gamma^{\prime} B_{2}{ }^{\prime} D Z_{2}{ }^{\prime}, \ldots, \gamma^{\prime} B_{m}{ }^{\prime} D Z_{m}{ }^{\prime}\right)\left(Y-X \beta^{(t+1)}\right)\right) \\
& \left.=2 E(\underbrace{\left(\gamma^{\prime}, \gamma^{\prime}, \ldots, \gamma^{\prime}\right.}_{\mathrm{m}})\left(\begin{array}{llll}
B_{1}{ }^{\prime} D^{(t+1)} Z_{1}{ }^{\prime} & & & \\
& B_{2}{ }^{\prime} D^{(t+1)} Z_{2}{ }^{\prime} & & \\
& & \ddots & \\
& & & B_{m}{ }^{\prime} D^{(t+1)} Z_{m}{ }^{\prime}
\end{array}\right)\left(Y-X \beta^{(t+1)}\right)\right) \text {. }
\end{aligned}
$$

Therefore, the stacked matrix expression is

$$
\text { (3) }=2 \gamma^{\prime} \cdot(\mathrm{I})^{\prime} \bullet E\left(\mathrm{~B}^{\prime}\right) \cdot \tilde{D}^{(t+1)} \bullet Z^{\prime} \bullet\left(Y-X \beta^{(t+1)}\right) \text {, }
$$

where ${ }^{\prime}{ }^{\prime}=\operatorname{diag}\left(B_{1}{ }^{\prime}, B_{2}{ }^{\prime}, \ldots, B_{m}{ }^{\prime}\right)$. Define matrices $P, G$ and $H$ as following:

$$
\begin{aligned}
& P=(\mathrm{I})^{\prime} \cdot E\left(\left(\mathrm{~b} \cdot\left(\mathrm{b}^{\prime}\right) \circ\left(\tilde{d} \cdot(\tilde{d})^{\prime}\right) \circ\left(\mathrm{Z} \cdot\left(\mathrm{Z}^{\prime}\right)\right) \cdot \mathrm{I},\right.\right. \\
& G=\mathrm{I}^{\prime} \bullet\left[\left(\left(\mathbf{Z}^{\prime} \bullet Z\right) \circ E\left(\left(\mathrm{~b} \cdot \tilde{b^{\prime}}\right) \circ\left(\tilde{d} \cdot\left(1_{q}^{\prime}\right)\right)\right] \bullet \mathrm{I}_{0} \bullet d,\right.\right. \\
& H=\mathrm{I}^{\prime} \bullet E\left(\mathrm{~B}^{\prime}\right) \bullet \tilde{D}^{(t+1)} \bullet Z^{\prime} \bullet\left(Y-X \beta^{(t+1)}\right),
\end{aligned}
$$


then (4.23) is given as

$$
g(\gamma)=\gamma^{\prime} P \gamma+2 \gamma^{\prime}(G-H)+\text { constant }
$$

Since $E\left(\mathrm{~b} \cdot \mathrm{b}^{\prime}\right), E\left(\mathrm{~b} \cdot \tilde{b}^{\prime}\right)$ and $\left.\mathrm{E}(\mathrm{B})^{\prime}\right)$ are all known matrices, $P, G, H$ can be also obtained under current estimates. To minimize $g(\gamma)$, setting the derivative as 0 , we have the equation:

$$
\frac{\partial g}{\partial \gamma}=2 P \gamma+2(G-H)=0
$$

Since $P$ is positive definite, its inverse always exists, and the solution for $\gamma$ takes the form: $P^{-1}(H-G)$, which is recored as $\gamma^{(t+1)}$.

Combining sections 4.4.1 and 4.4.2, a whole iteration is completed and we obtain the new estimates $\left(\beta^{(t+1)}, d^{(t+1)}, \gamma^{(t+1)}\right)$. Afterwards, repeat algorithms in sections 4.4.1 and 4.4.2 until the estimates converge, and record the final results, namely $(\hat{\beta}, \hat{d}, \hat{\gamma})$. In this way, the global minimum of (4.8) with a preassigned $\lambda_{m}$ can be attained. Because of the shrinkage property, some estimates are zero, and we may obtain the simplified model.

\subsubsection{Fixed Effects Screening}

In the last several sections, we have demonstrated the effectiveness of the proposed approach. Nevertheless, empirical results indicate that some small coefficients still frequently appear in the final model, which causes the over-selection problem. In particular, this issue is more distinct in the selection of fixed effects. By observing the simulation results, it shows that the corrected selection rate of fixed effects is always relatively lower compared to the corrected selection rate of random effects.

The over-selection may come from three perspectives. Firstly, although the method possesses continuously shrinkage property, $\lambda$ is incapable of being shifted continuously in the preassigned interval. Instead, $\lambda$ are assigned with discrete values, and the best " $\lambda$ " may happen to be between two values. As a result, some information is lost when the method is employed. Additionally, because of computing limitation, the threshold is set to be $10^{-3}$ to determine the convergence of 
$\left(\beta^{(t)}, d^{(t)}, \gamma^{(t)}\right)$. Therefore, the computation inaccuracy may cause the bias of estimating $(\hat{\beta}, \hat{d}, \hat{\gamma})$. Finally, theoretical results only ensure the consistency in asymptotic case, while the sample size is always finite in practice. Consequently, the selection result may differ from our expectation.

To eliminate noisy factors of fixed effects, a screening step is recommended for the candidate model. Some popular tests are listed as follows.

1. Wald test: a test of the effect size which is scaled using the estimated standard error.

2. LRT (Likelihood ratio test): it tests the difference in two nested models using the chi-square distribution.

3. Parametric bootstrap test: the test assumes the model which restricts a parameter to zero (null model) is the true distribution and generates an empirical distribution of the difference in the two models. The observed coefficient is tested against the generated empirical distribution.

With the assumptions of asymptotic distributions and independent predictors, Wald and LRT tests are equivalent. However, when a sample size is not large enough to be a good approximation of the asymptotic distribution or there is some correlation among the predictors, Wald and LRT test results can vary considerably. In practice, LRT test is generally preferred over Wald test for fixed effects in mixed models. Regarding parametric bootstrap, it is considered to be the most reliable test for fixed effects in mixed models. However, the test is more computationally expensive and requires longer run times. To compromise between reliance and efficiency, we choose the LRT test to screen the fixed effects.

Specifically, we first employ adaptive lasso via cross-validation to obtain the candidate model with smallest mBIC. For the candidate model, drop one fixed effect at one time. Let the maximum likelihood of full model be $L_{f u l l}$, and the maximum likelihood of the reduced model be $L_{-i}$, where $i$ indicates dropping the $i$ th fixed effect. To obtain a test statistic, we compute the maximum likelihood ratio between reduced model and full model, and denote the ratio as $R_{i}$, where a smaller 
$R_{i}$ means a smaller difference between two models. Thus $R_{i}$ is given by

$$
R_{i}=\frac{L_{-i}}{L_{\text {full }}}
$$

Afterwards, transforming the maximum likelihood ratio to obtained the chi-square statistic $\chi_{i}^{2}$, it is given by

$$
\chi_{i}^{2}=-2 \ln R_{i}
$$

When the difference between two nested models is significant, $R_{i}$ would be relatively small and $\chi_{i}^{2}$ would be relatively large. In addition, the degree of freedom lost each time is 1 , therefore the p-value can be computed by

$$
\mathrm{p}-\text { value }=\operatorname{Pr}\left(\chi_{d f=1}^{2}>\chi_{i}^{2}\right)
$$

Setting the cutoff at 0.05 , we adopt all the fixed effects with p-values less than 0.05 as significant effects, while those effects with p-values greater than 0.05 are dropped. In this way, some noisy factors are eliminated from the candidate model.

Furthermore, empirical results indicate that the power of identifying true non-zero effects almost stay the same when the noisy factors are dropped. In the other words, true significant effects are rarely eliminated by screening step. Consequently, it is convincing that the screening step effectively reduces over-selection rate while keeping a relatively high power.

Generally speaking, either theoretical properties or empirical analysis are addressed to confirm the good performance of adaptive lasso via cross-validation, and fixed effects are screened at the end to even reduce the over selection rate. In next chapter, we will see several simulation results to illustrate it more explicitly. 


\section{CHAPTER 5 SIMULATON STUDY}

In this simulation study, we implement the adaptive lasso via cross-validation approach under different settings to detect the model selection accuracy. The proposed approach is good if it possesses the following properties:

1. Generality: the proposed approach is available under general settings, regardless the underlying distribution of the design matrix.

2. Consistency: it has been proved that the selection result is consistent, hence the correct selection rate tends to increase as the sample size increases.

3. Feasibility: since practically the sample size is always finite, we expect that the proposed approach keeps a relatively high rate even for a small sample.

To justify these properties, different distributions are considered to generate the design matrix. Meanwhile, the sample size is increased from moderate small to relatively large. Furthermore, by comparing the selection results with some existing approaches, we demonstrate that the adaptive lasso via cross-validation outperforms other methods, especially in small samples.

\subsection{Presentation of Simulations}

Recall linear mixed model (3.1) is given by

$$
\boldsymbol{y}_{\boldsymbol{i}}=\mathbf{X}_{\mathrm{i}} \boldsymbol{\beta}+\mathbf{Z}_{\mathbf{i}} b_{i}+\epsilon_{i},
$$

for the $i$ th subject, where $\mathbf{X}_{\mathbf{i}}$ and $\mathbf{Z}_{\mathbf{i}}$ are design matrices of fixed and random effects respectively, and $\epsilon_{i}$ is error term. To test generality, we first generate both of $\mathbf{X}_{\mathbf{i}}$ and $\mathbf{Z}_{\mathbf{i}}$ from a uniform distribution. Afterwards, both matrices are generated from a normal distribution. Regarding the sample size, let $m$ denote the sample size. We take $m=30,60$ and 90 to see how the performance changes as sample size increases. Additionally, considering the number in each subgroup may also affect 
the selection accuracy, we adopt different subgroup size in the simulation. For convenience, only balanced data are taken into account. Therefore, the subgroup size regarding each subject is identical. Recall the notations in model (3.1), where $n_{i}$ represents the subgroup size of the $i$ th subject, then $n_{1}=n_{2}=\ldots=n_{m}$. Let $n$ be the subgroup size of each subject, and set $n$ to either 5 or 10 . Regarding the number of effects, recall $p$ represents the number of fixed effects and $q$ represents the number of random effects.

To evaluate the efficiency, we present several indices used later. The notations and their meanings are summarized in the following table:

\begin{tabular}{|l|l|}
\hline Abbreviation & Full Name \\
\hline C & Correct selection rate of the true model \\
\hline CF & Correct selection rate of the fixed effect \\
\hline CR & Correct selection rate of the random effect \\
\hline FP & False positive rate \\
\hline OVER & The rate of selecting exact or overfitting models \\
\hline
\end{tabular}

To keep uniformly in simulation studies, we conduct 130 replications of each model, and summarize their results in tables.

1. First of all, we consider the case that $p=q=4$. Let $\beta=(0,0,0,0)$ and random effects $\boldsymbol{b}_{\boldsymbol{i}}$ take the covariance

$$
\left(\begin{array}{llll}
4 & 0 & 0 & 0 \\
0 & 4 & 0 & 0 \\
0 & 0 & 4 & 0 \\
0 & 0 & 0 & 0
\end{array}\right) .
$$


Following the notations in (3.1), $y_{i j}$ is generated by

$$
y_{i j}=b_{i 1}+z_{i j 2} b_{i 2}+z_{i j 3} b_{i 3}+z_{i j 4} b_{i 4}+\epsilon_{i j}
$$

where the error terms $\epsilon_{i j}{ }^{\prime} s$ are independently drawn from the standard normal distribution $\mathrm{N}(0,1)$. For the random design matrix in equation (5.1), random intercept is included, therefore $z_{i j 1}^{\prime} s$ are all $1^{\prime} s$. Regarding the other columns of $\mathbf{Z}_{\mathbf{i}}$, namely $z_{i j 2}^{\prime} s, z_{i j 3}^{\prime} s$ and $z_{i j 4}^{\prime} s$, they are independently drawn from the uniform distribution $U(-1,1)$. Let CV.ALASSO represent the adaptive lasso via cross-validation, simulation results are given in the following Table 5.1.

Table 5.1: Uniform design matrix with $n=5$

\begin{tabular}{llllll}
\hline $\mathrm{m}$ & Method & $\mathrm{C}$ & $\mathrm{CF}$ & $\mathrm{CR}$ & $\mathrm{FP}$ \\
\hline 30 & CV.ALASSO & $78.84 \%$ & $82.06 \%$ & $90.19 \%$ & $4.93 \%$ \\
\hline 60 & CV.ALASSO & $84.53 \%$ & $87.44 \%$ & $93.47 \%$ & $4.60 \%$ \\
\hline 90 & CV.ALASSO & $86.30 \%$ & $88.97 \%$ & $93.81 \%$ & $4.44 \%$ \\
\hline
\end{tabular}

In general, the results indicate that selection accuracy is improved as sample size increases. The corrected selection rates of the true model, fixed and random effects are all higher for a larger sample, while the false positive rate decreases. However, we also observe that the improvement from 30 to 60 is less distinct than the improvement from 60 to 90, which shows the improvement trend decreases for a larger sample.

2. Secondly, we implement the proposed approach under the settings of Bondell (2010), where $p=9$ and $q=4$. Let $\beta_{1}=\beta_{2}=1, \beta_{3}=\beta_{4}=\ldots=\beta_{9}=0$, and the covariacne structure of the random effects is 


$$
V^{(2)}=\left(\begin{array}{cccc}
9 & 4.8 & 0.6 & 0 \\
4.8 & 4 & 1 & 0 \\
0.6 & 1 & 1 & 0 \\
0 & 0 & 0 & 0
\end{array}\right)
$$

In this model, random effects are correlated, and the generating model takes the form:

$$
y_{i j}=x_{i j 1} \beta_{1}+x_{i j 2} \beta_{2}+\ldots+x_{i j 9} \beta_{9}+b_{i 1}+z_{i j 2} b_{i 2}+z_{i j 3} b_{i 3}+z_{i j 4} b_{i 4}+\epsilon_{i j} .
$$

Similar to (5.1), $\mathbf{X}_{\mathbf{i}}$ and $\mathbf{Z}_{\mathbf{i}}$ are generated from uniform distribution $U(-1,1)$, and $\epsilon_{i j}^{\prime} s$ are independently and identically drawn from $N(0,1)$. For random effects $b_{i 4}{ }^{\prime} s$, they are unimportant random effects corresponding to 0 . By employing adaptive lasso via cross-validation with 150 repetitions, we obtain the results in Table 5.2. Moreover, the approach proposed in Bondell's paper without cross-validation or screening is also presented in the table to make a comparison.

Table 5.2: Uniform design matrix with $n=5$

\begin{tabular}{lllll}
\hline $\mathrm{m}$ & Method & $\mathrm{C}$ & $\mathrm{CF}$ & $\mathrm{CR}$ \\
\hline 30 & CV.ALASSO & $75.86 \%$ & $79.31 \%$ & $89.37 \%$ \\
\hline 30 & ALASSO.BIC & $71 \%$ & $73 \%$ & $79 \%$ \\
\hline
\end{tabular}

By comparing the results of two approaches, we can tell that CV.ALASSO outperforms ALASSO.BIC, and all the corrected selection rates increase. Meanwhile, comparing the first row of Table 5.2 to the first row of Table 5.1, all the corrected selection rates are roughly the same. According to this result, it is plausible to believe that CV.ALASSO is relatively insensitive to the underlying model.

To detect sample size's effect of CV.ALASSO in model (5.2), we increase $m$ to 60 and 90, and the results are given in Table 5.3. 
Table 5.3: Uniform design matrix with $n=5$

\begin{tabular}{lllllll}
\hline $\mathrm{m}$ & Method & $\mathrm{C}$ & $\mathrm{CF}$ & $\mathrm{CR}$ & $\mathrm{FP}$ & OVER \\
\hline 30 & CV.ALASSO & $75.86 \%$ & $79.31 \%$ & $89.37 \%$ & $5.24 \%$ & $91.53 \%$ \\
\hline 60 & CV.ALASSO & $83.72 \%$ & $85.05 \%$ & $95.02 \%$ & $4.76 \%$ & $97.69 \%$ \\
\hline 90 & CV.ALASSO & $86.37 \%$ & $90.12 \%$ & $96.92 \%$ & $4.58 \%$ & $96.92 \%$ \\
\hline
\end{tabular}

Table 5.3 shows a similar trend as Table 5.1, where all the corrected selection rates increase as sample size increases. Particularly, the improvement from 60 to 30 is more distinct than the improvement from 60 to 90 .

3. Thirdly, we modify the generating model in (5.2), where fixed effects are $\beta=(1,1,0, \ldots, 0)$ and covariance of random effects is $V^{(2)}$. However, now let $x_{i j}{ }^{\prime} s$ be independently drawn from the normal distribution $\mathbf{N}(0,1)$, while the elements of $\mathbf{Z}$ are still drawn from the uniform distribution $U(-1,1)$. We call this modified generating model as model (5.3), and its simulation results are provided as follows.

Table 5.4: Normal design matrix with $n=5$

\begin{tabular}{lllllll}
\hline $\mathrm{m}$ & Method & $\mathrm{C}$ & $\mathrm{CF}$ & $\mathrm{CR}$ & $\mathrm{FP}$ & OVER \\
\hline 30 & CV.ALASSO & $74.04 \%$ & $77.12 \%$ & $92.36 \%$ & $5.87 \%$ & $97.69 \%$ \\
\hline 60 & CV.ALASSO & $80.37 \%$ & $83.59 \%$ & $92.33 \%$ & $5.06 \%$ & $98.46 \%$ \\
\hline 90 & CV.ALASSO & $82.29 \%$ & $87.83 \%$ & $97.67 \%$ & $4.98 \%$ & $98.46 \%$ \\
\hline
\end{tabular}

Comparing Table 5.4 and Table 5.3, it indicates that CV.ALASSO keeps similar corrected selection rates for both models, which again demonstrates the insensitiveness of the proposed approach with respect to design matrix. 
4. Afterwards, we investigate the effects of subgroup size. Increasing the subgroup size $n$ from 5 to 10 and employing the same generating model in (5.2), simulation results are presented in Table 5.5.

Table 5.5: Uniform design matrix with $n=10$

\begin{tabular}{lllllll}
\hline $\mathrm{m}$ & Method & $\mathrm{C}$ & $\mathrm{CF}$ & $\mathrm{CR}$ & $\mathrm{FP}$ & OVER \\
\hline 30 & CV.ALASSO & $81.37 \%$ & $82.90 \%$ & $95.29 \%$ & $5.10 \%$ & $96.15 \%$ \\
\hline 60 & CV.ALASSO & $87.02 \%$ & $89.65 \%$ & $95.40 \%$ & $5.23 \%$ & $100 \%$ \\
\hline 90 & CV.ALASSO & $89.53 \%$ & $90.27 \%$ & $96.92 \%$ & $4.17 \%$ & $96.92 \%$ \\
\hline
\end{tabular}

Firstly, we observe the fact in accordance with Table 5.3, where all the corrected selection rates increase as the sample size $m$ increases, and improvement trend becomes less distinct from 60 to 90. On the other hand, all the corrected selection rates in Table (5.5) are relatively higher than those in Table (5.3), from which we can conclude that the increment of subgroup size improves selection accuracy.

Furthermore, comparing CV.ALASSO to the approach without cross-validation for model (5.2), when $n=10$ and $m=60$, we summarize results in Table 5.6.

Table 5.6: Uniform design matrix with $n=10$

\begin{tabular}{lllll}
\hline $\mathrm{m}$ & Method & $\mathrm{C}$ & $\mathrm{CF}$ & $\mathrm{CR}$ \\
\hline 60 & CV.ALASSO & $87.02 \%$ & $89.65 \%$ & $95.40 \%$ \\
\hline 60 & ALASSO.BIC & $83 \%$ & $83 \%$ & $83 \%$ \\
\hline
\end{tabular}

Table 5.6 demonstrates that all the corrected selection rates increase, therefore CV.ALASSO outperforms the existing approach, ALASSO.BIC, in this setting. 
5. Next, consider the relatively high dimensional case. Let $p=9$ and $q=10$, where $\boldsymbol{\beta}=$ $(1,1,0, \ldots, 0)$ and covariance matrix is 10 by 10 with the form:

$$
V^{(5)}=\left(\begin{array}{cccccc}
9 & 4.8 & 0.6 & 0 & \ldots & 0 \\
4.8 & 4 & 1 & 0 & \ldots & 0 \\
0.6 & 1 & 1 & 0 & \ldots & 0 \\
\vdots & \vdots & \vdots & \vdots & \vdots & \vdots \\
0 & 0 & 0 & 0 & \ldots & 0
\end{array}\right)
$$

Taking uniform design matrices for both fixed and random effects, the generating model is given by

$$
y_{i j}=x_{i j 1} \beta_{1}+x_{i j 2} \beta_{2}+\ldots+x_{i j 9} \beta_{9}+b_{i 1}+z_{i j 2} b_{i 2}+z_{i j 3} b_{i 3}+\ldots+z_{i j 10} b_{i 10}+\epsilon_{i j} .
$$

We denote this model as model (5.4), and the error terms $\epsilon_{i j}{ }^{\prime} s$ in this model are still drawn from $\mathrm{N}(0,1)$. When sample size $m=60$ and subgroup size $n=30$, simulation results of CV.ALASSO and ALASSO.BIC are presented as follows.

Table 5.7: Uniform design matrix with $m=60, n=10, q=10$

\begin{tabular}{llll}
\hline & $\mathrm{C}$ & $\mathrm{CF}$ & $\mathrm{CR}$ \\
\hline ALASSO.BIC & $61 \%$ & $63 \%$ & $84 \%$ \\
\hline CV.ALASSO & $60.57 \%$ & $62.08 \%$ & $87.46 \%$ \\
\hline
\end{tabular}

Comparing to Table 5.6, all the corrected selection rates significantly decrease. Particularly, the reduction rates for true model and fixed effects are even greater than $20 \%$. Therefore, both approaches perform poorer when the dimension of random effects is relatively high. Next, comparing the performance of two approaches in this setting, Table 5.7 shows that there is no distinct differ- 
ence. Consequently, neither approach performs very well when the dimension of random effects is relatively high.

6. To further discuss the generality of our approach, we replace the $\beta$ in model (5.2) with triangular vector: $\beta=c(3: 1, \operatorname{rep}(0,6))$, and maintain the same covariance matrix $V^{(2)}$. Regarding the design matrix, we still utilize uniform design matrix for both fixed and random effects. Setting subgroup size $n=10$ and sample size $m=30,60,90$, simulation results are presented in Table 5.8 .

Table 5.8: Uniform design matrix with $n=10$

\begin{tabular}{llllll}
\hline $\mathrm{m}$ & Method & $\mathrm{C}$ & $\mathrm{CF}$ & $\mathrm{CR}$ & $\mathrm{FP}$ \\
\hline 30 & CV.ALASSO & $75.65 \%$ & $77.59 \%$ & $93.29 \%$ & $6.18 \%$ \\
\hline 60 & CV.ALASSO & $79.33 \%$ & $82.08 \%$ & $95.40 \%$ & $5.47 \%$ \\
\hline 90 & CV.ALASSO & $81.06 \%$ & $84.19 \%$ & $94.49 \%$ & $5.33 \%$ \\
\hline
\end{tabular}

Comparing to Table 5.5, simulation results indicate that corrected selection rates for the true model and fixed effects are slightly lower, while corrected rates for random effects are almost the same. Regarding the sample size's effects, we can see that all the corrected selection rates are relatively higher, and false positive rates are lower when sample size increases. Overall, it is still acceptable to conclude that CV.ALASSO possesses generality regardless of fixed effects in the underlying model.

\subsection{Conclusion}

In this simulation study, we demonstrate that the proposed approach efficiently identifies the sparse pattern of the true model in most cases, and selects fixed effects and random effects simultaneously with a high rate.

Furthermore, when compared to adaptive lasso without cross-validation, our approach often 
possesses a higher corrected selection rate. More specifically, it reduces the over-selection rate for fixed effects while keeping a high probability to identify all the significant factors, which improves the efficiency of the existing approach.

In addition, among all different underlying models, either increment of sample size or subgroup size improves the selection accuracy, which agrees with consistency property. Therefore, a larger sample or subgroup size is always preferred practically.

By comparing the results under different models, with different design matrices, fixed effects, or even covariance of random effects, corrected selection rates are roughly the same. As a result, the procedure is generally stable regardless of the underlying model.

Though the approach generally possesses the recommended properties listed at the beginning of this chapter, there are still some drawbacks. For instance, the method does not perform very well in a high dimensional case, and one possible reason is that the estimated covariance is inaccurate when the number of random effects is relatively large. To develop a procedure more efficient in high dimensional case, we may need some further discussions. 


\section{CHAPTER 6 APPLICATION}

In chapter 4 and 5 , it has been argued that adaptive lasso via cross-validation achieves variable selection in linear mixed models with a relatively high rate. In this chapter, we will mainly discuss the application of this approach in a very classic real data example, Growth curve on an orthodontic measurement.

\subsection{Data Description}

Growth curve data is collected form 27 children from age 8 until age 14, and among them, there are 16 males and 11 females. The distance (in $\mathrm{mm}$ ) between the pituitary and the pterygomaxillary fissure are measured for all children every two years, namely at age 8,10,12 and 14, and research interest is to investigate the how the age and gender affect the distance. The data has been studied by Potthoff and Roy [1964], Pinheiro and Bates [2000] and Georges Monette [2010]. Specifically, in George's study, it demonstrates that a linear regression model with only fixed effects cannot explain the data variation well, and a linear mixed model is recommended.

First, we take a comprehensive overview of the distance over years regarding each individual, where the results are summarized in figure 6.1. In this figure, "M" stands for male and F stands for "female", and follow by every child's index. At first glance, there seems to be a distinct difference between the males' pattern and females' pattern, and variation exists among subjects. To further discuss the difference between genders, we plot the mean responses for males and females respectively, which is shown in figure 6.2.

By observing its overall pattern, we can see that the distance has a positive association with age regardless of genders; Secondly, the mean distance of males stays at a high level compared to females; In addition, since the increasing trend of males is steeper, the variation of distance over time for males is more distinct than females. Following the first two results, it is plausible to believe the main effects of age and gender are significant. With the third result, we may believe 


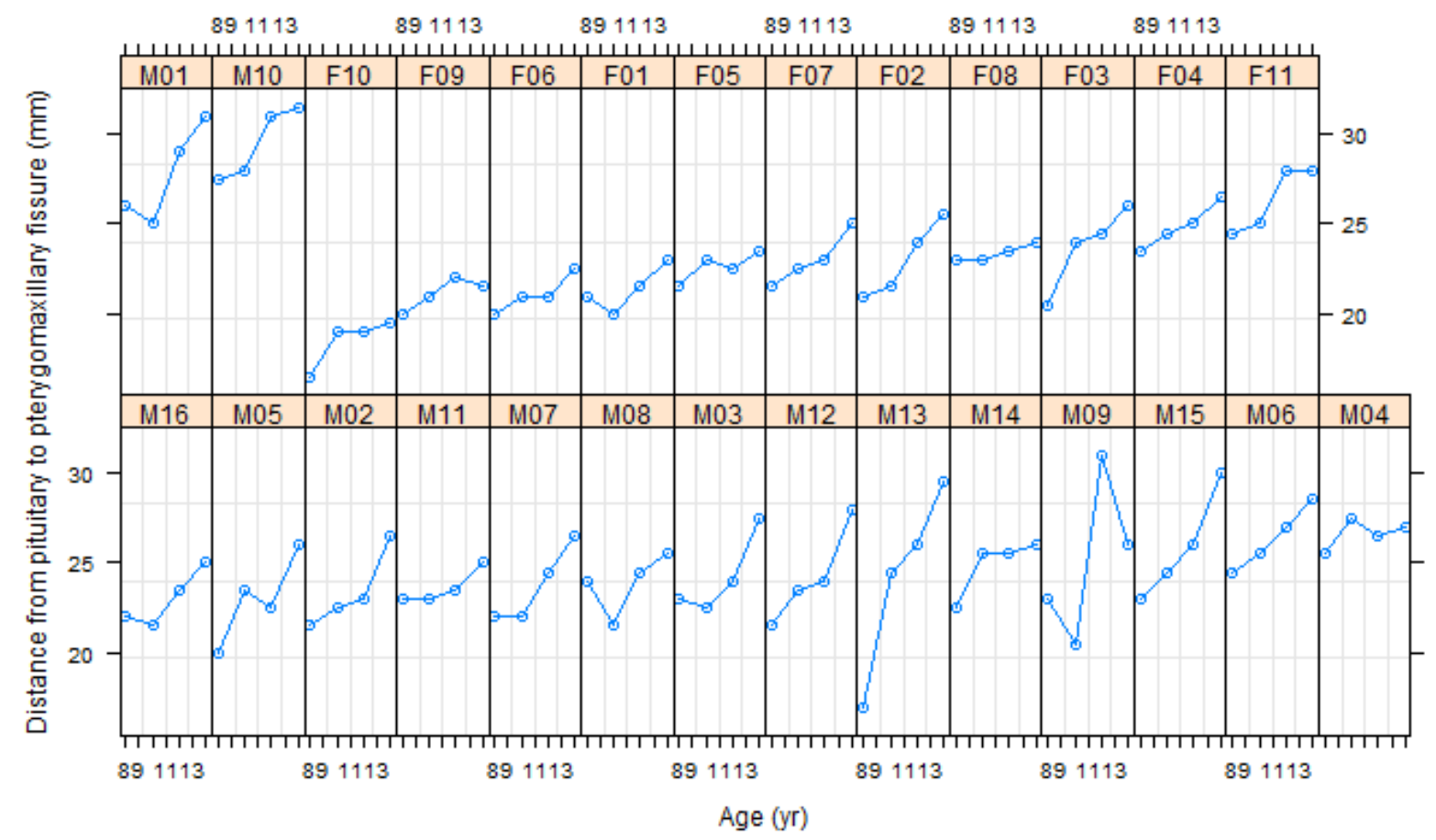

Figure 6.1: Distance of every child.

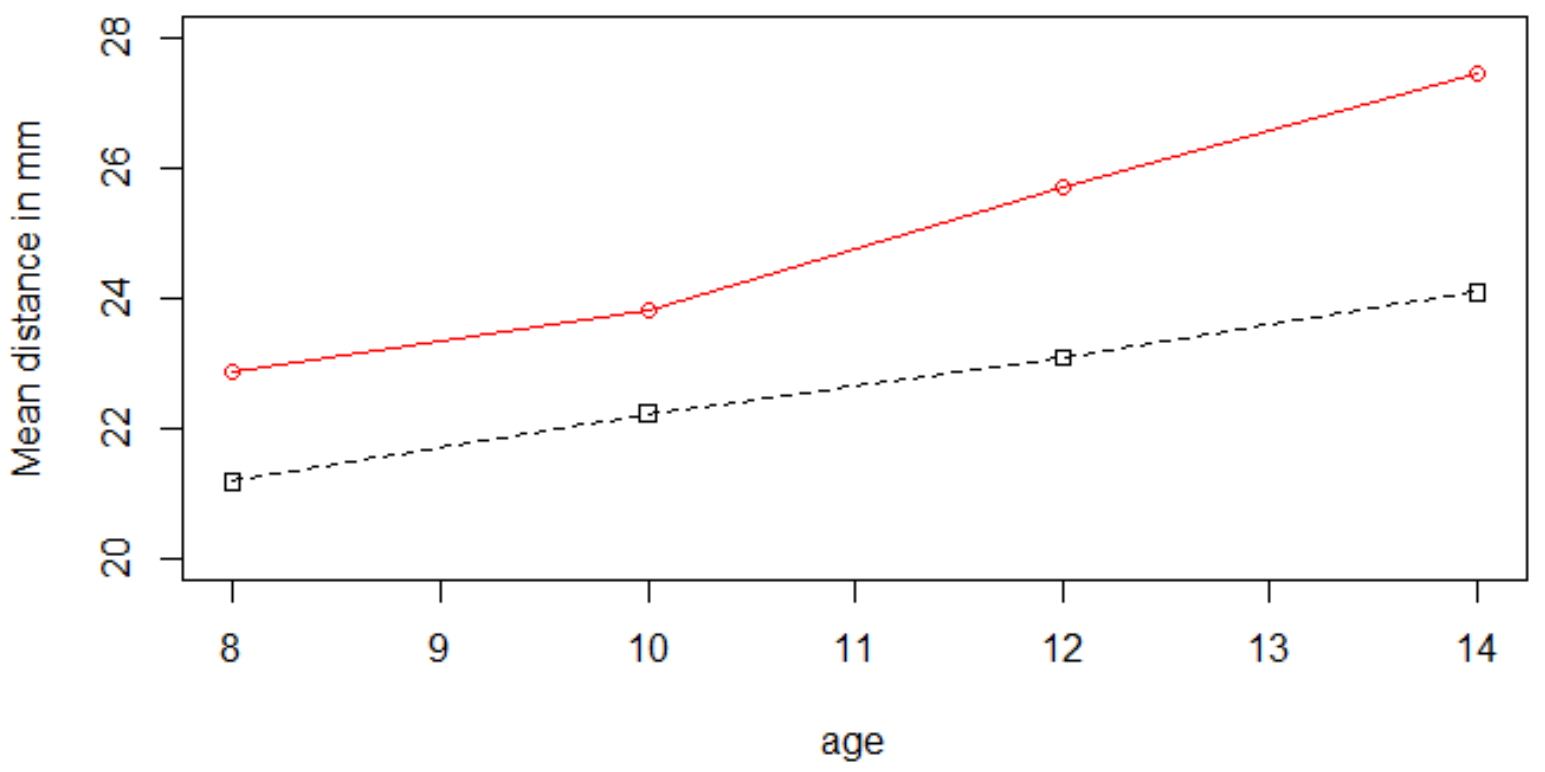

Figure 6.2: Mean distance, red for males and black for females. 
that the interaction of age $*$ gender is significant.

When the data is fitted with a fixed effect model, some results from Georges Monette [2010] study are presented as follows:

Table 6.1: Georges' Study 1

\begin{tabular}{lllll}
\hline Coefficients & Estimate & std.error & t-value & P-value \\
\hline Intercept & 16.3406 & 1.4162 & 11.538 & $e^{-16}$ \\
\hline age & 0.7844 & 0.1262 & 6.217 & $1.07 e^{-08}$ \\
\hline female & 1.0321 & 2.2188 & 0.465 & 0.643 \\
\hline age*female & -0.3048 & 0.1977 & -1.542 & 0.126 \\
\hline
\end{tabular}

Since "male" is set as the baseline, it does not exist in Table 6.1. For the variable age, it is treated as a numerical value. From this table, with a regular $5 \%$ significance level, we can see that both of "female" and "age*female" are insignificant, and that indicates both of them could be dropped. However, when an F-test is conducted for gender, the result is given as

Table 6.2: Georges' Study 2

\begin{tabular}{lllll}
\hline s & DF & denDF & F-value & P-value \\
\hline Sex & 2 & 104 & 14.97688 & $<0.00001$ \\
\hline
\end{tabular}

From Table 6.2 we may conclude that "female" is a significant effect, which contradicts to the earlier analysis in Table 6.1. Therefore, a linear regression model can not exploit the consistency, and some other techniques are required. 


\subsection{Analysis in Linear Mixed Models}

When the data is standardized, a recommended linear mixed model in George's study is given by

$$
\text { distance }_{i j}=\beta_{1 j} \times \text { age }+\beta_{2 j} \times \text { gender }+\beta_{3 j} \times \text { age } * \text { gender }+b_{1 j}+b_{2 j} \times \text { age }+\epsilon_{i j},
$$

where $\beta_{i j}{ }^{\prime} s$ are coefficients for fixed effects and $b_{i j}{ }^{\prime} s$ are coefficients for random effects. Moreover, the autoregressive process of order 1 is considered to be the structure of random effects covariance. Note that there is no intercept for fixed effects in this model since data is standardized. However, there is an intercept for random effect, since variation exists among individuals. The corresponding AIC and BIC are presented in Table 6.1.

Table 6.3: Marginal criteria for model (6.1)

\begin{tabular}{lll}
\hline Model & mAIC & mBIC \\
\hline$(6.1)$ & 446.8076 & 470.6072 \\
\hline
\end{tabular}

Next, considering a more complicated structure, we add a quadratic term age ${ }^{2}$ for both fixed and random effects. Therefore, the model is given by

$$
\begin{aligned}
\text { distance }_{i j}= & \beta_{1 j} \times \text { age }+\beta_{2 j} \times \text { gender }+\beta_{3 j} \times \text { age } * \text { gender }+\beta_{4 j} \times \text { age }^{2}+b_{1 j} \\
& +b_{2 j} \times \text { age }+b_{3 j} \times \text { age }^{2}+\epsilon_{i j} .
\end{aligned}
$$

Regarding model (6.2), the covariance of random effects is arbitrary. By implementing the standard "Imer" function in R, we can obtain the full model. Note that we do not drop any variable even its p-value is smaller than 0.05 . To check whether this model is too complex, we may implement adaptive lasso via cross-validation to detect the possibility of further simplifying it. 


\subsection{Implementation and Results}

We randomly draw 14 subjects as the training data, while the rest 13 subjects form the test data. To convert the data into a matrix form, we code "female" $=1$ and "male" $=0$. Since subgroup size is 4 , then for the training data, fixed design matrix is 56 by 4 and random design matrix is 56 by 3 , and the distance is observed 56 times. Choosing $30 \lambda^{\prime} s$ uniformly distributed from 0.1 to 3 , and implementing adaptive lasso penalty function, we obtain 20 candidate models (actually most candidate models have the same form). Afterwards, fitting the candidate models with the test data, where fixed design matrix is 52 by 4 and random design matrix is 52 by 3, we select the model with smallest mBIC. The result shows that age ${ }^{2}$ is dropped for fixed effects and (age, age $\left.{ }^{2}\right)$ are dropped for random effects. As a result, the simplified model takes the form:

$$
\text { distance }_{i j}=\beta_{1 j} \times \text { age }+\beta_{2 j} \times \text { gender }+\beta_{3 j} \times \text { age } * \text { gender }+b_{1 j}+\epsilon_{i j},
$$

where we call it model (6.3).

To compare the three models, we summarized the criterion corresponding to each model in Table 6.4, where the model (6.1) is the one suggested by Georges Monette [2010], model (6.2) is the manipulated complex one and model (6.3) is the simplified model selected by adaptive lasso via cross-validation.

Table 6.4: Marginal criteria for all models

\begin{tabular}{lll}
\hline Model & mAIC & mBIC \\
\hline$(6.1)$ & 446.8076 & 470.6072 \\
\hline$(6.2)$ & 448.5817 & 470.0387 \\
\hline$(6.3)$ & 445.7572 & 461.85 \\
\hline
\end{tabular}

In this table, we can see that mAIC of model (6.3) is slightly smaller than the other two models, 
while its mBIC is distinctly smaller than other two models. In general, it possesses the smallest mAIC and mBIC simultaneously, which indicates that model (6.3) is the best among all. Moreover, the sample size in this application is only 27 , which indicates the approach does perform very well for a moderately small sample.

Form this real data example, we can tell that our algorithm is very likely to select a good model in reality, which is compatible with our earlier analysis. 


\section{CHAPTER 7 CONCLUSION AND FURTHER DISCUSSION}

In the dissertation, we mainly propose a penalized method to achieve model selection in linear mixed models, which is called adaptive lasso via cross-validation. In this chapter, we will make a brief summary of the construction of the penalized function and the implementation of this algorithm. In addition, the advantages and limitations are analyzed, and some possible ways to improve the approach are also discussed at the end.

\subsection{Conclusion}

The proposed approach follows the general idea of shrinkage methods, starting with assigning several tuning parameters for a penalized likelihood function to continuously shrink some coefficients to zero. Nevertheless, because of the complex structure of mixed models, it is often dealing with a complicated function including too many parameters. To simplify the model, a modified Cholesky decomposition is employed to reparameterize the model, which facilitates the construction of penalized function. More specifically, only the diagonal elements of the decomposed matrix are considered in the penalty term, which significantly simplifies the functional complexity.

Since each tuning parameter determines a candidate simplified model, and our objective is to select the "best" one among all. Therefore, we divide the data into the training set and test set, while constructing a suite of models regarding training set and evaluating the candidate models in the test set. Afterwards, the tuning parameter corresponding to the best model for test set is selected. Numerous examples of simulation studies indicate that selection accuracy is improved with cross-validation. After obtaining the tuning parameter with smallest mBIC for the validation data, we screen the fixed effects to even improve the performance.

In addition to real-life examples and empirical analysis, asymptotic results are provided to ensure the validity from the theoretical perspective. It demonstrates that the procedure almost always identifies the true sparse pattern when the sample size is large enough. Moreover, all the 
coefficient estimates converge to the true parameters simultaneously in probability.

In terms of computation, the original penalized function takes the form of an integral, therefore EM-algorithm is implemented to solve the minimum iteratively. However, the expectation in the E-step under current estimates is not a regular function, thus some algebra transformations are made to write the target function as a penalized quadratic term with respect to fixed and diagonal parameters and a regular quadratic term for non-diagonal effects. Considering the convexity of the penalized quadratic term, coordinate descent algorithm is employed to attain the minimum for the penalized quadratic term. Meanwhile, the minimum of the regular quadratic term can be easily attained.

Finally, some limitations are also discussed in the dissertation. For example, the method may perform poorly in the high dimensional case. In addition, the selection results become worse as sample size decreases, consequently, the procedure may be inefficient for a moderately small sample. Moreover, all the results are derived under normal assumptions. As a result, the approach may fail when normal assumptions are not met.

Nevertheless, in spite of those limitations, adaptive lasso via cross-validation is still a very productive method in linear mixed model selection in general, which performs quite well in most cases.

\subsection{Further Discussion}

As mentioned in the limitation, our approach relies on normal assumptions. When the normal assumptions are not met, a natural way is to transform the variable to make the normal assumptions satisfied.

Secondly, it has been mentioned that the most reliable inferences for mixed models are done with Markov Chain Monte Carlo (MCMC) or parametric bootstrap tests (Social Science) to screen fixed effects. However, considering the cost of computation, we apply likelihood ratio test instead. To even improve the selection accuracy, we may consider conducting a more accurate test if longer run times are allowed. 
Moreover, we observe that corrected selection rates follow a negative trend when sample size or subgroup size decreases. According to this result, a natural conjecture is that the approach performs poorly for a moderately small sample. To facilitate its application in small samples, we may either consider parametric or non-parameter bootstrap to generate a pseudo sample with a larger size. 


\section{BIBLIOGRAPHY}

Hirotugu Akaike. Information theory and an extension of the maximum likelihood principle. International Symposium on Information Theory, pages 267-281, 1973.

E.J. Bedrick and C.L. Tsai. Model selection for multivariate regression in small samples. Biometrics, 50:226-231, 1994.

A. Bondell, H. D.and Krishna and S. K. Ghosh. Joint variable selection for fixed and random effects in linear mixed-effects models. Biometrics, 66:1069-1077, 2010.

L. Breiman. Bagging predictors. Machine Learning, 26(2):123-140, 1996.

Emmanuel Candes and Terence Tao. The dantzig selector: Statistical estimation when $\mathrm{p}$ is much larger than n. The Annals of Statistics, 35(6):2313-2351, 2007.

Z. Chen and D. B Dunson. Random effects selection in linear mixed models. Biometrics, 59: 762-769, 2003.

Andr-Louis Cholesky. Cholesky decomposition. Cholesky’s 1910 manuscript, 1910.

A.P. Dempster, N.M. Laird, and D.B. Rubin. Maximum likelihood from incomplete data via the em algorithm. Journal of the Royal Statistical Society, 39:1-38, 1977.

D. Donoho and M. Elad. Optimally sparse representation in general (nonorthogonal) dictionaries via 11-norm minimizations. Proceedings of the National Academy of Science USA, 1005:2197$2202,2002$.

B.Hastie Efron, Johnstone I. T., and R. Tibshirani. Least angle regression. The Annals of Statistics, 32:407-499, 2004.

J Fan and J Lv. Variable selection via nonconcave penalized likelihood and oracle properties. Journal of the American Statistical Association, 20:101-148, 2001. 
S. D. Foster, A. P. Verbyla, and W. S. Pitchford. Incorporating lasso effects into a mixed model for quantitative trait loci detection. Agric. Biol. Environ. Stat, 12:300-314, 2007.

J. Friedman, T. Hastie, H. Hoefling, and R. Tibshirani. Pathwise coordinate optimization. Annals of Applied Statistics, 7(3):302-332, 2007.

Jerome Friedman, Trevor Hastie, and Rob Tibshirani. Regularization paths for generalized linear models via coordinate descent. 2009.

Jim Frost. The danger of overfitting regression models. The Minitab Blog, 2015.

Georges Monette. Longitudinal Data Analysis with Mixed Models, 2010. URL http://wiki. math.yorku.ca/SPIDA_2010

S. Greven and T. Kneib. On the behaviour of marginal and conditional aic in linear mixed models. Biometrika, 97:773-789, 2010.

Robert Grossman, Giovanni Seni, John. Elder, Nitin. Agarwal, and Huan Liu. Improving accuracy through combining predictions. Ensemble Methods in Data Mining, 2010.

E. J. Hannan and B. G. Quinn. The determination of the order of an autoregression. Journal of the Royal Statistical Society, 41:190-195, 1979.

C. R Henderson. Estimation of genetic parameters. Ann. Math. Statist, 21:309-310, 1950.

C. R. Henderson, Oscar Kempthorne, S. R. Searle, and C. M. von Krosigk. The estimation of environmental and genetic trends from records subject to culling. Biometrics. International Biometric Society, 15(2):192-218, 1959.

C. Hurvich and C. Tsai. Regression and time series model selection in small samples. Biometrika, 76:297-307, 1989.

C. Hurvich and C. Tsai. A corrected akaike information criterion for vector autoregressive model selection. Journal of Time Series Analysis, 14:271-279, 1993. 
C. Hurvich and C. Tsai. Model selection for extended quasi-likelihood models in small samples. Biometrics, 51:1077-1084, 1995.

C. Hurvich, R. Shumway, and C. Tsai. Improved estimators of kullback-leibler information for autoregressive model selection in small samples. Biometrika, 77:709-719, 1990.

C. M. Hurvich and C. L. Tsai. The impact of model selection on inference in linear regression. American Statistician, 44:214-217, 1990.

J. G. Ibrahim, H. Zhu, Garcia. R. I., and R. Guo. Fixed and random effects selection in mixed effects models. Biometrics, 67:495-503, 2011.

Ron. Kohavi. A study of cross-validation and bootstrap for accuracy estimation and model selection. Proceedings of the Fourteenth International Joint Conference on Artificial Intelligence, 2 (12):1137-1143, 1995.

C. L. Mallows. Some comments on cp. Technometrics, 15:661-675, 1973.

N. Meinshausen and P Bhlmann. Variable selection and high dimensional graphs with the lasso. technical report, 2004. doi: 10.1145/219717.219748.

T. M. Mitchell. The need for biases in learning generalizations. CBM-TR, New Brunswick, New Jersey, USA, 1980.

Samuel Muller, J. L. Scealy, and A. H. Welsh. Model selection in linear mixed models. Statistical Science, 28(2):135-167, 2013.

Xiao Ni, Hao Helen Zhang, and Daowen Zhang. Automatic model selection for partially linear models. Journal of Multivariate Analysis, 100:2100-2111, 2010.

H. Peng and Y. Lu. Model selection in linear mixed effect models. Multivariate Annal, 109: 109-129, 2012.

J. C. Pinheiro and D. M. Bates. Model Selection in Linear Mixed Models. New York, 2000. 
R. F. Potthoff and S. N. Roy. A generalized multivariate analysis of variance model useful especially for growth curve problems. Biometrika, 51:313-326, 1964.

A. C. Rencher and F. C. Pun. Inflation of $\mathrm{r}$ in best subset regression. Technometrics, 22:49-54, 1980.

J. Schelldorfer, P. Bhlmann, and S. van de Geer. Estimation for high-dimensional linear mixedeffects models using 11-penalization. Scandinavian Journal of Statistics, 38:197-214, 2011.

Jeff Schneider. Judging model quality by residuals. 1997.

G. Schwarz. Estimating the dimension of a model. The Annals of Statistics, 6:461-464, 1978.

Jun Shao. Linear model selection by cross-validation. American Statistical Association, 88(422): 39-41, 61993.

Jun Shao. An asymptotic theory for linear model selection. Statistica Sinica, 7:221-264, 1997.

M. Stone. The generalized weierstrass approximation theorem. Mathematics Magazine, 21(4): 167-184, 1948. doi: 10.1145/219717.219748.

M. Stone. An asymptotic equivalence of choice of model by cross-validation and akaike's criterion. Journal of the Royal Statistical Society, 39(1):44-47, 1977.

N. Sugiura. Further analysis of the data by akaikes information criterion and the finite corrections. Comm. Statist, 7:13-26, 1978. doi: 10.1145/219717.219748.

R. Tibshirani. Regression shrinkage and selection via the lasso. Journal of the Royal Statistical Society, 58:268-288, 1996.

F. Vaida and S. Blanchard. Conditional akaike information for mixed-effects models. Biometrika, $92: 351-370,2005$.

Larry Wasserman and Kathryn Roeder. High-dimensional variable selection. The Annals of Statistics, 37(5):2178-2201, 2009. 
Tong Wu and Kenneth Lange. Coordinate descent algorithms for lasso penalized regression. The Annals of Applied Statistics, 2(1):224-244, 2008.

Jingwei Xiong and Junfeng Shang. A cross-validation method for linear regression model selection. The 2016 International Conference on Data Mining, 2016. doi: 10.1145/219717.219748.

Cun-Hui Zhang. Nearly unbiased variable selection under minimax concave penalty. he Annals of Statistics, 38(2):894-942, 2010.

H. Zou. The adaptive lasso and its oracle properties. Journal of the American Statistical Association, 101:1418-1429, 2006. 


\section{APPENDIX A SELECTED R PROGRAMS}

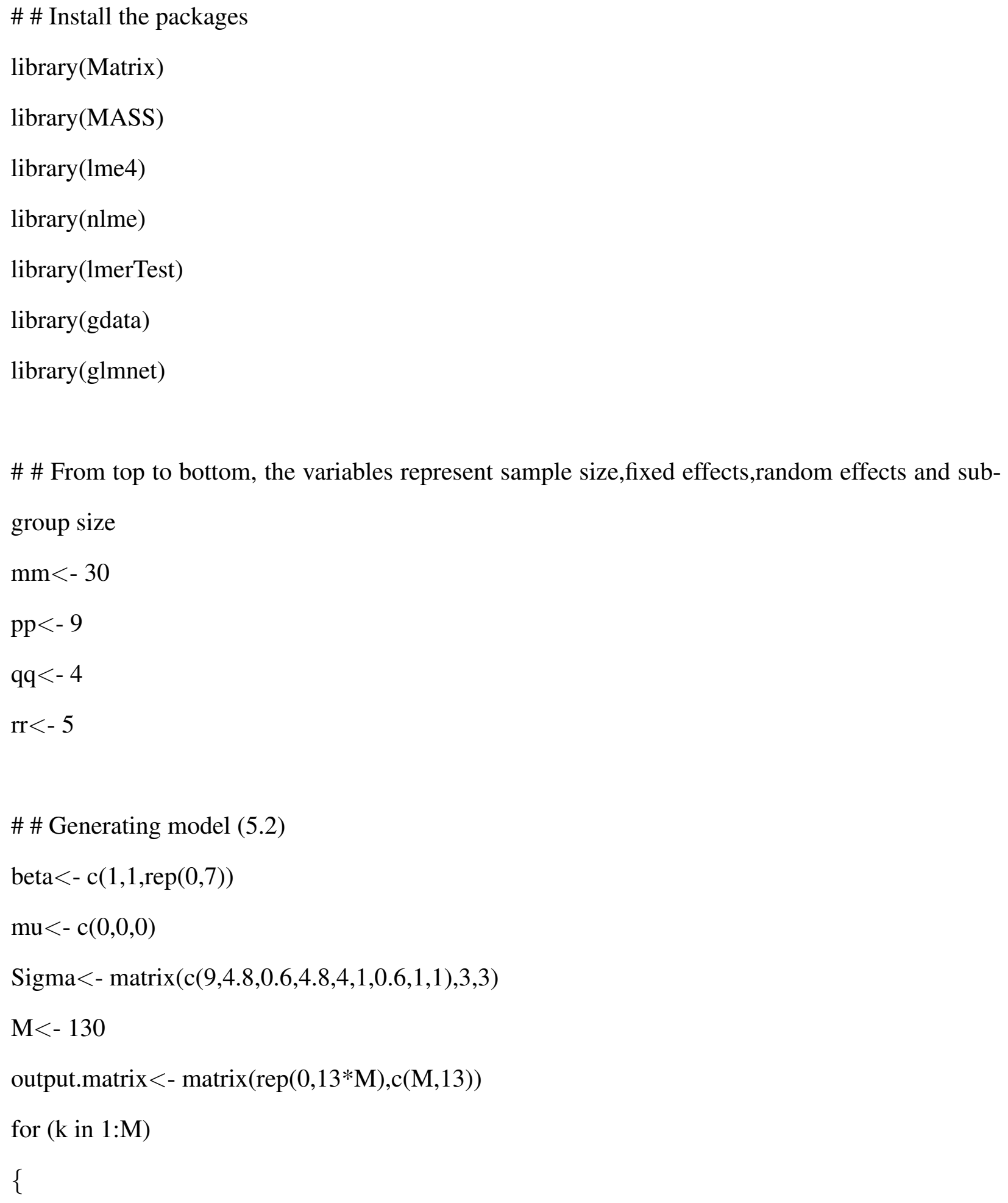









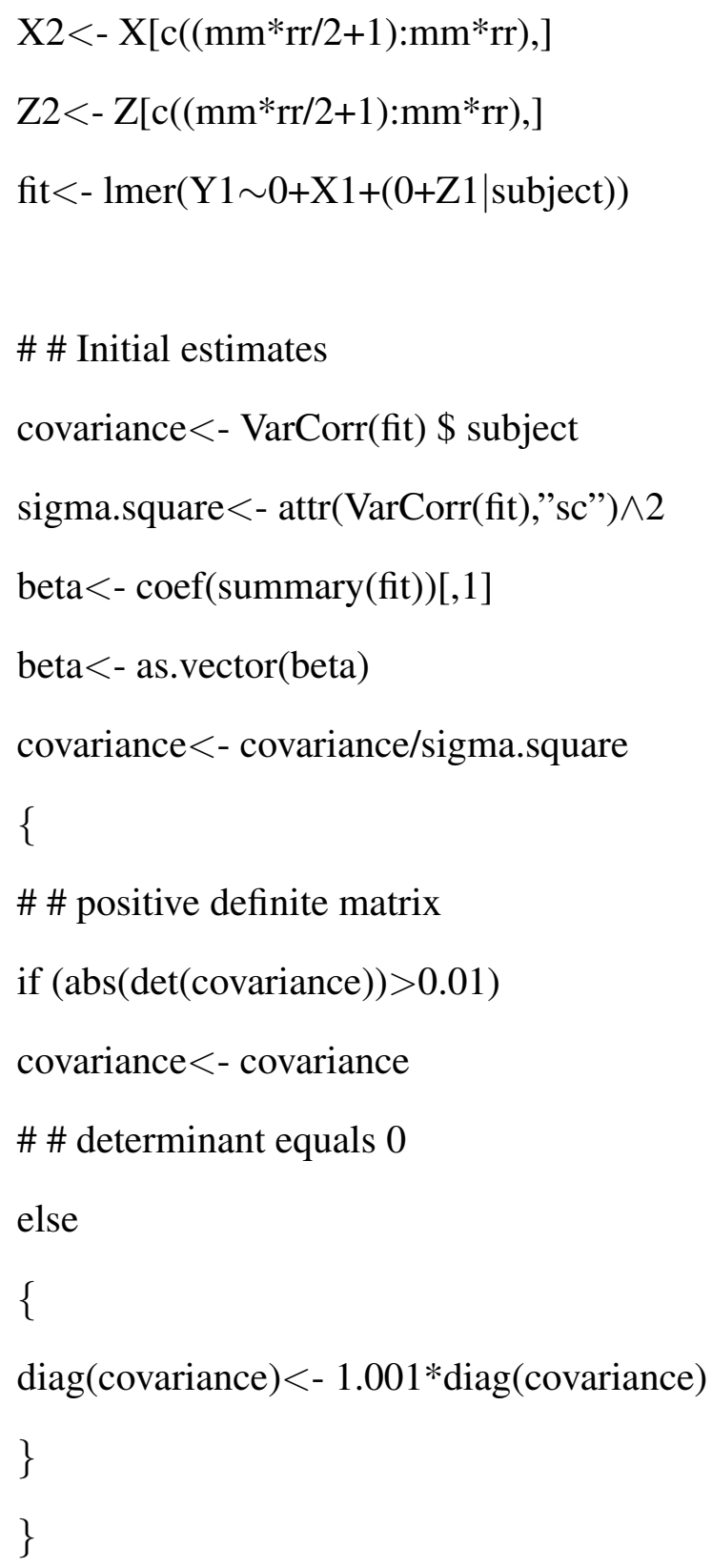




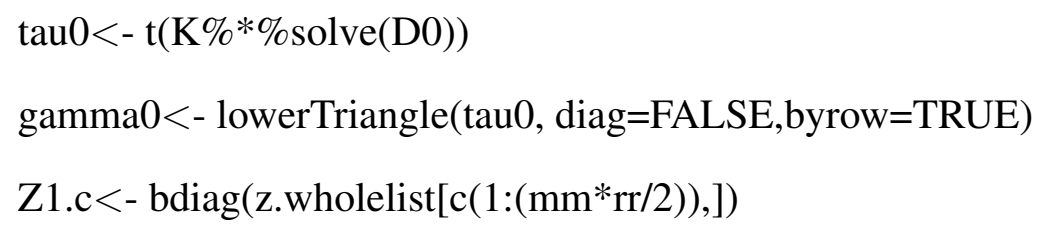




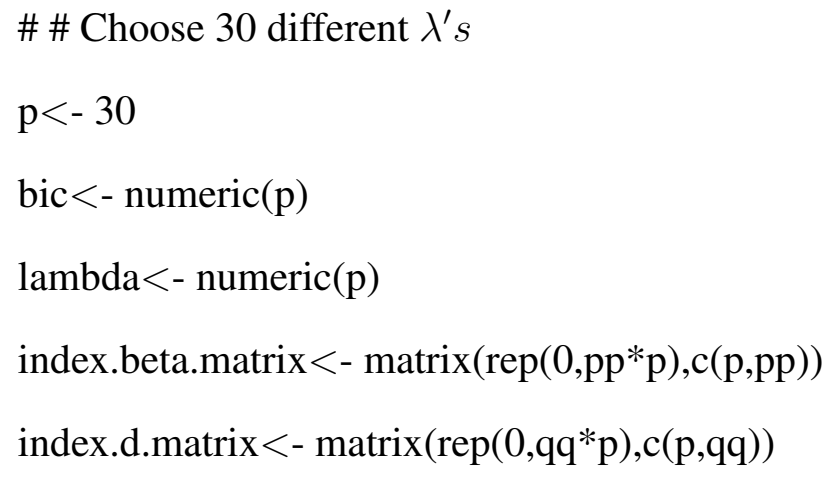


U.w0<- sigmasq.w0* solve(t(tau.tuta0)\%*\%D.tuta0\%*\%t(Z1.c)\%*\%(Z1.c)\%*\%D.tuta0\%*\% tau.tuta0+diag $(\mathrm{qq} * \mathrm{~mm} / 2))$

G.w0<- U.w0+b.w0\%*\%t(b.w0)

\#\#Write out the middle matrix

\#\# split into block matrix

first $<-\mathrm{t}(\mathrm{X} 1) \% * \% \mathrm{X} 1$

second <- as.matrix(t(X1)\%*\%Z1.c\%*\%diag(as.numeric(tau.tuta0\%*\%b.w0))\%*\%mIq)

third $<-\mathrm{t}$ (second)

fourth $<-$ as.matrix $(\mathrm{t}(\mathrm{mIq}) \% * \%((\mathrm{t}(\mathrm{Z} 1 . \mathrm{c}) \% * \% \mathrm{Z} 1 . \mathrm{c}) *(\operatorname{tau} . \operatorname{tuta} 0 \% * \% \mathrm{G} . \mathrm{w} 0 \% * \% \mathrm{t}(\mathrm{tau} . \mathrm{tuta} 0))) \% * \%$ (mIq))

\#\#Combine the above matrices to make the whole matrix

Middle.matrix <- rbind(cbind(first,second), cbind(third,fourth))

Middle2.matrix <- cbind(X1,as.matrix(Z1.c\%*\%diag(as.numeric(tau.tuta0\%*\%b.w0))\%*\%mIq))

\#\#Build the function for beta and d, named beta.d.

$\mathrm{f}<-$ function(beta.d) $\{\mathrm{t}($ beta.d) $\% * \%$ Middle.matrix $\% * \%$ beta.d- $2 * \mathrm{t}(\mathrm{Y}) \% * \%$ Middle2.matrix $\% * \%$ beta.d+lambda[j]*sum(abs(beta.d)*abs(1/c(beta,d)))

\}

fit1<- optim(rep(0, (pp+qq)), f, NULL, method = "L-BFGS-B", lower = rep $(-100,(p p+q q))$, upper $=\operatorname{rep}(100,(p p+q q))$, control=list $(\operatorname{maxit}=10000))$

beta.w $1<-$ fit 1 \$par[1:pp] 
$\mathrm{d} 1<-$ fit $1 \$ p a r[(p p+1):(p p+q q)]$

\#\#beta.d update completes

\#\#Next

bvector0<- as.numeric(b.w0[-seq(qq,qq*mm/2,qq),])

indexb.w0<- rep(sequence $(\mathrm{c}(1:(\mathrm{qq}-1))), \mathrm{mm} / 2)+(\mathrm{qq}-1) * \mathrm{rep}(0:(\mathrm{mm} / 2-1)$, each $=(\mathrm{qq}-1) * \mathrm{qq} / 2)$

b0quan.mean $<-$ bvector0[indexb.w0]

modifyU.w0<- U.w0[-qq*c(1:mm/2),-qq*c(1:mm/2)]

b0quan.variance <- modifyU.w0[indexb.w0,indexb.w0]

E.b0quan.sq $<-$ b0quan.variance+b0quan.mean\%*\%t(b0quan.mean)

Dquan 1<- bdiag(replicate(mm/2,list(d1[rep(c(2:qq), c(1:(qq-1)))]\%*\%

$\mathrm{t}(\mathrm{d} 1[\operatorname{rep}(\mathrm{c}(2: \mathrm{qq}), \mathrm{c}(1:(\mathrm{qq}-1)))]))))$

modifyZ $<-$ Z.c [,-seq(1,(mm/2*rr-3),qq)]

index $Z<-\operatorname{rep}(\mathrm{c}(1,2,2,3,3,3), \mathrm{mm} / 2)+3 * \operatorname{rep}(0:(\mathrm{mm} / 2-1)$, each=6)

Zquan $<$ - modifyZ[,indexZ]

Zquansq $<-\mathrm{t}($ Zquan $) \% * \%$ Zquan

B.list $<-$ split(b.w0,ceiling(seq_along(b.w0)/4))

Bquan $<-$ as.matrix(bdiag(lapply(B.list,buildin.new)))

\#\#\#\#\#Continue to complete the middle term

Zsan $<-($ Zquan $) \% * \% Z 1 . c$

bsan $<-$ b0quan.mean\%*\%t(b.w0)

dsan<- mapply(cbind,replicate(qq,list(d1[rep(c(2:qq),c(1:(qq-1)))])))

Dsan $<-\operatorname{bdiag}(\operatorname{replicate}(\mathrm{mm} / 2, \operatorname{list}(\operatorname{dsan})))$ 
dvector $<-$ mapply $($ c,replicate $(\mathrm{mm} / 2, \mathrm{~d} 1))$

\#\# The function to be optimized for gamma

$\mathrm{g}<-$ function(gamma) \{

as.numeric(t(gamma)\%*\%t(Iquan)\%*\%

(as.matrix(Eb0quan.sq*Dquan $1 *$ Zquansq))\%*\%Iquan\%*\%gamma+

$2 * \mathrm{t}($ gamma $) \% * \% \mathrm{t}($ Iquan $) \% * \%($ as.matrix $(Z s a n * b s a n * D s a n)) \% * \%$ dvector-

$2 * \mathrm{t}($ gamma $) \% * \% \mathrm{t}($ Iquan $) \% * \% \mathrm{t}($ Bquan $) \% * \%$

bdiag(replicate(mm,list(diag(d1))))\%*\% t(Z1.c)\%*\%(Y1-X1\%*\%beta.w1))

\}

fit2<- optim(c(1:(qq*(qq-1)/2)), g, NULL, method = "L-BFGS-B", lower = rep(-100, $\left(\mathrm{qq}^{*}(\mathrm{qq}-\right.$ 1)/2)), upper $=\operatorname{rep}\left(100,\left(q q^{*}(q q-1) / 2\right)\right)$, control=list $\left.(\operatorname{maxit}=10000)\right)$

gamma $1<-$ fit $2 \$$ par

result[i,]<- c(beta.w1,d1,gamma1)

$\mathrm{i}<-\mathrm{i}+1$

if $(\operatorname{sum}(\operatorname{abs}(\mathrm{c}($ beta.w1,d1, gamma1)-c(beta.w0,d0, gamma0)) $)<$ tol $)$

break

gamma $0<-$ gamma1

$\mathrm{d} 0<-\mathrm{d} 1$

beta.w $0<-$ beta.w 1

\}

vectorbeta $<-\operatorname{rep}(0, p p)$

vectord $<-\operatorname{rep}(0, q q)$ 


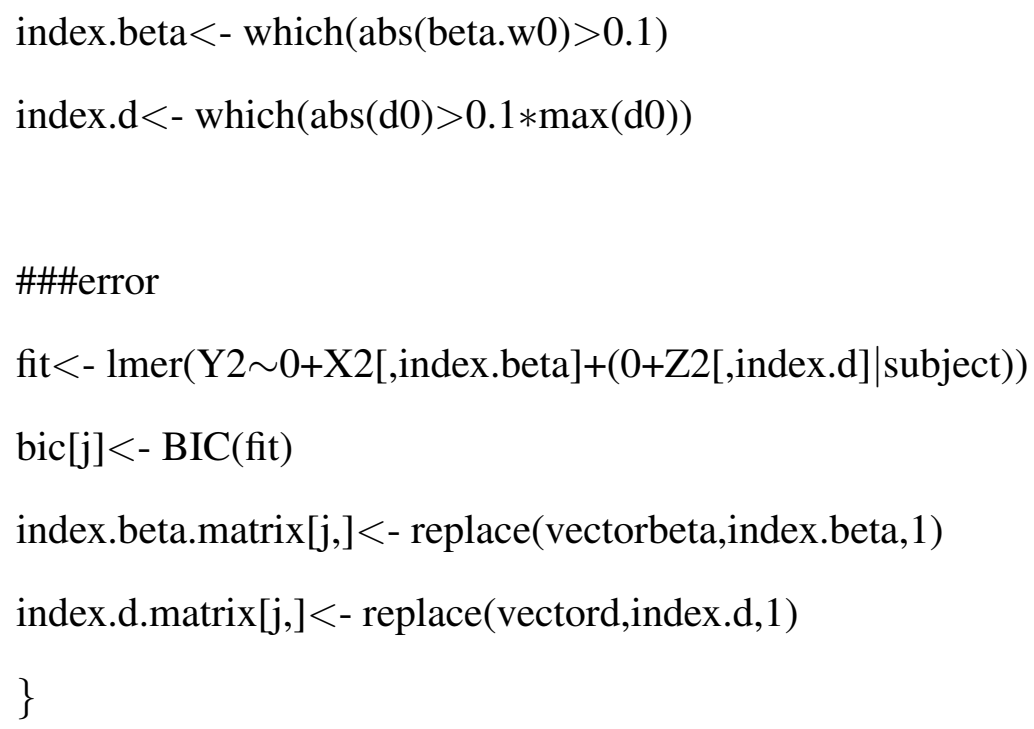


output.matrix 